\title{
MACHINE TO MACHINE OVERLAY NETWORK OVER RANDOM ACCESS CHANNEL OF LTE FOR SMART CITY
}

by

\author{
Nargis Khan \\ M.Sc. in Computer Science, Ryerson University, Toronto, Canada, 2011 \\ B.Sc. in Computer Science \& Engineering, Jahangirnagar University \\ Dhaka, Bangladesh, 2004 \\ A dissertation \\ presented to Ryerson University \\ in partial fulfillment of the \\ requirements for the degree of \\ Doctor of Philosophy \\ in the Program of \\ Computer Science
}

Toronto, Ontario, Canada, 2018

(C)Nargis Khan 2018 



\section{AUTHOR'S DECLARATION FOR ELECTRONIC SUBMISSION OF A DISSERTATION}

I hereby declare that I am the sole author of this dissertation. This is a true copy of the dissertation, including any required final revisions, as accepted by my examiners.

I authorize Ryerson University to lend this dissertation to other institutions or individuals for the purpose of scholarly research.

I further authorize Ryerson University to reproduce this dissertation by photocopying or by other means, in total or in part, at the request of other institutions or individuals for the purpose of scholarly research.

I understand that my dissertation may be made electronically available to the public. 



\begin{abstract}
MACHINE TO MACHINE OVERLAY NETWORK OVER RANDOM ACCESS CHANNEL OF LTE FOR SMART CITY

Doctor of Philosophy, 2018

Nargis Khan

Computer Science

Ryerson University
\end{abstract}

The purpose of this dissertation work is to investigate the perspective of the application areas of machine to machine (M2M) communications specifically to build smart city. To have ubiquitous coverage over the city, we consider cellular infrastructure. We propose a M2M overlay network over the physical random access channel (PRACH) of LTE. Based on this architecture, we conduct a case study in a vehicular context and proposed a non-priority CSMA/CA based vehicular M2M (VM2M) overlay networks. The overlay architecture is implemented using a dedicated subset of preambles at the physical layer, and a carrier sense multiple access with collision avoidance (CSMA/CA) mechanism similar to the one used in IEEE 802.15.4 at the medium access control (MAC) layer. We evaluate the performance and interaction of regular human to human $(\mathrm{H} 2 \mathrm{H})$ traffic and VM2M traffic, in particular, the impact of $\mathrm{RACH}$ resource configuration and preamble format $(\mathrm{PF})$ in large cells. We have shown that the proposed LTE based VM2M architecture is better suited for smart city scenarios with higher vehicular speed and larger distances without sacrificing performance of $\mathrm{H} 2 \mathrm{H}$ traffic.

To transmit priority messages with high data rate and more reliably, we also propose a novel priority based CSMA/CA machine to machine (PM2M) overlay network over LTE. The PM2M overlay“s MAC is more sophisticated with priorities. The architecture is same 
in the physical layer with dedicated preambles and in MAC layer priority based CSMA/CA is used, which is similar to the one of IEEE 802.15.6.

Finally, We propose an analytical model to evaluate the impact of error in the MAC layer of overlay networks during sensing the medium. We develop a 3 dimensional Discrete Time Markov chain (DTMCs) in order to model the backoff procedure of CSMA/CA mechanism with backoff error. We investigated the capacity of PM2M networks for with and without considering backoff error. We have shown that overlay network allows fair coexistence of PM2M and $\mathrm{H} 2 \mathrm{H}$ traffic. To validate our scheme we compare the proposed approach with a reference approach. By performance evaluation, we have shown that PM2M overlay out performs compared with reference approach. 


\section{Acknowledgements}

There are many people to whom I like to express my gratitude. Without their support and encouragement it would not be possible to finish this work.

First and foremost, I am very thankful to my supervisor, Dr. Jelena Mišić for accepting me as her $\mathrm{PhD}$ student. Her continual support and guidance helped me to complete this work. I was greatly inspired by her excitement, vision, and creativity. She has been abundantly helpful and have assisted me in numerous ways. Without her continual support and thoughtful mentoring, many of the ideas in this thesis would never have come to fruition.

I would also like to appreciate my thesis committee, Dr. Isaac Woungang, Dr. Vojislav B. Mišić, Dr. Alagan Anpalagan from Ryerson University and Dr. Nidal Nasser from Alfaisal University for taking the time and making the effort to review this thesis and provide me with their insightful comments.

I am very grateful to my parents for their endless love and support.

Finally, I dedicate this thesis to my parents, my husband Dr. Lutful Karim, my daughter Naziat Karim and my son Sahat Karim, who supported me unconditionally and motivated and inspired me and made my time more enjoyable during these years. 

To my beloved parents, sister, brothers, husband, daughter and son for their support and encouragement 



\section{Contents}

Author's Declaration $\ldots \ldots \ldots \ldots \ldots \ldots \ldots$ iii

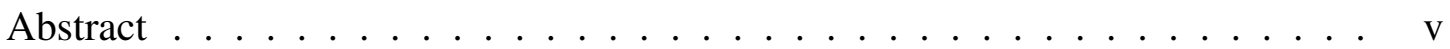

Acknowledgements ................... vii

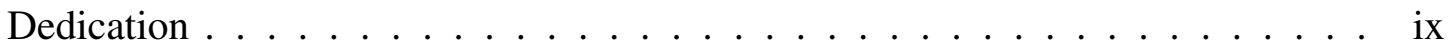

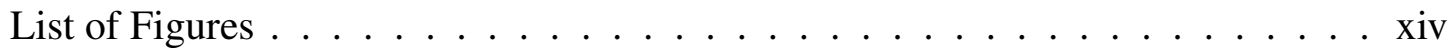

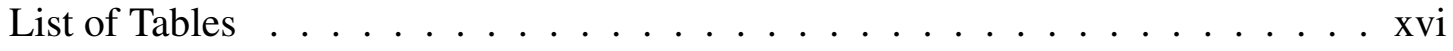

1 Introduction 1

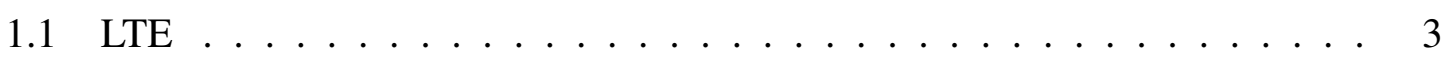

1.1.1 Random access procedure $\ldots \ldots \ldots \ldots \ldots \ldots$

1.1.2 RACH overload control approaches . . . . . . . . . . . 5

1.1.3 Frame structure, configuration and PRACH resources in LTE . . . . 7

1.1.4 Preambles formats . . . . . . . . . . . . . . 8

1.2 Vehicular Adhoc network (VANET) _ . . . . . . . . . . . . 10

1.2.1 Limitations of IEEE 802.11p and features of LTE . . . . . . . 10

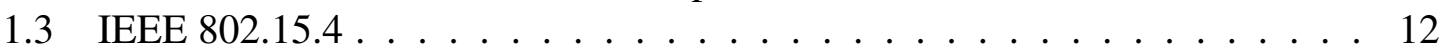

1.3.1 MAC channel access mechanism . . . . . . . . . . . . 13

CSMA/CA medium access control mechanism . . . . . . . . 13

1.4 IEEE $802.15 .6 \ldots \ldots \ldots \ldots \ldots \ldots$

1.4 .1 Physical layer . . . . . . . . . . . . . . . . 15

1.4.2 Medium access control layer (MAC) . . . . . . . . . . . . 17

MAC frame format . . . . . . . . . . . . . . . 18

MAC channel Access Mechanism . . . . . . . . . . . . . . 18

IEEE 802.15.6 CSMA/CA mechanism . . . . . . . . . . . . . . 20

1.5 Research work . . . . . . . . . . . . . . . . . 22

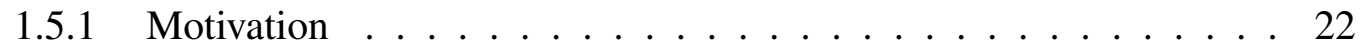

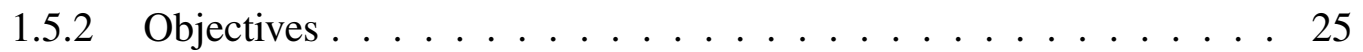

1.5.3 Main contributions . . . . . . . . . . . . . . 27

1.6 Thesis organization $\ldots \ldots \ldots \ldots \ldots \ldots \ldots \ldots \ldots \ldots \ldots$ 
2 Related Work 34

2.1 PRACH overload . . . . . . . . . . . . . . . . . . . . . . . . . . . . . 34

2.2 Earlier work on overlays in LTE . . . . . . . . . . . . . . . . . 38

2.2.1 Use of LTE overlay for IEEE 802.15.4 in VANET scenarios . . . . 38

2.2.2 Use of LTE overlay for priority based networks . . . . . . . . . . . 41

2.3 Chapter summary . . . . . . . . . . . . . . . 45

3 Non priority CSMA/CA overlay network to support vehicular traffic over LTE 46

3.1 Introduction . . . . . . . . . . . . . . . . 47

3.2 LTE-based VM2M overlay architecture . . . . . . . . . . . . . . . . . . . . 48

3.2.1 Service channel $(\mathrm{SCH}) \ldots \ldots \ldots$. . . . . . . . . . 49

3.2.2 VM2M overlay for control channel $(\mathrm{CCH}) \quad \ldots . . . . . . . .449$

3.2.3 VM2M random access procedure ............ 52

3.2.4 $\mathrm{H} 2 \mathrm{H}$ overload probability during random access handshake . . . . 53

3.2.5 VM2M overload calculation . . . . . . . . . . . . . 57

3.3 Performance of VM2M in the presence of $\mathrm{H} 2 \mathrm{H}$ traffic . . . . . . . . . . . . 59

3.3.1 Experiment 1: Combinations of preamble formats (PF) and configuration parameters $\left(c_{f}\right) \ldots \ldots \ldots 61$

3.3.2 Experiment 2: PRACH bandwidth partitioning for VM2M . . . . 83

3.3.3 Experiment 3: Priority based VM2M communications over LTE . . 93

3.4 Chapter summary . . . . . . . . . . . . . . . . . . . . . . 99

4 Priority based CSMA/CA overlay network over LTE 104

4.1 Introduction . . . . . . . . . . . . . . . . 105

4.2 PRACH architecture and random access procedure . . . . . . . . . . 109

4.2.1 Random access procedure . . . . . . . . . . . . . . 110

4.3 PM2M overlay network over PRACH . . . . . . . . . . . . . . . . 113

4.3.1 PM2M random access procedure . . . . . . . . . . . . 114

4.3.2 Physical and MAC layer . . . . . . . . . . . . . . . . . . 114

4.3 .3 CSMA/CA mechanism . . . . . . . . . . . . . . . . 117

4.3 .4 Priority mapping . . . . . . . . . . . . . . . 118

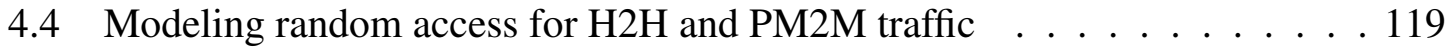

4.4.1 H2H PRACH overload during preamble collision . . . . . . . . . 121

4.4.2 PM2M overload calculation . . . . . . . . . . . . . . 122

4.4.3 Performance of $\mathrm{H} 2 \mathrm{H}$ traffic in the presence of overlay Network . . 124

4.5 Modeling PM2M overlay network backoff procedure with backoff error . . 126

4.6 Performance evaluation of PM2M overlay network . . . . . . . . . . . 141

4.6.1 Performance without backoff error . . . . . . . . . . . . . . . . 142

4.6.2 Performance comparison with reference approach for without back-

off error . . . . . . . . . . . . . . . . . . . 143

Performance evaluation with backoff error . . . . . . . . . . . 149 
4.6.3 Summary: Comparison between PM2M overlay network with and without Backoff error in the presence of $\mathrm{H} 2 \mathrm{H}$ traffic . . . . . . . . 151

4.7 Chapter summary . . . . . . . . . . . . . . . . 153

5 Conclusion $\quad 156$

$\begin{array}{lll}\text { Appendix A } & \text { Publications } & 160\end{array}$

A.1 Journal Papers . . . . . . . . . . . . . . . . . . . . . 160

A.2 Conference Papers . . . . . . . . . . . . . . . . 161

$\begin{array}{lll}\text { Appendix B Abbreviations and Symbols } & 163\end{array}$

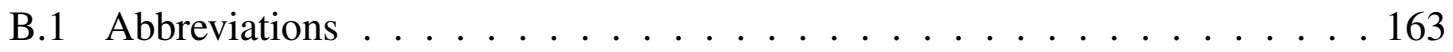

B.2 Symbols and Corresponding Descriptions . . . . . . . . . . . . 166

$\begin{array}{ll}\text { Bibliography } & 171\end{array}$ 


\section{List of Figures}

1.1 M2M communications for smart city scenarios through LTE $\ldots . . . . . .2$

1.2 Four way handshaking between MT terminal and eNodeB according to [53] ............................. 4

1.3 LTE frame structure with available resources . . . . . . . . . . . 8

1.4 PRACH resource configurations, after [74] . . . . . . . . . . . . . . 9

1.5 PRACH preamble format, after [74] . . . . . . . . . . . . 10

1.6 Vehicular communication through LTE. . . . . . . . . . . . . . . 12

1.7 Frequency bands used in IEEE 802.15.6. [44] . . . . . . . . . . . . . . 16

1.8 IEEE 802.15.6 superframe structure for beacon mode with beacon period boundaries. . . . . . . . . . . . . . . . . 19

1.9 IEEE 802.15.6 CSMA Slot Structure. [69] . . . . . . . . . . . . . 21

3.1 LTE and VM2M Overlay Superframe with PF 2 in TDD Mode (2 ms Sub-

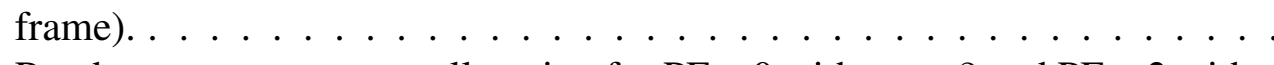

3.2 Random access resource allocation for $\mathrm{PF}=0$ with $c_{f}=2$ and $\mathrm{PF}=2$ with

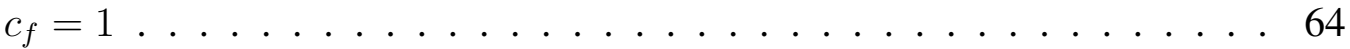

3.3 Performance of $\mathrm{H} 2 \mathrm{H}$ handshake for $P F=0 c_{f}=2 \ldots \ldots \ldots 64$

3.4 Performance of $\mathrm{H} 2 \mathrm{H}$ handshake for $P F=2$ with $c_{f}=1 \ldots \ldots 5$

3.5 Random access resource allocation for $P F=0$ with $c_{f}=3,5$ and $P F=2$

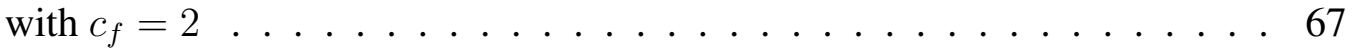

3.6 Performance of $\mathrm{H} 2 \mathrm{H}$ handshake for $P F=0$ with and $c_{f}=3 \ldots \ldots 8$

3.7 Performance of $\mathrm{H} 2 \mathrm{H}$ handshake for $P F=0$ with $c_{f}=5 \ldots \ldots$. . . . 69

3.8 Performance of $\mathrm{H} 2 \mathrm{H}$ handshake for $P F=2$ with and $c_{f}=2 \ldots \ldots 71$

3.9 Performance parameters for M2M overlay network for $P F=0$ and $c_{f}=2 \quad 76$

3.10 Performance parameters for M2M overlay network for $P F=2$ and $c_{f}=1 \quad 77$

3.11 Performance parameters for M2M overlay network for $P F=0$ and $c_{f}=3 \quad$. 79

3.12 Performance parameters for M2M overlay network for $P F=0$ and $c_{f}=5 \quad$. $\quad 80$

3.13 Performance parameters for M2M overlay network for $P F=2$ and $c_{f}=2 \quad 81$

3.14 Random access resource allocation for $\mathrm{PF}=2$ with $c_{f}=1 \ldots \ldots 84$

3.15 Performance of $\mathrm{H} 2 \mathrm{H}$ handshake for $P F=2, N_{M}=4 c_{f}=1 \ldots$. . . . 87 
3.16 Performance parameters for VM2M overlay network for $P F=2, N_{M}=4$ and $c_{f}=1 \ldots \ldots \ldots \ldots \ldots \ldots$

3.17 Performance of $\mathrm{H} 2 \mathrm{H}$ handshake for $P F=2, N_{M}=8, c_{f}=1 \ldots$. . . . 89

3.18 Performance parameters for VM2M overlay network for $P F=2, N_{M}=8$ $c_{f}=1 \ldots \ldots \ldots \ldots \ldots$

3.19 Performance of $\mathrm{H} 2 \mathrm{H}$ handshake for $P F=2, N_{M}=12, c_{f}=1 \ldots$. . . 91

3.20 Performance parameters for VM2M overlay network for $P F=2, N_{M}=12$,

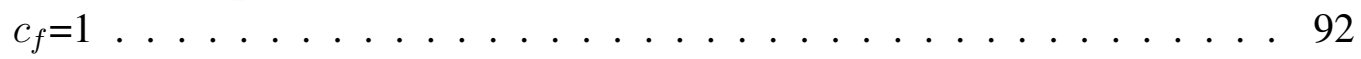

3.21 Performance of $\mathrm{H} 2 \mathrm{H}$ handshake for $P F=2$ with $c_{f}=1$ without priority. . 97

3.22 Performance parameters for M2M overlay network for $P F=2$ and $c_{f}=1$ without priority . . . . . . . . . . . . . . . . 101

3.23 Performance of $\mathrm{H} 2 \mathrm{H}$ Handshake for $P F=2, N_{M}=8 c_{f}=1$ with VM2M

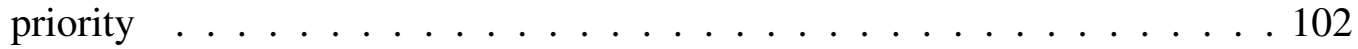

3.24 Performance of VM2M Overlay Network for $P F=2, N_{M}=8$ and $c_{f}=1$ with VM2M priority . . . . . . . . . . . . . . . 103

4.1 PRACH resource configurations, after [74] . . . . . . . . . . . 111

4.2 Four way handshaking between MT terminal and eNodeB according to [53] . . . . . . . . . . . . . . . . . . . . . 112

4.3 LTE and PM2M Overlay Superframe with PF 2 in TDD Mode (2 ms Subframe . . . . . . . . . . . . . . . . . . . 113

4.4 Random access resource allocation for $\mathrm{PF}=2$ with $c_{f}=5 \ldots \ldots . \ldots 125$

4.5 Performance of $\mathrm{H} 2 \mathrm{H}$ traffic for $P F=2 c_{f}=5 \ldots \ldots . . . \ldots 126$

4.6 Markov chain for $U P_{k}$, modified from [72] . . . . . . . . . . . . 131

4.7 Extended Markov chain for $U P_{k}$ modified from [72] . . . . . . . . . . . 136

4.8 Probability of successful access to the medium by each node without backoff error. . . . . . . . . . . . . . . . . . . . . . . . . 144

4.9 Mean Backoff time of each node without backoff error. . . . . . . . . . . . 144

4.10 Medium access probability by each node without backoff error. . . . . . 145

4.11 Normalized throughput by each node without backoff error. . . . . . . . . 145

4.12 Mean number of backoff attempt by each node without backoff error. . . . 146

4.13 Performance evaluation of PM2M overlay and reference approach for without consideration of backoff error . . . . . . . . . . . . . . . . . 148

4.14 Performance evaluation of each node of PM2M overlay for lower traffic class $T C_{0}$ with backoff error . . . . . . . . . . . . . . . . 151

4.15 Performance evaluation of each node of PM2M overlay for higher traffic class $T C_{1}$ with backoff error . . . . . . . . . . . . . . 152 


\section{List of Tables}

1.1 Difference between VANET implementation on LTE and IEEE 802.11p . . 11

1.2 Frequency band and bandwidth of IEEE 802.15.6 [63] . . . . . . . . . 17

1.3 The illustration of main contributions in this thesis. . . . . . . . . . . 28

1.4 The illustration of main contributions in this thesis. . . . . . . . . . . . 29

3.1 Implementation Parameters of VM2M overlay Network . . . . . . . . . . . 61

4.1 IEEE 802.15.6 access categories (AC) with user priorities(UP) and contention window $(\mathrm{CW})$ values . . . . . . . . . . . . . . . 118

4.2 Implementation Parameters of PM2M overlay Network . . . . . . . . . . . 142

4.3 Comparison between reference approach and PM2M overlay . . . . . . . . 147 


\section{Chapter 1}

\section{Introduction}

Machine to Machine (M2M) or Machine type communication (MTC) is a recent data communication paradigm that supports data communication between smart electronic devices without direct human supervision. In M2M communication a large number of intelligent devices are connected by wired or wireless links, interact with each other and exchange information. The applications of M2M are widely seen in many areas such as smart grids, intelligent transportation systems, e-healthcare, home area networks, environmental monitoring, smart cities and industrial automation [80]. MTC or M2M communications was designed to fulfil the requirements of transmitting the huge volume of low data rate messages. It can adopt a cellular network to extent the coverage. The cellular networks large coverage feature makes it a promising option for M2M communications. MTC networks are projected to have a huge number of mostly stationary terminals that are always active. Cellular networks are optimized for high performance human to human communications $(\mathrm{H} 2 \mathrm{H})$ and not well suited for M2M communications because of their high volume and different traffic characteristics. In recent years, a number of possible solutions were pro- 
posed for cellular MTC where each MTC terminal connects directly to a $3 \mathrm{G}$ or $4 \mathrm{G}$ cellular network. Fig. 1.1 shows a smart city scenario where applications are connected directly to cellular network, through which it communicates with the designated MTC server.

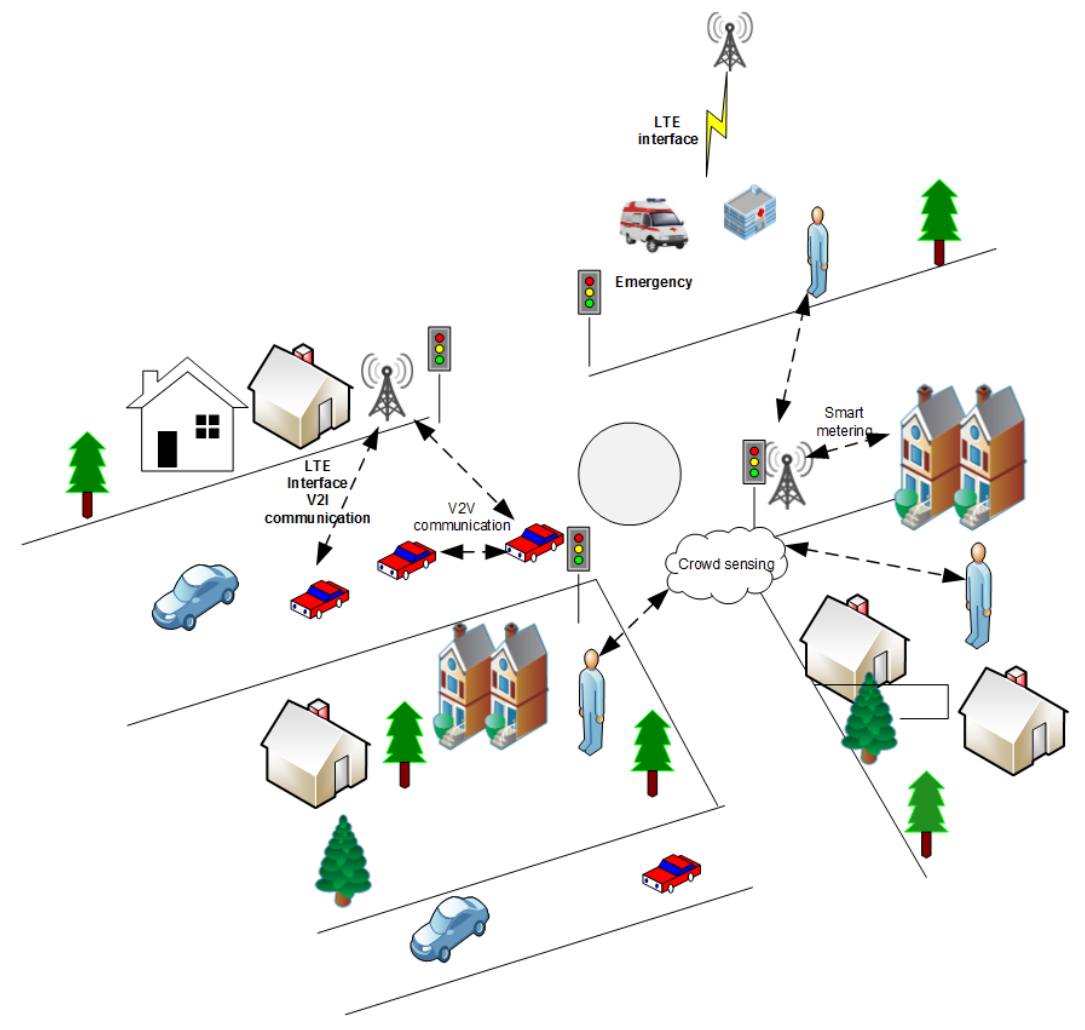

Figure 1.1: M2M communications for smart city scenarios through LTE

Long-Term Evolution (LTE) commonly referred as 4G LTE, is a standard for cellular wireless communication which can offer solutions for M2M communications over higher distances and speeds. Its high data rate, low latency and large coverage features give LTE advantages over other technologies for M2M communications. In M2M communications the devices could communicate with each other with numbers of wireless communication technology such as Zigbee (IEEE 802.15.4), Bluetooth, WiFi, IEEE 802.15.6 and cellular networks. The purpose of this dissertation work is to investigate the perspective of the 
application areas of machine to machine (M2M) communications specifically to build smart city. We wanted to have some networks over the city that will have ubiquitous coverage, which is why we consider cellular infrastructure. In this thesis work we propose a M2M overlay network over LTE PRACH (physical random access channel) so that M2M data will be transmitted in parallel with $\mathrm{H} 2 \mathrm{H}$ traffic without overloading the access network. The overlay network could be used for many application areas of a smart city. We have proposed non-priority CSMA/CA overlay (VM2M) and priority based CSMA/CA overlay (PM2M) over LTE. Based on these architectures first, we conduct a case study in a vehicular context and an M2M architecture is introduced for the intelligent vehicular machine to machine (VM2M) networks. Secondly, to introduce priority over different M2M traffic classes we proposed a priority based machine to machine (PM2M) overlay network which could be used for wide deployment scenarios.

The rest of the chapter is organized as follows: Features of LTE is discussed in section 1.1. The details about VANET (Vehicular Ad hoc network) and IEEE 802.15.6 with challenges are discussed in section 1.2 and 1.4 respectively. The thesis motivation, objectives and main contributions are presented in Section 1.5. Finally, the organization of this thesis is presented in Section 1.6.

\subsection{LTE}

The features of LTE is described in this section. 


\subsubsection{Random access procedure}

Communications in LTE are established over physical random access channel (PRACH). Each node wishing to communicate with base station needs to complete four-way handshake which starts by transmission of preambles. In LTE, mobile terminals (MT) which do not have allocated uplink radio resources perform random access (RA) procedure to connect to the network before they transmit data. The RA procedure is performed through the RACH [53] and consists of four-way handshaking with the eNodeB as shown in Figure 1.2.

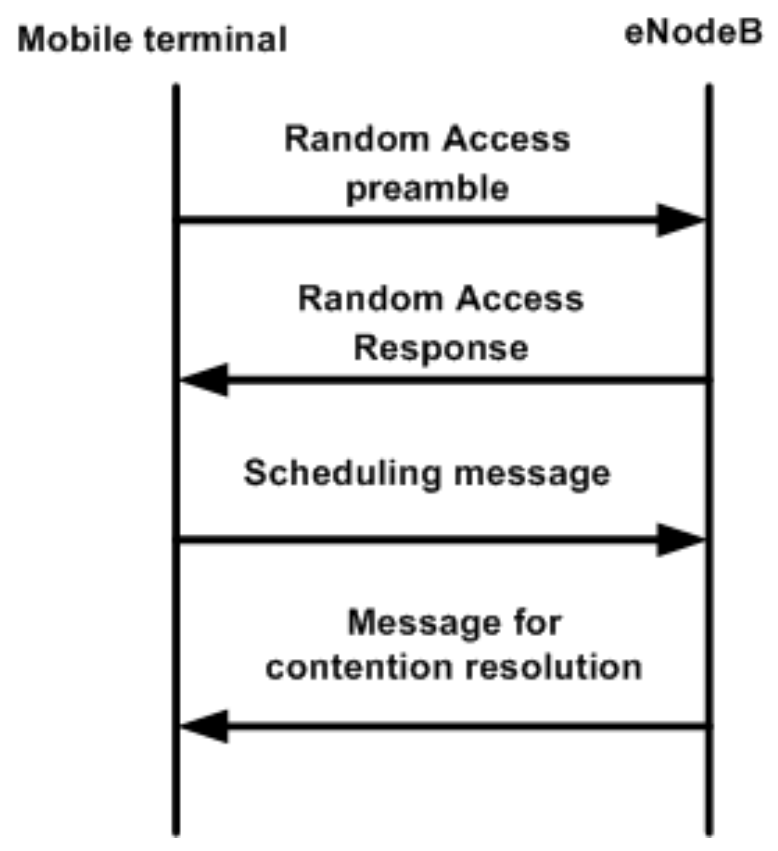

Figure 1.2: Four way handshaking between MT terminal and eNodeB according to [53]

The RA can be contention based or contention free (with reserved 10 preambles for time-critical situation like handover). The contention-based 4-way handshaking procedure of RA contains following steps: 
Step 1: The MT randomly selects the preamble from available 54 preamble sequences (remaining 10 preambles are reserved for handover calls) and transmits it over PRACH to eNodeB.

Step 2: The eNodeB transmits an RA response (RAR) to MT through the downlink shared channel (PDSCH). RAR contains temporary Cell Radio Network Temporary Identifier (CRNTI) identity information. It also contains scheduling information for the third step.

Step 3: Then, MT sends its CRNTI and scheduling information to eNodeB through a physical uplink shared channel (PUSCH) radio resources assigned in step 2.

Step 4: Finally, the eNodeB responds with the confirmation of the identity of MT and finishes the contention procedure.

Unfortunately, first and third steps of handshaking are prone to collisions and overload conditions due to the limited number of preambles. This prevents completion of RA handshake [61] for a large number of short data transmissions from vehicles where SINR (Signal to interference noise ratio) at eNodeB is violated. In a nutshell use of four-way handshaking procedure of LTE for massive network access in CCH is inefficient and leads to network overload. This has motivated our research efforts towards VANET overlay architecture where $\mathrm{CCH}$ is implemented as an overlay in PRACH.

\subsubsection{RACH overload control approaches}

The concurrent access of massive amount of M2M devices to the access network of LTE causes overload and congest the network. Due to congestion, the probability of collision 
becomes high and it causes the failure rate and access delay high. Many researchers and 3GPP [77] aims to reduce the overload condition and the efficient utilization of available resources. 3GPP classifieds the random access overload control scheme into two categories: push-based and pull-based.

In push-based approaches the devices can transmit the data to the network whenever they have data to transmit. The challenge of this approach is to control the channel access opportunity by a massive number of M2M devices. Many of the researches propose many solutions to control the overload situation of push based-approach for example, Access class barring (ACB) [22, 54], dynamic resource allocation between $\mathrm{H} 2 \mathrm{H}$ and M2M devices [47], backoff scheme [23], separate RACH resources between $\mathrm{H} 2 \mathrm{H}$ and M2M. All those schemes use 4-way handshaking mechanism to access the PRACH and most of them use Slotted ALOHA as the medium access mechanism.

In pull-based approach the eNodeB restricts the number of M2M devices trying to access the network. The devices can only transmit data when they are invited to by the network. In this way, it controls the massive numbers of devices trying to access the network and control the overload and congestion. Example solutions of pull-based approach is paging and group paging $[55,84]$. In paging, eNodeB can only page 16 devices in a single paging message and thus has to spend a long time to page every device. To reduce the signalling overhead group paging scheme was proposed where on behalf of every group of M2M devices a group leader is selected and only a single group page is send to trigger that group. This approach also follows 4-way handshaking mechanism to access the network. The disadvantages of pull-based approach are it decrease the number of M2M devices which are allowed to access the network. The group leader still needs to follow 4 
step handshaking mechanism which creates signalling overhead. Selection of group leader among M2M devices is the challenging issue for grouping methods. If the size of the group is massive it can cause the probability of collision high, results low access success probability and high access delay. It also has the disadvantages of low resource utilization issue compared with push-based approach.

\subsubsection{Frame structure, configuration and PRACH resources in LTE}

Available bandwidth resources for LTE cell are divided in time and frequency domain matrix as shown in Fig. 1.3. Time access is organized in frames that last $10 \mathrm{~ms}$ and consists of 10 subframes with duration of $1 \mathrm{~ms}$ each. Subframes can be divided into two $0.5 \mathrm{~ms}$ slots. In the frequency domain resources are grouped in units of 12 OFDM subcarrier with a total bandwidth of $180 \mathrm{kHz}$. Basic access unit either for random or scheduled access is resource block (RB) which consists of 12 subcarriers over one subframe duration of $1 \mathrm{~ms}$. For control channels, finer granularity is available during one slot. Smallest resource unit for control channels is resource element (RE) and consists of one subcarrier for the duration of one OFDM symbol.

Cell bandwidth can be configured in FDD or TDD. According to time division duplex (TDD) unpaired spectrum frame structure type 2 , subframes 0 and 5 is always reserved for downlink transmission and other subframes can be used for uplink or downlink transmission. Minimum PRACH bandwidth is composed of six resource blocks in frequency with a bandwidth of $1.080 \mathrm{MHZ}$ and one subframe over two frame time. RACH resources are shared by all user equipment, which results congestion in the network and shortage of resources. Adaptation to larger random access traffic is possible through 16 PRACH con- 


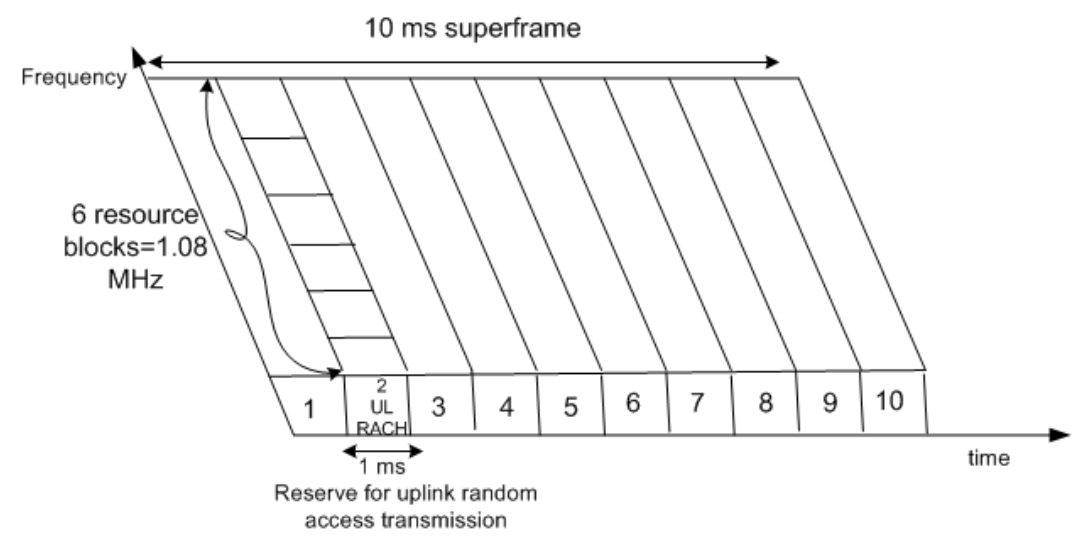

Figure 1.3: LTE frame structure with available resources

figurations as shown in figure 1.4. For low traffic intensity and small system bandwidth one PRACH resource over 2 frames may be sufficient (TDMA configurations $0,1,2$ and 15). As traffic increases PRACH resource occurs once per frame (TDMA configurations 3, 4 and 5). Further, as traffic increases PRACH resource can occur once per 5 subframes (TDMA configurations 6, 7 and 8) or once per 3 subframes (TDMA configurations 9, 10 and 11). Although previous PRACH allocations avoided interference at a granularity of 3 cells for high traffic dense PRACH allocations bring the possibility of interference since PRACH resource occurs on every second subframe (configurations 12 and 13) or on every subframe in a frame (configuration 14). However, care must be take to avoid a possibility of interference between PRACH and PUSCH channels among cells at close distances.

\subsubsection{Preambles formats}

To accommodate different attenuations for various cell sizes and propagation delays LTE defines 4 different preamble formats (PF), denoted as 0,1,2 and 3. High attenuation is addressed by increased preamble duration. The cyclic prefix (CP) and guard time (GT) 


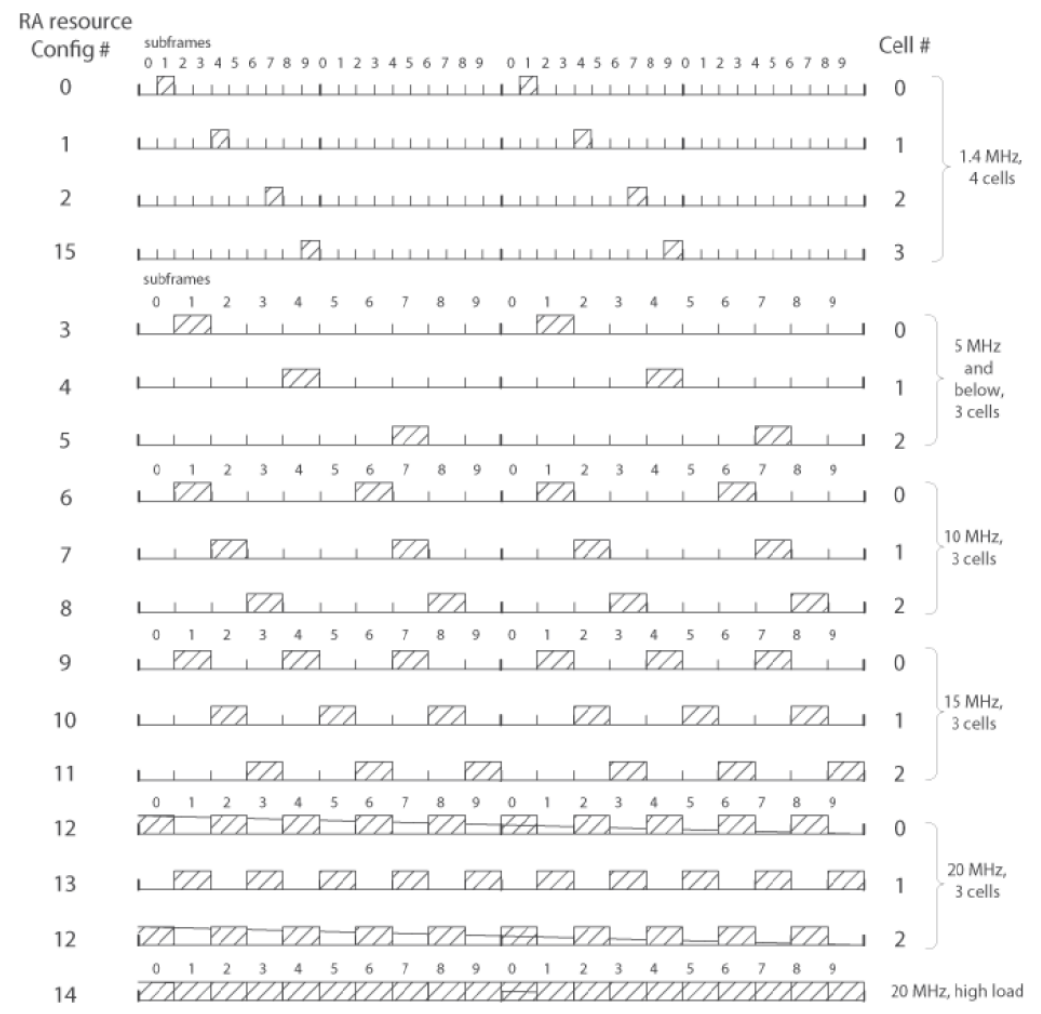

Figure 1.4: PRACH resource configurations, after [74]

are used at the beginning and at the end of the PRACH resource to avoid delays and interference with the adjacent subframes as shown in figure 1.5 . Format 0 preamble duration is $800 \mu$ s and it is accompanied with $200 \mu$ s of joint CP and GT. For formats 2 and 3 preamble duration is $1600 \mu \mathrm{s}$. Formats 1 and 2 fit in 2 consecutive subframes and format 3 fits in 3 consecutive subframes. Preambles used in first random access are mutually orthogonal Zadoff-Chu(ZC) sequences which are derived from a single base sequence by adding cyclic shifts. One base sequence gives 64 sequences. A Larger number of preamble sequences can be obtained by using two or more base sequences. Among 64 preambles $N_{h}=10$ sequences are allocated for non contention manner for handover calls and remaining $N_{i}=54$ sequences are allocated for contention mode. 


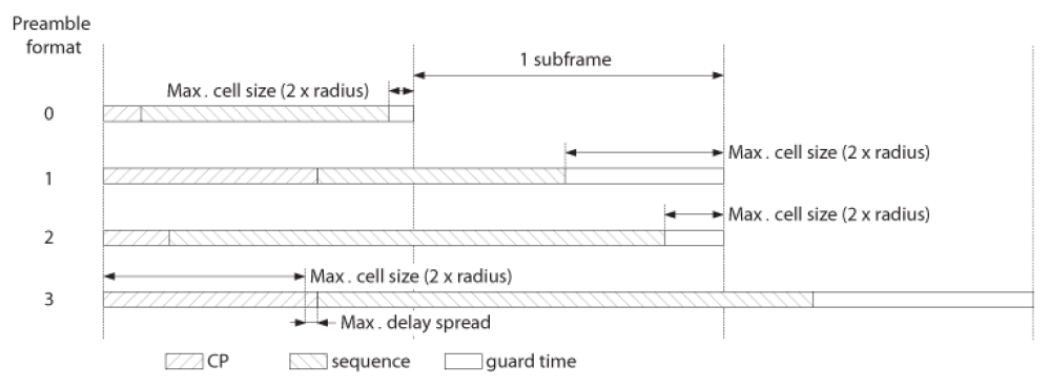

Figure 1.5: PRACH preamble format, after [74]

\subsection{Vehicular Adhoc network (VANET)}

VANET is a kind of mobile ad hoc network that supports communication among vehicles. The vehicular network provides a variety of applications like road safety, infotainment and telematics. These applications reduce road accidents and improve traffic management. Vehicular Ad hoc network (VANET) supports communication between vehicle-to-vehicle $(\mathrm{V} 2 \mathrm{~V})$ and vehicle-to-roadside unit (V2R) to transfer safety messages through the dedicated control channel $(\mathrm{CCH})$ and other messages like infotainment or telematics through one or more service channels (SCH). IEEE 802.11p standard for VANETs enables V2V and V2R communications through the onboard unit (OBUs) which is vehicles interface to VANET can also be considered as a vehicular machine to machine (VM2M) device to take part in vehicular communications.

\subsubsection{Limitations of IEEE 802.11p and features of LTE}

A VANET usually follows the DSRC (Dedicated Short Range Communications) standard which deploys the IEEE 802.11p standard for wireless communications [76]. IEEE 802.11p (also referred to as Wireless Access for the Vehicular Environment (WAVE), works 
well in urban areas with a dense vehicular population with low speeds and small V2V and V2R distances. However, in suburban, rural areas or highways where the speed limit is higher and the distance between the vehicles and RSUs are larger, IEEE 802.11p is not an optimal solution. The standard is not well suited for VM2M communications due to unbounded delay, limited radio range, low scalability, and lack of persistent infrastructure [7]. It has been shown to be prone to congestion, early saturation and rapid decrease of transmission rate under high vehicle density, large RSU coverage area and large distances between RSUs and OBUs [58]. In suburban, rural or highways where distances between OBUs and RSUs are large LTE could be a preferable solution. LTE could provide high data rate and large coverage and it has a very sophisticated physical layer with high data rates. Difference between VANET implementation on proposed VM2M overlay network on LTE and IEEE $802.11 \mathrm{p}$ networks for $\mathrm{CCH}$ and $\mathrm{SCH}$ are shown in table 1.1 .

Table 1.1: Difference between VANET implementation on LTE and IEEE 802.11p

\begin{tabular}{|l|l|l|}
\hline LTE & SCH & CCH \\
\hline IEEE 802.11p & $\begin{array}{l}\text { Scheduled access after winning } \\
\text { Contention on RACH. }\end{array}$ & $\begin{array}{l}\text { Overlay of PRACH, preamble } \\
\text { separation, reduce 4 way to 2 } \\
\text { way handshake. }\end{array}$ \\
\hline
\end{tabular}

All of the above features have motivated us to consider the possibility of using LTE random access channel as a candidate for $\mathrm{CCH}$ in VANETs for suburban, rural areas and highways. To overcome the limitations of IEEE 802.11p for M2M communications we consider CSMA/CA medium access control mechanism similar to the one used in IEEE 802.15.4. Conceptual architecture of a VANET implemented via LTE is shown in Figure 1.6. 


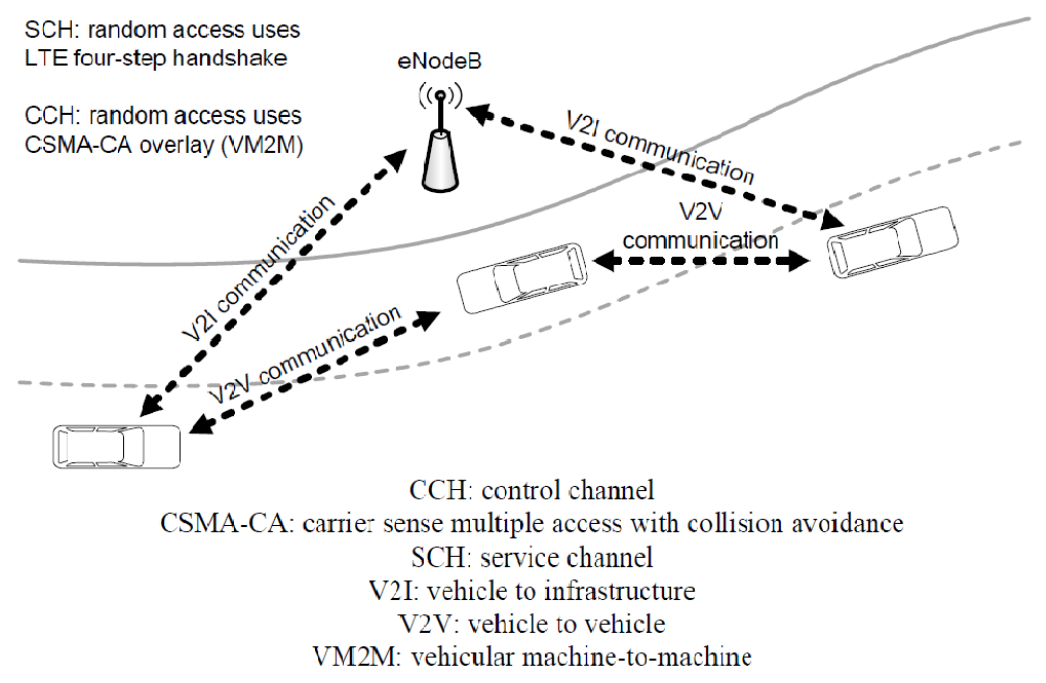

Figure 1.6: Vehicular communication through LTE.

\subsection{IEEE 802.15.4}

The IEEE 802.15.4 is a standard that provides low complexity, low power, low data rate, and energy efficient battery powered devices. Wireless Personal Area Networks (WPAN) and Wireless Sensor Networks (WSN) are low data rate and short-range wireless networks that build on the IEEE 802.15.4 standard. All of the features of IEEE 802.15.4 have motivated us to implement proposed overlay networks MAC layer with CSMA/CA similar to the one used in IEEE 802.15.4.

These networks are infrastructure less and used for applications based on their requirements for data rate, power consumption and QoS. The IEEE 802.15.4 works on three frequency bands: $868 \mathrm{MHz}, 915 \mathrm{MHz}$ and $2.45 \mathrm{GHz}$ ISM band and used by various WPAN standards such as IEEE 802.15.1, 802.15.3 and also by microwave ovens. The maximum data transmission rate in this band is $250 \mathrm{kbps}$. In WPAN networks, there is a central controller called PAN coordinator that builds personal area networks with other nodes within 
a small space. PAN coordinator mainly supports two types of topologies: star and peerto-peer. In star topology, a device must go through PAN coordinator to communicate with other devices. In peer-to-peer topology, a device can communicate directly with others if they are in the communication range.

\subsubsection{MAC channel access mechanism}

The way of communication between device to device or device to PAN coordinator depends on whether the network supports beacon or not. The beacon synchronizes the whole networks transmission procedure The IEEE 802.15.4 standard defines two MAC channel access mechanisms:

- Beacon-enabled channel access: In this mode, the coordinator periodically transmits a beacon frame. The PAN coordinator divides the channel time. The time between two beacon frames is called beacon interval (BI) or superframe. Each superframe starts with a beacon frame and is divided into an active and inactive portion. All the devices communicate with their PAN coordinator during the active portion of the superframe. They send their data to the coordinator through uplink transmission and receive data from the coordinator through downlink transmission.

- Non-beacon-enabled channel access: Non-beacon-enabled networks use non-slotted CSMA-CA mechanism

\section{CSMA/CA medium access control mechanism}

Uplink transmissions in the star topology use the CSMA-CA mechanism in beaconenabled mode. The CSMA/CA mechanism, starts when the queue holds at least a packet to 
transmit. In this mechanism the transmission only occurs at the boundary of backoff slots. At first, a random number is generated at the backoff periods in the range of $0 \ldots 2^{B E}-1$, where $\mathrm{BE}=$ backoff exponent. The value is then decremented at the boundary of each backoff period, which is called Random Backoff Countdown or RBC. During RBC, the channel is not sensed and if it is not available backoff countdown is not frozen. If the current superframe does not have enough time to finish the $\mathrm{RBC}$, the station needs to wait until the next superframe active period. The counter is kept frozen only in the inactive portion of the superframe and resumes again in the active portion of the next superframe. If the value of $\mathrm{RBC}$ reaches zero before transmitting the packet the algorithm needs to check the status (idle or not) of the channel. This is done by listening to the channel by Clear Channel Assessment (CCA) procedure in two backoff periods. Before starting the CCA the algorithm also needs to check if the current CAP has enough time to complete the necessary number of CCA checks, the packet transmission time, and acknowledgement. If there is enough time for all these activities the algorithm starts CCA check. Otherwise, the station needs to wait until the active period of the next superframe.

\subsection{IEEE 802.15.6}

To implement proposed overlay in wide deployment scenarios of smart city and to define priority among different M2M applications we adopt IEEE 802.15.6. IEEE 802.15.6 is the short-range priority based standard which provides low power and highly reliable wireless communication systems. IEEE 802.15.6 is the standard for wireless body area networks (WBAN) but it is not only dedicated to medical and health applications. It is also designed to support consumer electronics, sports, military applications and advanced 
medical equipment which enables the consumer to have power efficient, reliable and interference free solutions to deploy a variety range of devices [20]. IEEE 802.15.6 supports different data rates for different applications and up to $10 \mathrm{Mbps}$ data rate is required. It's design mechanism supports it to consider effects of portable antennas. It is capable to minimize the specific absorption rate (SAR) of the body through radiation pattern shaping. It also supports changes in characteristics due to the movement of the users. In the following sub sections we will describe the main features of IEEE802.15.6 standard including PHY and MAC layers specifications.

\subsubsection{Physical layer}

In IEEE 802.15.6 nodes are partitioned into the physical layer (PHY) and medium access control layer (MAC). The purpose of IEEE 802.15.6 was to define new PHY and MAC layer for applications such as healthcare. Its target was to provide quality of service for communication of low power devices in, on and around the human body. The standard supports different frequency bands (PHYs) and the selection of the PHY depends on the application requirements. The standard also defines a sophisticated MAC layer with priority mechanism to control access to the medium. Medical Implant Communications Service (MICS) band is a licensed band with a frequency range from 402 to $405 \mathrm{MHz}$ and used for implant communication. WMTS (Wireless Medical Telemetry Services) is a licensed band used for medical telemetry system [9]. It also supports unlicensed, Industrial, Scientific and Medical (ISM) band that is used for high data rate applications whereas, the former MICS and WMTS supports low data rate applications. Although ISM supports high data rate it suffers from high interference by others wireless devices like IEEE 802.11 and 
IEEE 802.15.4 as they also operate in this license free frequency band. Fig. 1.7 shows the frequency bands used in IEEE 802.15.6 [44].

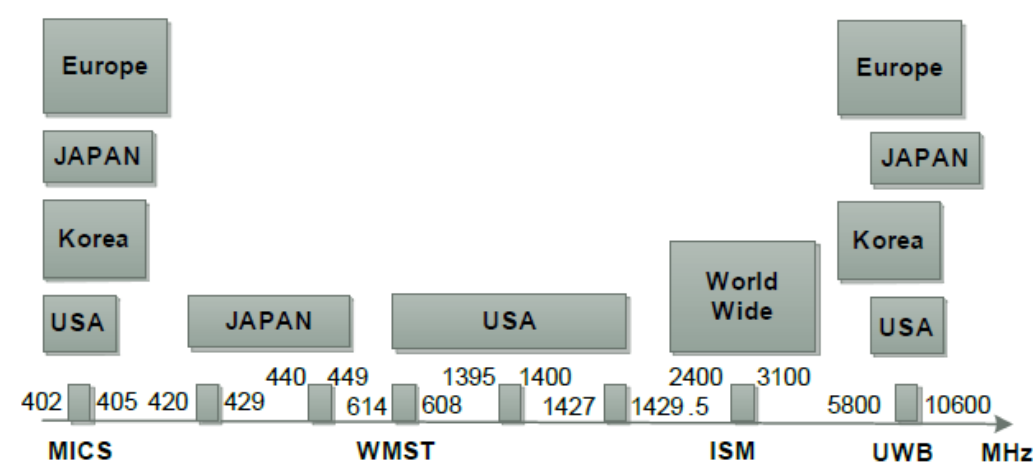

Figure 1.7: Frequency bands used in IEEE 802.15.6. [44]

IEEE 802.16.5 defines three physical layers i.e., Narrow band (NB), Ultra-wideband (UWB), and Human Body Communications (HBC) layers. The use of each layer depends on the application requirements. The main function of the physical layer is to transform physical layer service data units (PSDU) into physical layer protocol data units (PPDU). Table 1.2 shows the frequency bands and bandwidths of the different physical layers according to IEEE 802.15.6 [63].

Narrow band layer is the responsible for activation and deactivation of radio transceiver, data transmission, clear channel assessment and transformation of PSDU into PPDU. In healthcare system, NB layer is used for on-body communication due to the high power of the nodes in NB communications. Ultra-wideband PHY operates in low and high frequency bands and each band is divided in to channels. The low band consist of 3 channels and among them only channel 2 with $3993.6 \mathrm{MHz}$ frequency band is considered as a mandatory channel. The high band consists of 8 channels. Channel number 7 with a central frequency of $7987.2 \mathrm{MHz}$ is considered as a mandatory channel and a typical UWB device should 
Table 1.2: Frequency band and bandwidth of IEEE 802.15.6 [63]

\begin{tabular}{|c|c|}
\hline \multicolumn{2}{|c|}{ Human-Body Communication } \\
\hline Frequency & Bandwidth \\
\hline $16 \mathrm{MHz}$ & $4 \mathrm{MHz}$ \\
\hline $27 \mathrm{MHz}$ & $4 \mathrm{MHz}$ \\
\hline \multicolumn{2}{|c|}{ Narrowband Communication } \\
\hline Frequency & Bandwidth \\
\hline $402-405 \mathrm{MHz}$ & $300 \mathrm{kHz}$ \\
\hline $420-450 \mathrm{MHz}$ & $300 \mathrm{kHz}$ \\
\hline $863-870 \mathrm{MHz}$ & $400 \mathrm{kHz}$ \\
\hline $902-928 \mathrm{MHz}$ & $500 \mathrm{kHz}$ \\
\hline $956-956 \mathrm{MHz}$ & $400 \mathrm{kHz}$ \\
\hline $2360-2400 \mathrm{MHz}$ & $1 \mathrm{MHz}$ \\
\hline $2400-2438.5 \mathrm{MHz}$ & $1 \mathrm{MHz}$ \\
\hline \multicolumn{2}{|c|}{ UWB Communication } \\
\hline Frequency & Bandwidth \\
\hline $3.2-4.7 \mathrm{GHz}$ & $499 \mathrm{MHz}$ \\
\hline $6.2-10.3 \mathrm{GHz}$ & $499 \mathrm{MHz}$ \\
\hline
\end{tabular}

support at least one mandatory channel. UWB's low implementation complexity and low power consumption support it fulfil the requirements of MICS band. HBC layer support the devices that operates in 16 and $27 \mathrm{MHz}$ central frequency bands. HBC uses electric field coupling and responsible for packet structure, modulation and preamble.

\subsubsection{Medium access control layer (MAC)}

IEEE 802.15.6 defines a sophisticated MAC layer which is responsible to control channel access. The standard supports time division multiple access (TDMA), carrier sense multiple access with collision avoidance (CSMA/CA), and the combination of TDMA and CSMA/CA to meet the application requirements to access the medium [70, 71]. The MAC 
protocols frame format, medium access mechanism with techniques are described below.

\section{MAC frame format}

The MAC sub layer is responsible for transmitting MAC frame known as MAC Protocol Data Units (MPDU) to physical layer service access point (SAP). After receiving the data frame, the physical layer passes it to the MAC sub layer and then it passes the MSDU to the MAC client. Each of the MAC frames is composed of fixed length MAC header, variable size MAC frame body and fixed length frame check sequence (FCS) [73].

\section{MAC channel Access Mechanism}

In IEEE 802.15.6, the MAC layer channel is divided into a fixed length of beacon frame or super frames and each superframe is consist of an equal length of slots. To synchronize the device and associate the network a hub or network coordinator defines the size of the superframe time. A coordinator can operate in three different modes described below:

- Beacon mode with beacon period (superframe) boundaries: In beacon enabled mode, at the beginning of every superframe except inactive superframes a beacon is transmitted on the medium to provide the time reference allocation. Each of the superframe is divided into Access Phases (AP): Exclusive access phase (EAP) 1 and 2, random access phase (RAP) 1 and 2, Type I/II phase and contention access phase (CAP) as shown in figure 1.8. IEEE 802.15 .6 provides priority among the data traffic through AP where, EAP 1 and 2 is used for only high traffic class and RAP 1, 2 and CAP used for the regular traffic class. Type I/II phase are used for uplink, down link, blink and delay blink allocation interval. The standard allows to disable any 
of these AP depending on the requirements except RAP should be nonzero. EAP, RAP and CAP allows CSMA/CA or slotted Aloha medium access mechanism for the nodes through which they contend for the available resources. On the other hand, in type I/II phase resources are allocated for nodes through the polling mechanism. In our proposed PM2M network we consider beacon mode with beacon period. We only consider RAP phase and all other access phases are considered as zero time slots and CSMA/CA is used as contention based medium access mechanism. The details of CSMA/CA mechanism will be described below.

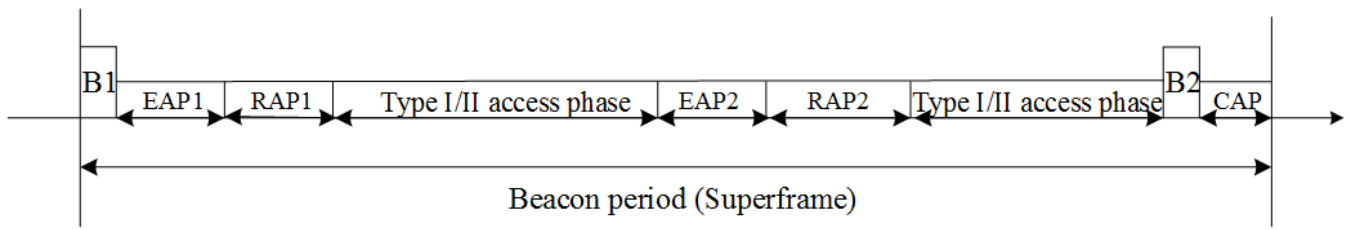

Figure 1.8: IEEE 802.15.6 superframe structure for beacon mode with beacon period boundaries.

- Non-beacon mode with superframe boundaries: In this mode, no beacon is transmitted and all the superframe is allocated for type 1 or 2 phase but not the both.

- Non-beacon mode without superframe boundaries: In this mode. no beacon is transmitted and no need to setup superframe and allocation of slots. In this mode the coordinator only allocates unscheduled Type I/II frames only.

MAC protocol can be categorized into three categories according to channel access mechanism: Random Access (RA) use CSMA/CA or slotted Aloha as the contention based medium access mechanism to allocate the resources for the nodes. Connectionless contention free mechanism uses an unscheduled polling mechanism to allocate the resources. 
Scheduled or connection oriented contention free access mechanism schedules the allocation of slots for upcoming superframes which is also known as 1-periodic or m-periodic allocation.

\section{IEEE 802.15.6 CSMA/CA mechanism}

The superframe starts immediately after receiving of the beacon frame. In CSMA/CA, before transmitting a frame a device senses the medium and transmits a frame only if the medium is idle and backoff counter becomes zero. If the medium is busy, the device has to wait for a predefined amount of time until the medium becomes idle. This protocol could avoid collisions as frame is transmitted only when the channel is idle. The CSMA protocol uses clear channel assessment (CCA) as the carrier sensing mechanism. At the beginning of access, a node calculates the backoff counter (BC) and contention window (CW) to determine when it obtains the chance to access the medium. The contention window values are set according to the following conditions:

- If the node is not previously allocated the contention values, $\mathrm{CW}$ is set as minimum $C W_{\min }$.

- After a successful transmission with acknowledgement the $\mathrm{CW}$ value is set back to $C W_{\min }$.

- If a successful transmission requires no acknowledgement the $\mathrm{CW}$ value remain unchanged.

- After an unsuccessful transmission or failed in transmission due to lack of receipt of acknowledging the $\mathrm{CW}$ values set as follows 
- In case of $m_{t h}$ number of unsuccessful transmission the $\mathrm{CW}$ value is remains same, where $\mathrm{m}$ is a odd number.

- Otherwise CW value is doubled for $n_{t h}$ number of unsuccessful transmission, where $\mathrm{n}$ is a even number. The $\mathrm{CW}$ value is doubled until it reaches the $C W_{\max }$.

The user priorities are differentiated by the values of the minimum and maximum contention window values. The $\mathrm{BC}$ is calculated as an integer random variable uniformly distributed over the interval $[1, \mathrm{CW}]$ where $C W \in\left(C W_{\min }, C W_{\max }\right)$. If the node sense medium is idle for SIFS amount of time it starts decrementing the BC. Any time it senses medium is busy it locks the $\mathrm{BC}$ and wait until the current transmission ends or medium becomes idle again. If the current superframe does not have enough time to complete the transmission it locks the $\mathrm{BC}$ and unlocks it when the medium is idle and has enough time to complete the transmissions. After completing the transmission, the $\mathrm{CW}$ value is set to minimum. The $\mathrm{BC}$ is decremented by one for each idle CSMA slot of duration equal to $\mathrm{pC}$ SMASlotLength as shown in figure 1.9. After backoff counter reaches 0 the node gets the chance to transmit the packets. Otherwise, the device has to wait for the next superframe.

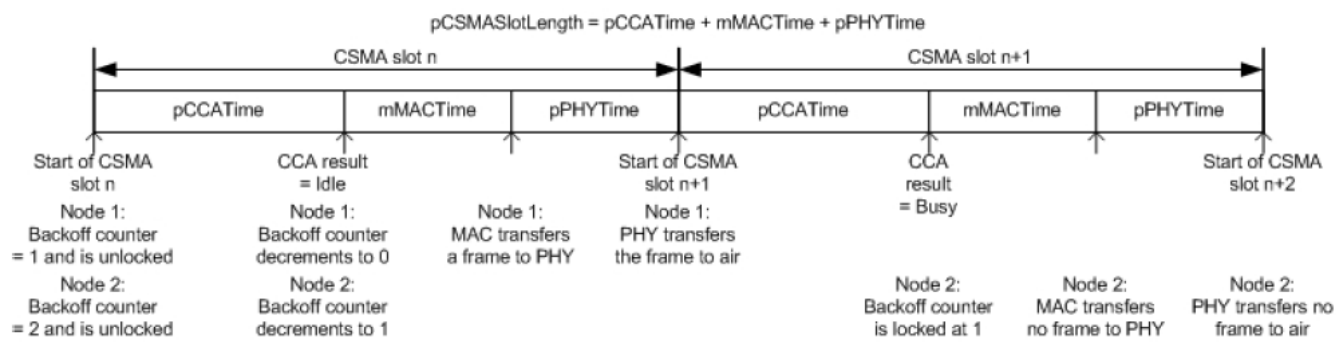

Figure 1.9: IEEE 802.15.6 CSMA Slot Structure. [69] 


\subsection{Research work}

Machine to machine (M2M) or machine type communications (MTC) support data communications among smart electronics devices without direct human supervision. M2M communications were designed to fulfil the requirements of transmitting the huge volume of low data rate messages by adopting communication channel. The cellular networks like LTE's large coverage feature make it a promising option for M2M communications. In this thesis work, we first investigate the potential M2M communication challenges in the application areas of smart city specifically for smart transportation system while adopting LTE as a communication channel. We then propose non-priority and priority based CSMA/CA overlay networks over LTE for wide deployment applications of smart city.

\subsubsection{Motivation}

Cellular networks are optimized for a large number of high performance mobile devices (e.g. phones) conveying human to human $(\mathrm{H} 2 \mathrm{H})$ communications with mostly downlink. $\mathrm{M} 2 \mathrm{M}$ networks are designed to have a large number of stationary or mobile terminals with uplink data flow. Direct implementation of M2M systems on the random access channel $(\mathrm{RACH})$ in LTE would result in an inefficient and non-scalable system that is difficult to extend to future M2M applications. Moreover, random access channel will have difficulty in handling a large number of short data transmissions from M2M devices, due to a large number of preamble collisions in first handshake step and overload conditions where SINR (signal to interference noise ratio) at eNodeB is violated. One of the key challenges identified by 3 GPP is how to control the overload and congestion of access networks of cellular systems that will be able to support tens of thousands of M2M devices. Designing an effi- 
cient RACH control mechanism will alleviate the overload problem of access network. We propose 2-way handshaking mechanism for M2M traffic rather than 4-way used in conventional LTE while contending to get access to the medium. It reduces the extra overload on RACH.

The conventional 4-way handshaking mechanism was designed to allow concurrent access by $\mathrm{H} 2 \mathrm{H}$ devices, which needs long lasting connection. On the other hand, M2M devices need to send only small amounts of data occasionally. For massive M2M devices going through the full handshaking is not very efficient from the standpoint of bandwidth utilization. For example, after a power outage, all the meters wake up at the same time and tries to send the small data packets simultaneously. These small data packets need to go through a heavy signalling RA procedure and overload the access network. Moreover, using this approach for massive access by M2M devices is risky because the resulting congestion may easily damage both $\mathrm{H} 2 \mathrm{H}$ and $\mathrm{M} 2 \mathrm{M}$ communications [61] and unacceptable performance degradation (high access delay and low success rate).

The number of preambles is limited to 64 per cell coming from one base sequence. Hence, there is a possibility that more than one mobile terminal chooses the same preamble that results in preamble collision. In this thesis, the physical layer of M2M overlay network is implemented with 8 dedicated codes for contention based random access. This leaves 54 codes for other traffic on PRACH which may consist of $\mathrm{H} 2 \mathrm{H}$ traffic and potentially other overlay networks.

VANET usually follows the DSRC (Dedicated Short Range Communications) standard which deploys the IEEE 802.11p standard for wireless communications [76]. IEEE 802.11p works well in urban areas with a dense vehicular population with low speeds and 
small V2V (vehicle to vehicle) and V2R (vehicle to road side unit) distances. However, in suburban, rural areas or highways where the speed limit is higher and the distance between the vehicles and RSUs are larger, IEEE 802.11p is not an optimal solution due to unbounded delay, limited radio range, low scalability issue and lack of persistent infrastructure for V2V communication [7]. To overcome the limitation of VANET V2R communication, in this thesis work we propose to use LTE as a wireless communication medium and to overcome the limitations of IEEE 802.11p in the MAC layer we propose to use CSMA/CA mechanism similar to the one used in IEEE 802.15.4.

The IEEE 802.15.4 is the standard for low data rate, low complexity and low power communication devices. For the applications with high data rate, it is not an optimal solution and it does not support the priority among different traffic classes and suffers from co-existence. In smart city scenarios among different traffic classes, for example, vehicular emergency messages should have higher priority over crowd sensing data. We want to deploy priority among messages. IEEE 802.15.6 supports large data rates and define priorities among different traffic classes through access categories. These features of IEEE 802.15.6 have motivated us to consider this standard to deploy in our proposed priority based CSMA/CA (PM2M) overlay network for smart city application areas. In case of multiple emergency events, the nodes cannot transmit the data within the defined superframe time and may be lost due to overload and collisions. To transmit a high volume of real time data reliably and to control overload in the MAC (medium access control) layer a suitable wireless communication network should be adopted. In this thesis, we propose to use LTE as a wireless backbone network.

In IEEE 802.15.6 during the medium access mechanism a node senses the medium 
through CSMA/CA. If the medium sense busy it freezes the backoff counter and decrement again while medium sense idle again. $\mathrm{H} 2 \mathrm{H}$ traffic is also transmitting in parallel with the M2M traffic therefore, there is a possibility that node might not sense the medium correctly over the backoff period. A Node could hear the noises because of the presence of the physical layer of $\mathrm{H} 2 \mathrm{H}$ traffic. Maybe medium is idle but because of the activity node may sense it as busy also when medium is busy may sense it as idle. There is a possibility of listening medium wrong which could affect the performance of the whole network. We propose an analytical model with considering backoff error and analyze the impact of error on the backoff process.

\subsubsection{Objectives}

In this thesis work we attempt to use ubiquitous coverage of LTE and sharing some resources like preambles in order to have wide coverage area and to create community networks for smart city application areas like transportation, crowd sensing, healthcare data reporting.

In the first part, we use VANET as case study and propose a vehicular machine to machine (VM2M) overlay network over LTE to reduced the limitations of IEEE 802.11p V2R communications in suburban, rural areas or highways where speed limit is higher and distance between the vehicles and RSUs (road side unit) are larger. We propose an analytical model, in order to evaluate feasible combinations of formats and configuration parameters for simultaneous VM2M and $\mathrm{H} 2 \mathrm{H}$ access on $\mathrm{RACH}$. All of the scenarios are

presented in chapter 3. The challenge is to ensure that vehicular M2M traffic (VM2M) and $\mathrm{H} 2 \mathrm{H}$ traffic have fair access to the $\mathrm{RACH}$ resources. We investigated the capacity of 
both subnetworks under various parameters and we have shown that using VM2M overlay network allows fair coexistence of VM2M and $\mathrm{H} 2 \mathrm{H}$ traffic.

In the second part, we propose a priority based machine to machine (PM2M) overlay network over LTE. The objective of this proposed architecture is to ensure that medical emergency real time data or safety messages could transmit to LTE base station with low transfer latency, reliably and according to traffic class priority. We deploy analytical model from [72]. We adopt the model since our proposed architecture's physical layer is shared with H2H LTE communication on PRACH. We modify the adopted proposed model because we have to model the probability of error for our sophisticated MAC layer. The physical layer which is affected by $\mathrm{H} 2 \mathrm{H}$ communications is also affecting the CSMA/CA procedure of medium access control layer. We developed an analytical model with error coming from the physical layer of $\mathrm{H} 2 \mathrm{H}$ traffic, composed of a 3 dimensional Markov chain in order to model the CSMA/CA procedure of IEEE 802.15.6. We investigate the effect of error in our physical and MAC layer with the presence of $\mathrm{H} 2 \mathrm{H}$ traffic. Our approach also provides the ability to calculate the mean number of backoff attempts in every backoff stage. We investigated the capacity of PM2M overlay networks with and without consideration of error and we have shown that overlay network allows fair coexistence of PM2M and $\mathrm{H} 2 \mathrm{H}$ traffic. In order to validate our proposed overlay networks we compare our approaches with a reference approach and shows that overlay network outperforms in terms of access delay and the probability of success. 


\subsubsection{Main contributions}

In this thesis work, we propose M2M overlay network over LTE for wide deployment application areas of smart city. To validate the effectiveness of our proposed architecture we first implement it in VANET scenarios to find the feasible combination of parameters and to find the impact of the capacity of both networks. To deploy the proposed approach in wide deployment scenarios of smart city and define priority among different M2M traffic classes we than implement the overlay network for priority based scenarios. We evaluate the performance of the proposed scheme through a detailed analytical model. Table 1.3 and 1.4 illustrates the main contribution of this thesis work. 
Table 1.3: The illustration of main contributions in this thesis.

\begin{tabular}{|c|c|c|}
\hline Chapter & Contributions & Challenges \\
\hline $\begin{array}{l}\text { Chapter 3: Non priority } \\
\text { CSMA/CA overlay net- } \\
\text { work to support vehicu- } \\
\text { lar traffic over LTE. }\end{array}$ & $\begin{array}{l}\text { 1) Propose a non priority based } \\
\text { CSMA/CA vehicular machine to ma- } \\
\text { chine (VM2M) overlay network over } \\
\text { random access channel (RACH) in LTE } \\
\text { to emulate the control channel (CCH) of } \\
\text { vehicular ad hoc networks (VANETs). } \\
\text { 2) VM2M overlay is implemented over } \\
\text { a dedicated subset of preamble codes, at } \\
\text { the physical layer, medium access con- } \\
\text { trol (MAC) layer is modelled as similar to } \\
\text { IEEE } 802.15 .4 \text { carrier sense multiple ac- } \\
\text { cess (CSMA/CA) mechanism. } \\
\text { 3) It separates the resources between H2H } \\
\text { and M2M at the level of preamble codes. } \\
\text { 4) M2M devices avoid the complexity of } \\
\text { the four-way handshake, which reduces } \\
\text { the interference on the PRACH both to } \\
\text { and from H2H devices, in the same cell } \\
\text { and in neighboring cells. To the best } \\
\text { of our knowledge, this approach has not } \\
\text { been proposed or discussed elsewhere. } \\
\text { 5) We evaluate the performance of the } \\
\text { proposed scheme through a detailed an- } \\
\text { alytical approach. } \\
\text { 6)We analyze the performance of the } \\
\text { scheme through evaluating the interfer- } \\
\text { ence between H2H and M2M traffic in the } \\
\text { form of overload probabilities, and use } \\
\text { these descriptors to dimension the cell ca- } \\
\text { pacity for H2H and M2M users. }\end{array}$ & $\begin{array}{l}\text { Finding the fea- } \\
\text { sible combination } \\
\text { of parameters. }\end{array}$ \\
\hline
\end{tabular}


Table 1.4: The illustration of main contributions in this thesis.

\begin{tabular}{|c|c|c|}
\hline Chapter & Contributions & Challenges \\
\hline $\begin{array}{l}\text { Chapter 4: } \\
\text { Priority- } \\
\text { based } \\
\text { CSMA/CA } \\
\text { overlay } \\
\text { network } \\
\text { over LTE. }\end{array}$ & $\begin{array}{l}\text { 1) Propose a priority based CSMA/CA machine to } \\
\text { machine (PM2M) overlay network over LTE. } \\
\text { 2)The architecture is implemented using a dedicated } \\
\text { subset of preambles at the physical layer, and a car- } \\
\text { rier sense multiple access with collision avoidance } \\
\text { (CSMA/CA) mechanism similar to the one used in } \\
\text { IEEE } 802.15 .6 \text { at the medium access control layer. It } \\
\text { provides the priority among different PM2M traffic } \\
\text { classes and thus ensure the networks quality of ser- } \\
\text { vice. } \\
\text { 3) We consider only two traffic classes (TC) where } \\
\text { traffic class } 0 \text { as lower priority for crowed sensing } \\
\text { data and traffic class } 1 \text { as higher priority for safety } \\
\text { messages or medical emergency data. } \\
\text { 4) Allows M2M terminals to actually transmit data } \\
\text { during PRACH access, which in most cases should } \\
\text { suffice given the short messages typical for M2M } \\
\text { devices, whereas other schemes use random access } \\
\text { to initiate a connection without sending actual data, } \\
\text { which adds to congestion and increases message la- } \\
\text { tency. } \\
\text { 4) The use of overlay network ensures the suitable ac- } \\
\text { commodation of PM2M traffic which are smaller and } \\
\text { have limited computational resources compared with } \\
\text { LTE traffic. } \\
\text { 5) The scheme considers the backoff error during } \\
\text { CSMA/CA mechanism coming from the physical } \\
\text { layer of H2H traffic. } \\
\text { 6) Develop a } 3 \text { dimensional Markov chain in order to } \\
\text { model the backoff procedure of CSMA/CA. } \\
\text { 7) Investigate the effect of error in physical and MAC } \\
\text { layer with the presence of H2H traffic. } \\
\text { 8) Evaluate the performance through a detailed ana- } \\
\text { lytical model and evaluates the impact of error on the } \\
\text { capacity of the overlay network. } \\
\text { 9) Compare the proposed scheme with a reference } \\
\text { scheme to validate the effectiveness of the solution. }\end{array}$ & $\begin{array}{l}\text { Giving priority } \\
\text { among traffic } \\
\text { classes, finding } \\
\text { the impact of } \\
\text { backoff error } \\
\text { and validating } \\
\text { the proposed } \\
\text { approach }\end{array}$ \\
\hline
\end{tabular}


In the first part, we propose a novel $\mathrm{CCH} / \mathrm{SCH}$ architecture over LTE which allows for an efficient implementation of a VANET, with particular attention to areas with large distances and higher vehicle speed. The proposed architecture uses LTE PRACH as the underlying physical layer for a vehicular M2M (VM2M) overlay network. The $\mathrm{CCH}$ architecture is implemented using a dedicated subset of preambles at the physical layer, and a carrier sense multiple access with collision avoidance (CSMA/CA) mechanism similar to the one used in IEEE 802.15.4 low data rate WPAN at the medium access layer. $\mathrm{CCH}$ traffic thus does not need to go through the four way handshake which improves capacity and reduces latency both important traits for safety messages that are routinely passed on $\mathrm{CCH}$. On the other hand, application traffic that uses $\mathrm{SCH}$ still needs to pass the fourway handshake over PRACH in the same way as regular human to human $(\mathrm{H} 2 \mathrm{H})$ access attempts to establish a scheduled communication path. We evaluate three experiments as described below to find out the feasible combinations of parameters.

(a) Firstly, we find out the feasible combinations of preamble formats (PF) and configuration parameters (cf) for simultaneous VM2M and $\mathrm{H} 2 \mathrm{H}$ access on $\mathrm{RACH}$.

(b) Secondly, we have investigated the physical layer limit and capacity of both subnetworks by partitioning the PRACH bandwidth

(c) Thirdly, we evaluate the impact of human to human $(\mathrm{H} 2 \mathrm{H})$ layer over vehicular $\mathrm{M} 2 \mathrm{M}$ (VM2M) overlay when VM2M gives priority to the physical layer to access the base station (eNodeB).

The proposed scheme separates the resources between $\mathrm{H} 2 \mathrm{H}$ and $\mathrm{M} 2 \mathrm{M}$ at the level of preamble codes. Thus, it allows simultaneous $\mathrm{H} 2 \mathrm{H}$ and $\mathrm{M} 2 \mathrm{M}$ access without the need 
for more complex separation schemes, such as the slotted access and access class barring (ACB) proposed in [77].

In the second part, we propose a novel priority based machine to machine(PM2M) overlay network over LTE to transmit a high volume of data reliably and to control overload in the MAC (medium access control) layer. The proposed PM2M architecture overcomes the limitation of IEEE 802.15.4 which does not support priority among different traffic classes. The proposed architecture is same as the VM2M for physical layer with dedicated preambles, whereas, PM2M overlay is now more sophisticated because we have more sophisticated MAC with priorities. In this work we introduce priority differentiation in the overlay network by using the CSMA-CA similar to IEEE 802.15.6 [69] with modifications necessary to match the existing physical layer derived from PRACH.We model the medium access control algorithm including a model of imprecise listening outcome during backoff process, which is shown to decrease the capacity of the overlay network to a non-negligible extent. We demonstrate functionality and performance of our scheme using different schemes and bandwidth of PRACH as well as PRACH design scenarios for micro- and macro cells. Using an accurate characterization of noise and interference caused by other calls from the given cell as well as from the surrounding cells (which is absent from other proposals). We show that the scheme is capable of achieving satisfactory performance as well as sufficient differentiation between traffic classes. It is, thus, suitable for the massive machine-type communications (mMTC) scenario i.e., large number of MTC devices with short messages and low arrival rates which represent one of the major use cases for the development of 5G radio and network technology. Furthermore, our scheme allows M2M terminals to actually transmit data during PRACH access, which in 
most cases should suffice given the short messages typical for M2M devices, whereas other schemes use random access to initiate a connection without sending actual data, which adds to congestion and increases message latency.

We evaluate the capacity and performance of PM2M overlay network over LTE with impact of $\mathrm{H} 2 \mathrm{H}$ layer. Among the 8 different access categories (AC) which indicate the User Priorities (UPs) for accessing the medium, we only consider 2 traffic classes where traffic class (TC) 0 as lower priority nodes and traffic class 5 as higher priority nodes. We propose a backoff error analytical model with 3 dimensional Markov chain in order to model the CSMA/CA procedure of IEEE 802.15.6. Here we evaluated the impact of error in the MAC layer. We have 20 bits and listening of each bit can be wrong from the probability of bit error rate (BER). If all of them or more than half of the bits are wrong we have the wrong listening decision which actually depends on the hardware of the networks. In our case, we define the probability of backoff error as the probability of listening 11 bits are wrong among 20 bits and less than 11 bits are correct. We model a Markov chain with probability of backoff error to investigate the effect of error in our physical and MAC layer with the presence of $\mathrm{H} 2 \mathrm{H}$ traffic. Our approach also provides the ability to calculate the mean number of backoff attempts in every backoff stage. We investigated the capacity of PM2M networks for with and without considering error coming from the physical layer of $\mathrm{H} 2 \mathrm{H}$ traffic and we have shown that overlay network allows fair coexistence of PM2M and $\mathrm{H} 2 \mathrm{H}$ traffic. To validate our approach we compare our without backoff error approach with a reference [87] approach as reference scheme did not consider the presence of error in the backoff process. By performance evaluation, we showed that proposed approach outperforms with respect to the probability of success and mean access delay with the 
presence of $\mathrm{H} 2 \mathrm{H}$ traffic.

\subsection{Thesis organization}

The rest of the thesis is structured as follows:

- Chapter 2 discusses the related research works for LTE based on IEEE 802.15.4 and IEEE 802.15.6.

- In Chapter 3, we have described an non priority overlay network to support vehicular traffic over LTE.

- In Chapter 4 we have described a priority based overlay network over LTE.

- Chapter 5 concludes the thesis and discusses some directions for future work. 


\section{Chapter 2}

\section{Related Work}

\subsection{PRACH overload}

The overload and congestion occur in the random access network (RAN) of LTE during random access channel (RACH) procedure when a massive number of devices tries to get access to the network. The major issue of M2M communications over LTE is the radio resources allocation problem. There are many machines over $\mathrm{H} 2 \mathrm{H}$ traffic and number of preambles are limited. The massive access attempt by M2M devices without increasing the channel capacity overloads the network. There is a possibility of more than one devices use the same preamble and results in a collision. The overload problem affects the network throughput and energy efficiency [6]. Several research studies assert that the random access channel procedure through four step handshake of LTE is not effective for massive $\mathrm{M} 2 \mathrm{M}$ devices to simply transmit one message to the network as may happen in smart city scenarios.

In the past several years a number of techniques to improve performance for M2M 
traffic by alleviating outage due to congestion and overload have been suggested. In [51] the authors propose an efficient power and resource allocation algorithm for relay-assisted M2M communications over LTE. Due to the large distance between M2M devices and eNodeB and poor link condition the M2M devices should employ relays. Their proposed algorithm considers polynomial time complexity at each relay and delays QoS constraint of different $\mathrm{M} 2 \mathrm{M} / \mathrm{H} 2 \mathrm{H}$ traffics as a priority parameter. Through the Numerical results, they showed their proposed design is suitable for delay limited applications with constraints on energy consumption of the system. To control the overload during random access in [22] a dynamic access class barring schemes were proposed. The authors propose a prioritize random access where priority is given to M2M devices by pre-allocating random access channel resources for different M2M classes. The proposed solution is not feasible due to high access delay. In [77] slotted access class barring (ACB) complex schemes were proposed to reduce the congestion by separating the resources among $\mathrm{H} 2 \mathrm{H}$ and M2M devices. In this scheme, each of the MTC devices is associated with a dedicated access slot through its identity and each can perform the RA procedure only through its assigned slots. A dynamic resource allocation scheme was proposed in [47]. The authors propose a self-optimization scheme where eNodeB can automatically increase or decrease the random-access slots for preamble transmission according to traffic load. The authors combine some of the 3GPP techniques of Slotted access, back off and ACB to alleviate the impact of M2M devices over $\mathrm{H} 2 \mathrm{H}$ devices. In [25] the authors propose an optimization scheme to minimize the delay. They propose a dynamic resource allocation scheme for random access slots (RA). They showed the relation between the number of RA slots and delay. The drawback of their proposed scheme is that they didnt consider the lack of available resources for data 
transfer while allocating RA slots for $\mathrm{RACH}$ procedures. However, all of these proposals still rely on the four-way handshake mechanism, which is prone to congestion and overload and, consequently, ill-suited for massive M2M access.

In [68] the authors propose a patch module of LTE PRACH over the default RA module for system level network simulator NS-3. They compare their module with the default one in the respect of access delay. The default simulator considers preamble format 0 and collision in the first handshake step only. The collisions of message 3 were not handled. The authors handled the exception by considering the colliding connection request messages as an error and retransmit the messages until the maximum number of attempts is reached. Through comparison, they showed that the default module shows unrealistic results where all the terminals can successfully complete the RA procedure in less than $1 \mathrm{sec}$ for any number of M2M devices. Whereas, their patch module shows as the number of $\mathrm{M} 2 \mathrm{M}$ increases the access delay is increasing as expected. In [34] the author proposes a novel random-access procedure called two-phase random access procedure (TPRA) to control the overload of LTE PRACH created by a massive number of MTC devices. Through analytical and simulation model they showed that their proposed scheme reduces access delay and improve access probability for MTC devices comparing standard LTE-A RA procedure. TPRA works by separating the massive number of M2M devices into small groups and according to load condition, the base station can assign additional access channel. They mainly focus on decreasing the number of M2M devices which are allowed to access the network simultaneously.

Grouping based uplink resource allocation schemes were proposed in [87]. The authors proposed a grouping based overload control mechanism. To overcome the problem of 
overload and congestion due to concurrent access attempt by massive M2M devices in their proposed approach group leader initiates RA and apply for transmission resources on behalf of the group members. To avoid signalling congestion base station send only one control signal to the group leader. Second group paging scheme is used for the devices who failed to transmit in the current time window. They proposed a five-step handshaking mechanism for RA procedure and also 5 steps mechanism for uplink resource scheduling. The limitations of the proposed approach are, it decreases the number of $\mathrm{M} 2 \mathrm{M}$ devices which are allowed to access the network. The group leader still needs to follow 5 step handshaking mechanism which creates signalling overhead. Selection of group leader among M2M devices is the challenging issue for grouping methods. If the size of the group is massive it can cause the probability of collision high results low access success probability and high access delay. It also has the disadvantages of low resource utilization issue. They evaluate the performance of two traffic classes and compare their results with conventional RA procedures.

\section{Backoff scheme to control overload of PRACH}

In [6] to control the overload in RACH by M2M devices the authors propose a dynamic backoff approach that defines the backoff interval dynamically instead of fixed strategy. They also divided the M2M devices into separate traffic classes to extend the range of possible application areas. Through the network simulator NS-3, they evaluate the performance and show that their scheme controls the impact of $\mathrm{M} 2 \mathrm{M}$ devices over $\mathrm{H} 2 \mathrm{H}$ devices. In [36] the authors propose the strategies to create different backoff classes associated with different M2M traffic. After a collision occurs devices of each class randomly selects backoff interval. It is a fixed backoff approach where the selection of the backoff interval 
depends on the number of classes of devices. In [52] the authors propose a fixed backoff interval approach where each of the devices has fixed backoff time. They differentiate the traffic classes according to backoff time. H2H traffic with low backoff time gets higher priority over M2M with high backoff time. The scheme works well in low traffic scenarios with a low request but it will not be an optimal solution for dense traffic scenarios. However, none of the schemes consider the presence of probability of backoff error during the backoff procedure.

\subsection{Earlier work on overlays in LTE}

\subsubsection{Use of LTE overlay for IEEE 802.15.4 in VANET scenarios}

In order to reach the goal of modern intelligent traffic system in safe, efficient and comfortable way several radio access technologies such as LTE, WiMAX, WiFi e.t.c has been proposed. Here, we consider VANET as a case study and investigate the advantages of using EEE802.15.4 and LTE instead of IEEE 802.11p based VANET scenarios.

IEEE $802.11 \mathrm{p}$ is the standard for vehicular communications, but it is pointed out in [1, $7,12,16]$ that it is not suitable for larger scale scenarios of vehicle to road side unit (V2R) communications due to delay, small radio coverage and low data transmission rate. In [1, 12] a comparative study between VANETs routing protocols was conducted and was shown that all the protocols perform better for urban areas rather than rural areas. The high data rate, low latency and large coverage features of LTE motivated the researchers to consider LTE as an access technology to support the vehicular communication. In [65] an IEEE 802.15.4 based VANET was proposed for urban areas. They showed that IEEE 802.15.4 
low power consumption feature makes it advantages over drawback of IEEE $8802.11 \mathrm{p} \mathrm{high}$ power consumption.

In $[7,31,81]$ a comparative study between IEEE802.11p and LTE was conducted in order to evaluate their suitability for different vehicular applications. Advantages and disadvantages of using LTE instead of IEEE 802.11p are analyzed. As an advantage of using LTE, it was shown that it meets most of the requirements of VANET applications. Simulation studies have shown that LTE supports mobility and provides higher network capacity compared with IEEE $802.11 \mathrm{p}$. However, it was shown in $[2,81]$ that LTE does not show sufficient reliability in terms of safety messages transmitted over PRACH and network can easily become overloaded. The drawback is of using four-way handshaking for small safety messages which limit the capacity and increase latency [61].

Recent studies have shown that [31] LTE meets VANET application requirements by means of delay, scalability, reliability and mobility. However, LTE fails short in scenarios to transmit real safety messages over large coverage areas. It is also challenging to meet all the requirements in the presence of higher cellular network traffic load. Therefore, a careful examination is needed of the LTE architecture and the manner in which it can be used to support requirements for $\mathrm{CCH}$ in a VANET.

In a dense vehicle environment, vehicular ad hoc networks (VANETs) cannot meet the requirements of safety messages. IEEE $802.11 \mathrm{p}$ protocol suffers from a high rate of collisions while transmitting safety messages in a high dense environment as its contention window (CW) cannot be adjusted with vehicles density. In [3] the authors proposed an enhanced mechanism that decreases the failure rate of safety messages. Their proposed scheme uses slotted ALOHA reservation method where each node will be assigned a num- 
ber of slots for transmission. Although they showed that their scheme decreases failure rate, the use of ALOHA still suffers from being prone to collisions and CSMA always outperforms slotted ALOHA [14]. In [83, 85, 89] the authors proposed dynamic contention window mechanism to decrease the failure of safety message, where $\mathrm{CW}$ values depend on the vehicles density. However, all of these mechanisms still suffer from packets collision and increases access delay at high vehicle density environment.

LTE is suitable over IEEE 802.11p based VANET for transferring safety messages was shown through an analytical and simulation model in $[8,46]$. In VANET depending on the distance between RSU and vehicles different transmission range is used [16]. To transmit real-time safety messages we need continuous access with high data transmission rates and large coverage area which is the features of LTE.

A hybrid architecture of combining IEEE 802.11p and LTE was proposed in $[27,79]$. Use of sole LTE was not proposed due to high communication cost between the vehicles and base stations and hand-off load at the base stations. It was shown that the integrated architecture improves throughput, minimize delay and packet drop rate.

The work in [64] includes a comparison between infrastructure-based wireless technologies: IEEE 802.11 and WiMAX in V2I communications. They study the limitation and feasibility of using both technologies for varying data rates, vehicle speeds and network deployments. In [5] it was showed that WiMAX`s signal interference is high compared with LTE.

In a nutshell, the use of four-way handshaking procedure of LTE for massive network access in control channel $(\mathrm{CCH})$ is inefficient and leads to network overload. This has motivated our research efforts towards vehicular machine to machine (VM2M) architecture, 
where $\mathrm{CCH}$ is implemented as an overlay in PRACH to transmit safety messages.

\subsubsection{Use of LTE overlay for priority based networks}

IEEE 802.15.6 supports priority schemes whereas IEEE 802.15.4 does not support priority among different traffic classes. Before introducing the IEEE 802.15.6 standard, IEEE 802.15.4/Zigbee and Bluetooth were considered as the efficient physical and MAC layer protocols for application scenarios of healthcare. Existing networks proposed in $[10,13,19,30,33,50,78]$ employ Zigbee/IEEE 802.15 .4 as communication protocol, are optimized for short-range communication networks. IEEE 802.15.4 standard's low power consumption, short message transmission and short communication range made it advantageous to employ over other technologies. Some of the researchers employ Bluetooth $[37,38,66]$ to implement IEEE 802.15.6/WBAN. Bluetooth does not support automatic network formation and it is a very slow process. Low power consumption is the most important and challenging requirements for the system whereas, Bluetooth 's power consumption is higher than IEEE 802.15.4 and it uses power to maintain a connection which is the drawback and inefficient for IEEE 802.15.6 communications [88]. In [75] the performance of IEEE 802.15.4 based and Bluetooth based IEEE 802.15.6/WBAN are compared with experiments. Their results show that neither of them has significant advantages over another as Zigbee overload the network in presence of high density of node and Bluetooth consumes more power.

In [29] the authors did a comparative study between IEEE 802.15.4 and IEEE 802.15.6. They showed that to transmit emergency medical records reliably, IEEE 802.15.4 does not satisfy the requirements because it does not support high data rate applications. In [90] 
the authors compare the IEEE 802.15.6 and IEEE 802.15.4 based MAC protocol for medical health care system in terms of energy consumption, number of used nodes and sensors data rates. Through simulation, they showed that the choice of the protocol depends on the medical sensors data rate. They evaluate that for sensors like temperature and glucose which requires minimum data rate (less than $40 \mathrm{~kb} / \mathrm{s}$ ) use of IEEE 802.15 .4 is more beneficial. However, for the sensors like ECG and Endoscopy which requires high data rate, the use of IEEE 802.15.6 is more beneficial. They also showed that in terms of throughput and reliability IEEE 802.15.6 results in a good performance with high data rate although sacrifices energy consumption.

Integration of Zigbee with WLAN, WAN, WiMAX and LTE was proposed in [17, $33,39,40,43]$ to enlarge the radio coverage of IEEE 802.15.6. In [18] the authors proposed a 3 tier architecture e-health systems, where tier 1 is the communication between the user and radio coverage of about 2 meters. Sensors are deployed on the human body or planted inside the body or can be attached in the human clothes. The sensors send their data to a personal portable server (PS). Tier 2 is named as inter-BSN (Body sensor network) communication, the communication between PS to one or more access points (AP). The functionality of tier 2 is to interconnect the BSNs with internet and cellular networks. Tier 3 is, beyond BSN communication which helps to access the patients information remotely through cellular network or internet. It enhances the limitation of radio coverage and forwards the e-health systems one step further. The authors of [33] deploy the architecture of [18] and propose the scenarios of IEEE 802.15.6 integration with WAN, WiMax and LTE. They demonstrate their topology works fine for all 3 types of ZigBee topology networks. Through the simulation, they showed that Zigbee end devices can successfully transmit 
data in real to other networks. There is a problem of radio interference and coexistence issue between IEEE 802.15.4 based IEEE 802.15.6/WBAN and WLAN networks as most of the channels of WLAN is overlapped by IEEE 802.15.4. A fault tolerant architecture was present in [28] as a smart city scenario, where two communication protocols are used for data transmission, low power IEEE 802.11n (WiFi) and LTE. In this system, WiFi is used for short-range communication as it is widely used protocol for home networks while LTE is used for long-range communications to extend the radio coverage. As a fault tolerance mechanism, they proposed that, if any link fails the data can be transmitted using other paths to send data from sensor to the controller and for response received by the actuators. However, they didnt consider the co-existence issue of both technologies.

In [69] the author studied the performance of a healthcare system through interconnecting IEEE 802.15.6 with IEEE 802.11e based WLAN for transferring the data between a hub and the medical information system. The author evaluates the performance through analytical and simulation models. Their proposed architecture uses RTS/CTS mechanism for accessing the medium in the WLAN which causes overload in the network and coexistence issue. The author also did not consider the presence of backoff error while accessing the medium to transmit the packets. The authors in [60] evaluate the performance of IEEE 802.15.6 bridges with IEEE 802.11 based WLAN. They did not consider the user priorities while transmitting messages.

In [33] it was shown that there is no interference when Wimax or LTE integrate with IEEE 802.15.6 network. However, at high peak data rate WiMax results in high end to end delay than LTE. It was proposed in [17] that LTE supports reliable data transmission with high data rates for real-time safety messages over large coverage areas. To enlarge the radio 
coverage they integrate IEEE 802.15.6 with LTE because they showed that existing architecture is not suitable for the scenarios with high mobility due to the fluctuation of wireless links. Furthermore, when the amount of content is large, the quality of delivery is hard to be guaranteed. Most of the existing scenarios are focus on low mobility and bandwidth so it is not good for high mobility scenarios. The authors deploy a real-time highly movable rescue scenarios and justify that LTE provides high data rate communications between rescuers.

Recently, an overlay network for M2M traffic was proposed that dedicates a portion of PRACH resources to M2M traffic [42]. The overlay network is based on CSMA-CA mechanism similar to IEEE 802.15.4 [59] and allows M2M access to be completed without the four-way handshake. $\mathrm{H} 2 \mathrm{H}$ traffic concurrently uses remaining resources and is coupled with M2M traffic through the SINR at the eNodeB. Further differentiation is possible using different and explicit power levels for $\mathrm{M} 2 \mathrm{M}$ and $\mathrm{H} 2 \mathrm{H}$ traffic [41]. However this approach $[41,42]$ does not address two important aspects of M2M communications. First, differentiation among different traffic classes in the M2M overlay network is needed. Second, SINR coupling between $\mathrm{M} 2 \mathrm{M}$ and $\mathrm{H} 2 \mathrm{H}$ traffic classes has been considered only at the packet reception level but not at the level of listening to the medium during the CSMA-CA backoff process. This could produce incorrect durations of backoff process and a too conservative estimate of the congestion at the overlay network.

The features of LTE's higher volume data transmission rate in timely fashion and to deploy proposed architecture in wide deployment scenarios for different M2M traffic classes, motivated us to use LTE with PM2M communications to enlarge the radio coverage. 


\subsection{Chapter summary}

In this chapter, we have surveyed the current research works on the overload and congestion control for PRACH of LTE. Most of the current solutions consider conventional 4-way handshaking and slotted ALOHA as medium access protocol which is prone to congestion and causes a heavy collision in the network. There is a need for a new approach to controlling the overload. To analyze and propose a new approach we investigate the current proposed approaches limitations in this chapter.

To investigate the proposed schemes effectiveness we deploy it in smart city application area like smart transportation system (VANET). We surveyed the limitations of current VANET standard and propose the possible solution to overcome the limitations in chapter 3.

To give priority among different applications of smart city scenarios and for wide deployment we investigate the priority based networks usefulness with LTE. The current research works did not consider the backoff error during the backoff procedure. We propose a model considering backoff error in chapter 4 . We compare our proposed approach with proposed research work [87] as they also consider M2M priority traffic classes. 


\section{Chapter 3}

\section{Non priority CSMA/CA overlay network}

\section{to support vehicular traffic over LTE}

In this chapter, we consider VANET as a case study for smart city application areas. We propose a non-priority vehicular machine to machine (VM2M) overlay network over random access channel $(\mathrm{RACH})$ in LTE that aims to emulate the control channel $(\mathrm{CCH})$ of vehicular ad-hoc networks (VANETs). VM2M overlay is implemented over a dedicated subset of preamble codes, at the physical layer, and uses a medium access control (MAC) layer modelled as IEEE 802.15.4 carrier sense multiple access (CSMA/CA) mechanism. We evaluate the performance and interaction of regular LTE $(\mathrm{H} 2 \mathrm{H})$ traffic and VM2M traffic, in particular, the impact of RACH resource configuration and preamble format (PF) in large cells. We evaluate the impact on the performance of VM2M and $\mathrm{H} 2 \mathrm{H}$ traffics for various scenarios like priority, preamble format and traffic condition. The results show that VM2M overlay network allows fair coexistence of VM2M and $\mathrm{H} 2 \mathrm{H}$ traffic. 


\subsection{Introduction}

Vehicular Ad hoc network (VANET) supports communication between vehicle to vehicle (V2V) and vehicle to roadside unit (V2R) to transfer safety messages through the dedicated control channel $(\mathrm{CCH})$ and other messages like infotainment, telematics through one or more service channels (SCH). IEEE 802.11p standard for VANETs enables V2V and V2R communications through the onboard unit (OBUs) which is vehicles interface to VANET can also be considered as a vehicular machine to machine (VM2M) device to take part in vehicular communications. However, the standard is not well suited for VM2M communications due to unbounded delay, limited radio range, low scalability, and lack of persistent infrastructure [7]. It has been shown to be prone to congestion, early saturation and rapid decrease of transmission rate under a high vehicle density, large RSU coverage area and large distances between RSUs and OBUs [58].

A better solution would be to use Long-Term Evolution (LTE), a widely used standard for $4 \mathrm{G}$ cellular communications. Its high data rate, low latency and large coverage features make it attractive for VM2M deployment in suburban and rural areas, as well as on highways. Recent studies have shown that LTE can easily meet VANET application requirements in terms of delay, scalability, reliability and mobility [31]. However, LTE still falls short in scenarios where safety messages with high data rate need to be reliably transmitted over large coverage areas. Therefore, a careful examination is needed of the LTE architecture and the manner in which it can be used to support requirements for $\mathrm{CCH}$ in a VANET.

In this chapter, we propose a novel non-priority based CSMA/CA, $\mathrm{CCH} / \mathrm{SCH}$ architecture over LTE which allows for an efficient implementation of a VANET, with particular 
attention to areas with large distances and higher vehicle speed. The proposed architecture uses LTE PRACH as the underlying physical layer for a vehicular M2M (VM2M) overlay network. The $\mathrm{CCH}$ architecture is implemented using a dedicated subset of preambles at the physical layer, and a carrier sense multiple access with collision avoidance (CSMA/CA) mechanism similar to the one used in IEEE 802.15.4 low data rate WPAN at the medium access control layer. $\mathrm{CCH}$ traffic thus does not need to go through the four-way handshake which improves capacity and reduces latency both important traits for safety messages that are routinely passed on $\mathrm{CCH}$. On the other hand, application traffic that uses $\mathrm{SCH}$ still needs to pass the four-way handshake over PRACH in the same way as regular human to human $(\mathrm{H} 2 \mathrm{H})$ access attempts to establish a scheduled communication path. The challenge is, then, to ensure that vehicular M2M traffic (VM2M) and $\mathrm{H} 2 \mathrm{H}$ traffic both have fair access

to the PRACH resources. We investigate the capacity of both sub-networks in a range of parameter values, priority, preamble format, bandwidth and show that the VM2M overlay network allows fair coexistence of VM2M and H2H traffic.

\subsection{LTE-based VM2M overlay architecture}

We will now describe the architecture of the proposed LTE based overlay network that supports V2R communications. We distinguish between SCH which uses regular LTE access and $\mathrm{CCH}$ which uses the VM2M overlay, as we explain in the following. Depending on the cell size, vehicle speeds and traffic condition VM2M can be implemented using different preamble formats $(\mathrm{PF})$ and configuration index $\left(c_{f}\right)$. Figure 3.1 shows the proposed VM2M overlay superframe with $\mathrm{PF}=2$. 


\subsubsection{Service channel(SCH)}

SCH traffic uses regular LTE access where a mobile terminal (MT, in this case, an $\mathrm{OBU})$ that has no allocated radio resources must first perform random access to connect to the network. Random access can be contention-based, in case of a new connection, or contention-free; in the former case, the standard-prescribed four step handshaking mechanism is used as described in chapter 1.

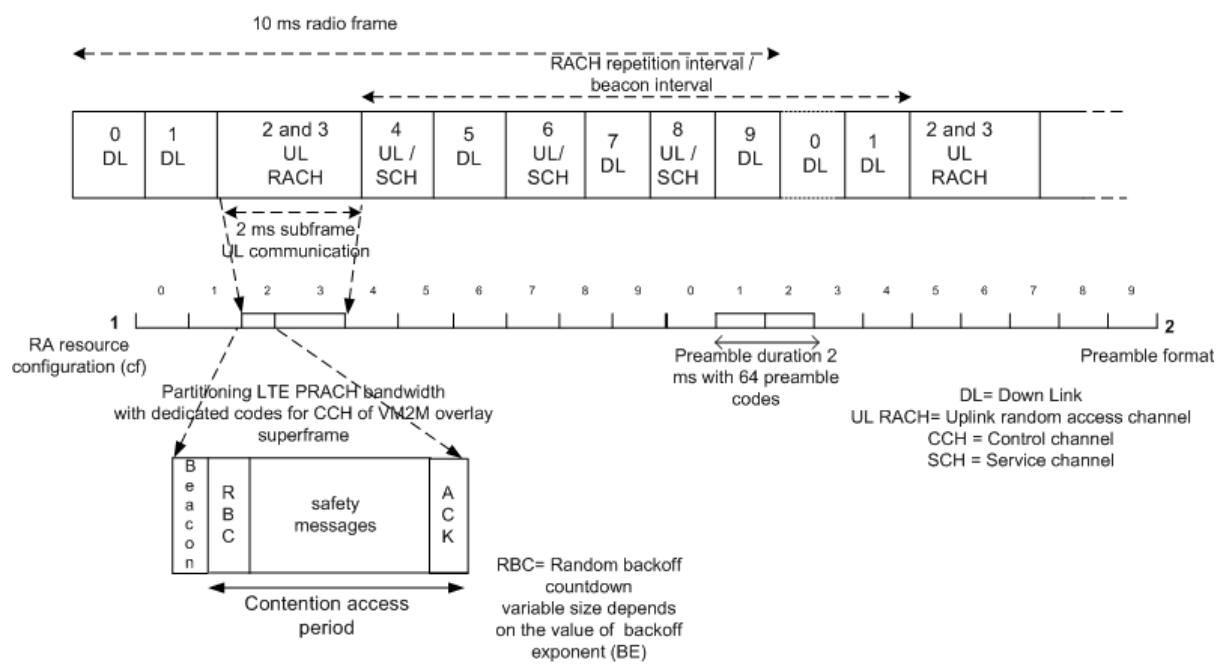

Figure 3.1: LTE and VM2M Overlay Superframe with PF 2 in TDD Mode (2 ms Subframe).

\subsubsection{VM2M overlay for control channel $(\mathrm{CCH})$}

In our approach, $\mathrm{CCH}$ is allowed to share the PRACH with regular (SCH) traffic. However, random access on PRACH can fail due to the following two mechanisms-

First, as the number of preambles is limited (the default number is 64 per cell), a collision may occur when two or more MTs select the same preamble for initial access $[48,91]$. Collided preambles are re-transmitted after a random backoff time that spreads out access 
to maximize the probability of success [21].

Second, congestion can occur on account of noise and interference generated by other nodes, both in the same cell and in neighbouring cells, as other logical channels may use some of the resources of the PRACH in the current cell. It may occur in both the first and third steps of the handshake. However, in absence of congestion, eNodeB might be able to decode a preamble even upon a collision and subsequently grant access to one of the terminals; this is known as capture effect.

Congestion was shown to be a much bigger problem than collisions [61], partly due to the fact that the four-step handshake is effectively an overkill for $\mathrm{CCH}$ messages which are short and occur in random bursts [11]. We note that one of the key challenges identified by 3GPP is how to control the overload and congestion in case of simultaneous access by tens of thousands of M2M devices [77]. Random access could be made more efficient if safety messages on the $\mathrm{CCH}$ could be decoded without requiring the terminal to go through the complete handshake.

We propose to implement $\mathrm{CCH}$ in the following manner. At the physical level, a total of $N_{M}$ preambles is reserved for $\mathrm{CCH}$; this number need not be hypothetically, 8 or 10 preambles out of 64 would sufficeas the aggregate traffic volume on $\mathrm{CCH}$ is much lower than that on $\mathrm{SCH}$. Reserving the preambles for $\mathrm{CCH}$ use will accomplish resource separation at the preamble level and reduce the potential for collisions with $\mathrm{SCH}$ messages. The remaining preambles will be used for new and handover connections, and potentially for other overlay networks as well. Once the connection is established, $\mathrm{SCH}$ traffic such as infotainment and vehicular telematics can use any other scheduled channel available in LTE.

Data bits of a $\mathrm{CCH}$ message will be multiplexed over the reserved preambles. In addi- 
tion, preamble elements used as a chipping sequence for a single data bit; this will improve the SINR for the overlay because of the detection mechanism. Namely, SINR for detecting a regular (i.e., $\mathrm{H} 2 \mathrm{H}$ or $\mathrm{SCH}$ ) preamble is based on the entire preamble duration. On the other hand, the SINR threshold for detecting a preamble in the overlay must hold for each bit in the preamble. As the result, the latter SINR is higher than the former.

At the MAC level, the preambles reserved for $\mathrm{CCH}$ are used to implement a slotted CSMA-CA MAC protocol similar to IEEE 802.15.4 [59]. Assuming that one backoff slot has 20 sequence elements, we obtain the unit backoff time as $t_{b o f f}=20 / R=18.51 \mu \mathrm{s}$, which is close to the value of $20 \mu$ s used in IEEE 802.11 at the raw data rate of $1 \mathrm{Mbps}$. Time for preamble transmission (typically, $0.8 \mathrm{~ms}$ ) becomes the superframe time for VM2M overlay network. The whole LTE frame duration is 540 overlay backoff periods. The time interval between two active periods/ superframe is the distance between two beacons, i.e., the period between two PRACH subframes. It depends on the PRACH configuration parameter $c_{f}$, i.e., on the number of PRACH resources available in the LTE frame time and calculated as $\left\lfloor P B=540 / c_{f}\right\rfloor$. The active portion of the superframe, then, has the size of $N_{Z C} N_{M} / 8 N_{b}$ bytes in the superframe; the guard time and cyclic prefix may be understood as the superframe inactive time [17]. Note that this is conceptually different from the $B O$ and $S O$ parameters that regulate active superframe size and distance between consecutive beacons in the original IEEE 802.15.4 standard [59]. The superframe will begin immediately after the reception of beacon and after completing the random backoff, the terminal will transmit the message to eNodeB. eNodeB will acknowledge a successfully decoded message. Non-acknowledged messages will be retransmitted until successful or until the retransmission limit is reached. When the $\mathrm{CCH}$ queue is found to contain a packet to trans- 
mit, the terminal (i.e., the OBU) synchronizes with the beacon and begins the CSMA-CA transmission algorithm. It picks a random backoff value, counts down to zero (decrementing the backoff value at the boundary of the current backoff period), and checks whether the medium is busy in two successive backoff slots. If it finds that the medium is busy, the terminal initiates a new backoff countdown. If not, it transmits the packet using the preamble sequence in the manner described above. Also, if the current superframe does not have enough time to finish the countdown, the node needs to wait until the next superframe active period.

In this manner, $\mathrm{CCH}$ traffic (typically safety messages) can be sent quickly without going through the four-step handshake, while $\mathrm{SCH}$ traffic can go the regular route, first by creating a connection through PRACH and then using other LTE scheduled channels for actual content.

\subsubsection{VM2M random access procedure}

We propose a two way handshaking mechanism to control the overload situation of the RA procedure where M2M devices only use the dedicated preambles to send their data packets. This scheme allows M2M terminals to actually transmit data during PRACH access, which in most cases should suffice given the short messages typical for M2M devices, whereas other schemes use random access to initiate a connection without sending actual data, which adds to congestion and increases message latency. We show that the scheme is capable of achieving satisfactory performance as well as sufficient differentiation between traffic classes. It is, thus, suitable for the massive machine-type communications (MTC) scenario i.e., large number of MTC devices with short messages and low arrival 
rates. Furthermore, our scheme allows M2M terminals to actually transmit data during PRACH access, which in most cases should suffice given the short messages typical for M2M devices, whereas other schemes use random access to initiate a connection without sending actual data, which adds to congestion and increases message latency.

Step 1: In the first step the M2M devices send its data by using the available dedicated preambles. As we separate the resources between $\mathrm{H} 2 \mathrm{H}$ and M2M, M2M devices will not use other preambles allocated for $\mathrm{H} 2 \mathrm{H}$ traffic. M2M devices will compete to get access to PRACH following CSMA/CA mechanism like the one used in IEEE 802.15.4. and in parallel with $\mathrm{H} 2 \mathrm{H}$.

Step 2: After transmitting the data packet the M2M device will wait for the acknowledgement. If it does not receive the acknowledgement due to collision it will retransmit until the maximum retransmission limits.

\subsubsection{H2H overload probability during random access handshake}

$\mathrm{H} 2 \mathrm{H}$ terminals/traffic are attempting random access occasionally and infrequently with $\mathrm{H} 2 \mathrm{H}$ request arrival rate of Poisson distribution. The mean arrival rate $\lambda_{w}$ of $\mathrm{H} 2 \mathrm{H}$ traffic can be calculated for a single PRACH resource as, $\lambda_{W H}=\left(\lambda_{w} T_{f}\right) / c_{f}$ where, $T_{f}$ is the LTE superframe time and $c_{f}$ determines the number of PRACH resources per LTE frame. LTE PRACH is overloaded by preamble collision and external interference caused by the surrounding networks like VANET. To detect a signal successfully at eNodeB of LTE the error rate should be low and signal to interference noise ratio (SINR) should not be under the certain threshold value. 
In the first and third step of 4-way handshaking mechanism, LTE results in excessive uplink transmission and violets the SINR threshold and cause overload on PRACH. H2H networks SINR (in decibels) is the ratio of preamble sequence energy $\left(E_{\text {seq }}\right)$ over noise power density $\left(N_{0}\right)$ for $n$ number of $\mathrm{H} 2 \mathrm{H}$ traffic including initial and handoff requests and all the transmissions and receiving are done with same power level. SINR for $\mathrm{H} 2 \mathrm{H}$ is

$$
\begin{aligned}
E_{s e q} / N_{0} & =\frac{\frac{P_{s e q} I_{1}}{R}}{\frac{(n-1) I_{1}+N_{M} I_{1}+\eta_{p, 1}+\eta_{0} W}{W}} \\
& =P_{s e q} \frac{W / R}{n-1+N_{M}+\eta_{p, 1} / I_{1}+\eta_{0} W / I_{1}}
\end{aligned}
$$

where,

$P_{s e q}=$ preamble sequence length in bits

$\mathrm{W}=$ LTE bandwidth

$\mathrm{R}=\mathrm{LTE}$ PRACH preamble data rate

$I_{1}=$ Received signal power

$\eta_{p, 1}=$ outer cell interference power

$\eta_{0}=$ spectral density of white noise

$N_{M}=\mathrm{VM} 2 \mathrm{M}$ preamble code which are always active

Detection of the successful preamble over the preamble sequences depends on the ratio of $E_{\text {seq }} / N_{0}$. SINR of preamble detection is $10 \log \frac{E_{\text {seq }}}{N_{0}}$ for VM2M traffic. Threshold value of $18 d B$ (or $-11.23 d B$ ) guarantees that probability of preamble missed detection is smaller than $10^{-2}$ and probability of false alarm is less than $10^{-3}$ for eNodeB. The ratio of the outer cell interference and received signal power $\eta_{p, 1} / I_{1}$ of PRACH is a Gaussian distribution with mean and variance denoted by $k_{m, 1}$ and $k_{v, 1}$ [61]. In case of threshold exceed the 
overload condition occurs and preamble can not be detected successfully. The probability of PRACH overload in the first handshake step is,

$$
O \_P R A C H_{n}=P_{r}\left(n+\frac{\eta_{p, 1}}{I_{1}}>\frac{W}{R T_{1}}-N_{M}-\frac{W \eta_{0}}{I_{1}}\right)
$$

The PRACH overload for third handshake step for $j$ number of $\mathrm{H} 2 \mathrm{H}$ traffic is,

$$
Q_{-} P R A C H_{j}=P_{r}\left(j+\frac{\eta_{p, 3}}{I_{3}}>\frac{W_{3}}{R_{3} T_{3}}-\frac{W_{3} \eta_{0}}{I_{3}}\right)
$$

The third step also carries important information and we assume that the spectral effi-

ciency of uplink transmission is as same as first step, $\frac{W_{3}}{R_{3}}=\frac{W}{R}$. This steps threshold value is $-5 \mathrm{~dB}$ because it is observed over a single bit. The ratio of the outer cell interference and received signal power $\frac{\eta_{p, 3}}{I_{3}}$ of PRACH is a Gaussian distribution with mean and variance denoted by $k_{m, 3}$ and $k_{v, 3}[61]$

\section{H2H PRACH overload during preamble collision}

LTE PRACH is overloaded by preamble collision. To calculate the PRACH overload we need to know the total Poisson arrival rate of per PRACH for all the traffic including new, returning and handover calls, $\lambda_{\text {total }}=\lambda+\lambda_{p c 1}+\lambda_{p c 3}+\lambda_{h}$. Now we need to find the probability of $n$ arrivals of neither new or handover $\mathrm{H} 2 \mathrm{H}$ calls is,

$$
P_{n}=\frac{\left(\lambda_{\text {total }}\right)^{n}}{n !} e^{-\lambda_{\text {total }}}
$$

For $n \mathrm{H} 2 \mathrm{H}$ access attempts in PRACH resources, from overload equation of first handshake step 3.2 we obtain the overload probability as, 


$$
O_{-} P R A C H_{n}=\operatorname{erfc}\left(\frac{\frac{W}{R} \frac{1}{T_{1}}-\frac{W \eta_{0}}{I_{1}}-n+1-N_{M}-k_{m, 1}}{\sqrt{k_{v, 1}}}\right)
$$

where erfc is a complementary error function,

$$
\operatorname{erfc}(x)=\frac{2}{\sqrt{(\Pi)}} \int_{x}^{\infty} e^{-\frac{t^{2}}{2}} d t
$$

Thus the total overload probability for first handshake step due to collision and interference is obtain by averaging the $\mathrm{H} 2 \mathrm{H}$ load:

$$
P_{O, 1}=\sum_{n=2}^{\infty} P_{n} O_{-} P R A C H_{n}
$$

by using euation 3.4 and 3.5 ,

$$
=\sum_{n=2}^{n_{\max }} \frac{\left(\lambda_{\text {total }}\right)^{n}}{n !} e^{-\lambda_{\text {total }}} O_{-} P R A C H_{n}
$$

For the third handshake step collision probability that $\mathrm{H} 2 \mathrm{H}$ terminals collide when $j>1$ is,

$$
P_{3}(j)=e^{-\lambda_{i}, L_{3}} \frac{\lambda_{i, L_{3}}^{j}}{j !}
$$

where,

$$
\lambda_{i, L_{3}}=\text { arrival rate of third step L2/L3 messages }
$$

Now to find the total overload of PRACH for third handshake step the overload probability of equation 3.3 can be written with complementary error function as equation 3.6 with different values of bandwidth, spectral efficiency and SINR. 


$$
Q_{-} P R A C H_{j}=\operatorname{erfc}\left(\frac{\frac{W_{3}}{R_{3}} \frac{1}{T_{3}}-\frac{W_{3} \eta_{0}}{I_{3}}-j-k_{m, 3}}{\sqrt{k_{v, 3}}}\right)
$$

Thus the overall PRACH overload probability for third handshake step with preamble collision and inter cell interference is,

$$
P_{Q}=\frac{\sum_{j=2}^{j} P_{3}(j) Q_{-} P R A C H_{j}}{\sum_{j=2}^{j} P_{3}(j)}
$$

\subsubsection{VM2M overload calculation}

Bit error rate (BER) of overlay VM2M depends on the $\mathrm{H} 2 \mathrm{H}$ traffic of the PRACH. Through the BER, VM2M knows the presence of $\mathrm{H} 2 \mathrm{H}$. To estimate the BER of VM2M traffic we need to find the overload probability of VM2M based on SINR requirements for PRACH. SINR of VM2M traffic is the ratio of bit energy $\left(E_{b}\right)$ and noise spectral power $\operatorname{density}\left(N_{0}\right)$

$$
\begin{aligned}
E_{b} / N_{0} & =\frac{\frac{N_{b} I_{1}}{R}}{\frac{(n) I_{1}+\left(N_{M}-1\right) I_{1}+\eta_{p, 1}+\eta_{0} W}{W}} \\
& =N_{b} \frac{W / R}{n+N_{M}-1+\eta_{p, 1} / I_{1}+\eta_{0} W / I_{1}}
\end{aligned}
$$

where,

$N_{b}=$ preamble elements

$\mathrm{W}=\mathrm{LTE}$ bandwidth

$\mathrm{R}=\mathrm{LTE}$ PRACH preamble data rate

$I_{1}=$ Received signal power 
$\eta_{p, 1}=$ outer cell interference power

$\eta_{0}=$ spectral density of white noise

$N_{M}=$ VM2M preamble code which are always active

The overload probability of VM2M data on each preamble is depends on the $\mathrm{H} 2 \mathrm{H}$ traffic and inter cell interference. It can be derived as a same way which is analogous to equations 3.5 and 3.2

$$
\begin{gathered}
M_{\_} \text {oload }_{n}=P_{r}\left(n+\frac{\eta_{p, 1}}{I_{1}}>\frac{W N_{b}}{R T_{M}}-N_{M}-\frac{W \eta_{0}}{I_{1}}\right) \\
=\operatorname{erfc}\left(\frac{\frac{W N_{b}}{R} \frac{1}{T_{M}}-\frac{W \eta_{0}}{I_{1}}-n-N_{M}+1-k_{m, 1}}{\sqrt{k_{v, 1}}}\right)
\end{gathered}
$$

The total VM2M overload probability can be obtain as,

$$
P_{M \_o l o a d}=\sum_{n=0}^{\infty} P_{n} M_{-} \text {oload }{ }_{n}
$$

by using equation 3.13 and 3.4 ,

$$
=\sum_{n=2}^{n_{\text {max }}} \frac{\left(\lambda_{\text {total }}\right)^{n}}{n !} e^{-\lambda_{\text {total }}} M_{\text {_oload }}
$$

using the VM2M overload probability we could approximate the BER as half of the overload.

$$
B E R_{V M 2 M}=0.5 P_{M \_o l o a d}
$$




\subsection{Performance of $\mathrm{VM} 2 \mathrm{M}$ in the presence of $\mathrm{H} 2 \mathrm{H}$ traffic}

In the numerical evaluation, we evaluate the performance of the analytical model in order to evaluate feasible combinations of preamble formats and configuration parameters for simultaneous VM2M and $\mathrm{H} 2 \mathrm{H}$ access on $\mathrm{RACH}$. We evaluate the performance of following three experiments:

(a) Combinations of preamble formats (PF) and configuration parameters (cf) for simultaneous VM2M and $\mathrm{H} 2 \mathrm{H}$ access on $\mathrm{RACH}$.

(b) PRACH bandwidth partitioning for VM2M

(c) Priority based VM2M communications over LTE

The challenge is to ensure that vehicular M2M traffic (VM2M) and $\mathrm{H} 2 \mathrm{H}$ traffic have fair access to the RACH resources. We investigated capacity of both subnetworks under various parameters and we have shown that using VM2M overlay network allows fair coexistence of VM2M and $\mathrm{H} 2 \mathrm{H}$ traffic.

We assume that cell has a total of $N=N_{h}+N_{i}+N_{M}=64$ orthogonal ZC preambles with $N_{h}=10$ allocated to handoff calls, $N_{i}=46$ allocated to $\mathrm{H} 2 \mathrm{H}$ traffic in contention mode, and $N_{M}=8$ allocated to M2M overlay traffic. LTE system bandwidth is $5 \mathrm{MHz}$ and PRACH bandwidth is $\mathrm{W}=1.08 \mathrm{MHz}$. Preamble length is set to $N_{Z C}=839$ elements $[26,74]$. The physical layer of VM2M overlay network is implemented with 8 dedicated codes for contention based random access. This leaves 54 codes for other traffic on PRACH which may consist of $\mathrm{H} 2 \mathrm{H}$ traffic and potentially other overlay networks. Data bits of overlay network are multiplexed over the $N_{M}$ sequences. In order to maintain acceptable SINR, $N_{b}$ preamble elements are used for each data bit. $\mathrm{H} 2 \mathrm{H}$ preamble is detected 
based on SINR for the whole preamble duration while in order to detect the M2M data bits high SINR is needed. M2M codes are bundled together such that each code carries $1 / N_{M}$ of users data bits. VM2M physical layer data rate depends on the $\mathrm{H} 2 \mathrm{H}$ physical data rate $\mathrm{R}$ and $N_{b}$, as $R_{M}=R N_{M} / N_{b}$. The VM2M devices follow the IEEE 802.15.4 slotted CSMA/CA algorithm to access to the network. The algorithm starts when the ready queue holds at least a packet to transmit and gets a beacon message from the eNodeB. In case of ongoing transmission the nodes need to backoff and wait until the end of the transmission. The backoff value is decremented at the boundaries of the backoff period. The algorithm locates the boundary of the next backoff period and all operations must be synchronized with the backoff periods. If the current superframe does not have enough time to finish the countdown the node needs to wait until the next superframe active period. The whole LTE frame duration is 540 overlay backoff periods. The duration between two active periods/ superframe is time period between two beacons or between two RACH subframes. It depends on the $\mathrm{RACH}$ configuration parameter $c_{f}$ i.e. on the number of $\mathrm{RACH}$ resources available in the LTE frame time and calculated as $\lfloor P B=540 / c f\rfloor$. In our work we experiment with different combinations of $\mathrm{PF}, c_{f}$, preamble codes and $\mathrm{H} 2 \mathrm{H}$ traffic to find a feasible combination for our overlay networks. We consider $c_{f}=1$ to 5 PRACH resources respectively and $\mathrm{PF}=0$ and 2 . Table 3.1 shows the parameters with the values we use in our experiments. 
Table 3.1: Implementation Parameters of VM2M overlay Network

\begin{tabular}{|l|l|}
\hline codes per cell, N & 64 \\
codes for H2H traffic, $N_{i}$ & 46 \\
codes for VM2M overlay traffic, $N_{M}$ & 8 \\
LTE frame duration & 540 overlay backoff periods \\
LTE system bandwidth & $5 \mathrm{MHz}$ \\
PRACH bandwidth & $\mathrm{W}=1.08 \mathrm{MHz}$ \\
preamble length & 839 elements \\
preamble elements & $N_{b}=16$ \\
preamble format & 0 and 2 \\
RACH configuration index & $c_{f}=1$ to 5 \\
preamble element rate & $\mathrm{R}=1.048 \mathrm{M}$ elements \\
overload threshold & 0.0752 and 0.038 \\
VM2M data rate & $R_{M}=R N_{M} / N_{b}$ \\
one backoff period & $18.51 \mu \mathrm{s}$ \\
Superframe Beacon interval & $540 / c_{f}$ \\
VM2M superframe duration & 41 and 82 backoff period \\
VM2M data packet size & 30 bytes with header \\
\hline
\end{tabular}

\subsubsection{Experiment 1: Combinations of preamble formats (PF) and con- figuration parameters $\left(c_{f}\right)$}

In the first experiment we tried to investigate how $\mathrm{CCH}$ overlay shares the resources with $\mathrm{H} 2 \mathrm{H}$ traffic in $\mathrm{RACH}$. We tried to find the best access parameters for OBUs in $\mathrm{CCH}$, without sacrifice performance of $\mathrm{H} 2 \mathrm{H}$ requests. In this experiments we evaluate the performance of $\mathrm{H} 2 \mathrm{H}$ and VM2M traffic with different combinations of $\mathrm{PF}$ and $c_{f}$ values

\section{Performance evaluation for $\mathrm{H} 2 \mathrm{H}$ traffic}

In numerical evaluation we deploy analytical model from [61] in order to evaluate feasible combinations of formats and configuration parameters for simultaneous VM2M and $\mathrm{H} 2 \mathrm{H}$ access on $\mathrm{RACH}$. We assume that number of codes per cell is $\mathrm{N}=64$, number of preambles reserved for handoff is $N_{h}=10$ and number of preambles reserved for physical 
layer of VM2M overlay is $N_{M}=8$. This leaves $N_{i}=46$ preambles for $\mathrm{H} 2 \mathrm{H}$ access. One data bit in overlay VM2M network is spread over $N_{b}=16$ preamble elements. Required detection threshold for format 0 is $10 \log E_{p} / N_{0}=18 \mathrm{~dB}$ and for format 2 threshold is $15 \mathrm{~dB}$. The corresponding mean ratio of bit energy and noise spectral power density for format 0 is $10 \log E_{b} / N_{0}=-11.23 \mathrm{~dB}$ and for format 2 is $-14.2 \mathrm{~dB}$. The corresponding overload thresholds are $\mathrm{T} 1=0.0752$ and 0.038 .

For third handshake step in which L2/L3 messages are transmitted by fewer terminals, we assume bandwidth to data rate ratio of $1(\mathrm{~W} 3 / \mathrm{R} 3=1)$ and the mean ratio of bit energy and noise spectral power density is $10 \log E_{b} / N=-5 d B$ [74]. We consider maximum number of colliding terminals to be 5 .

For both $\mathrm{H} 2 \mathrm{H}$ and M2M overload cases, we modeled inter-cell interference as a Gaussian random variable with mean $k_{m, 1}=k_{m, 3}=0.247$ and standard deviation $k_{v, 1}=k_{v, 3}=$ 0.078. White noise density was set at $n_{0}=4 \cdot 10^{-21} \mathrm{~W} / \mathrm{Hz}$.

Our first set of experiments evaluates the capacity of $\mathrm{H} 2 \mathrm{H}$ layer when $N_{M}=8$ preambles are permanently active as physical layer of VM2M overlay and intensity of $\mathrm{H} 2 \mathrm{H}$ requests is variable. Second set of experiments evaluates the capacity of VM2M overlay under varying intensity of $\mathrm{H} 2 \mathrm{H}$ requests. In both experiment groups we use same preamble formats (denoted as PF for short) and RACH configuration parameters. By comparing results from two experiment groups we discuss mutual impact of VM2M and $\mathrm{H} 2 \mathrm{H}$ traffic types. 


\section{H2H performance for formats 0 and 2 with minimal configuration parameters}

In first experiment we compare scenarios of $c_{f}=2, \mathrm{PF}=0$ with $c_{f}=1, \mathrm{PF}=2$ when $\mathrm{H} 2 \mathrm{H}$ request arrival rate is varying between 20 and 220 requests per second. These $\mathrm{RACH}$ configurations are depicted in fig. 3.2. Although RACH bandwidth allocations look similar performance metrics show some differences between them. From the figure we could see that the combination $\mathrm{PF}=0$ with $c_{f}=2$ means that 2 independent PRACH resources are available in one LTE frame. Each PRACH resource has preamble duration of $800 \mu$ s. Preamble format 2 with $c_{f}=1$ means that there is a single PRACH resource in the LTE frame which consists of two consecutive subframes. However, the same preamble is transmitted twice with total duration of $1600 \mu$ s. Therefore second combination will gain in power budget but lose one time opportunity per LTE frame. The improvement in power budget is

$$
\begin{aligned}
10 \log _{10}\left(2 E_{s} / N_{0}\right) & =10 \log _{10}(2)+10 \log _{10}\left(E_{p} / N_{0}\right) \\
& =3 d B+10 \log _{10}\left(E_{p} / N_{0}\right)
\end{aligned}
$$

where, $E_{s} / N_{0}$ is energy per symbol to noise power spectral density and $E_{p} / N_{0}$ represents preamble sequence energy to noise power density per $\mathrm{Hz}$. This means that preamble detection threshold for format 2 is $3 \mathrm{~dB}$ lower than threshold for format 0 , i.e. they are equal to $15 \mathrm{~dB}$ and $18 \mathrm{~dB}$ respectively. 


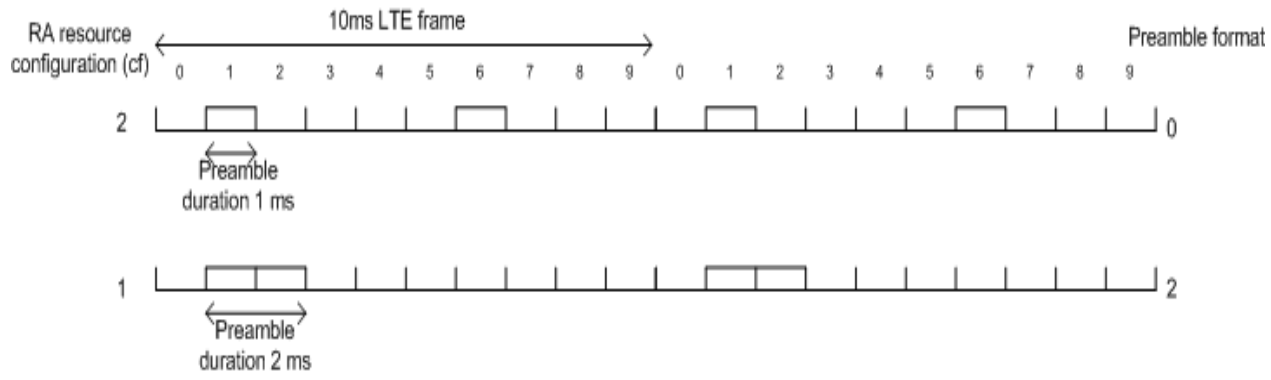

Figure 3.2: Random access resource allocation for $\mathrm{PF}=0$ with $c_{f}=2$ and $\mathrm{PF}=2$ with $c_{f}=1$

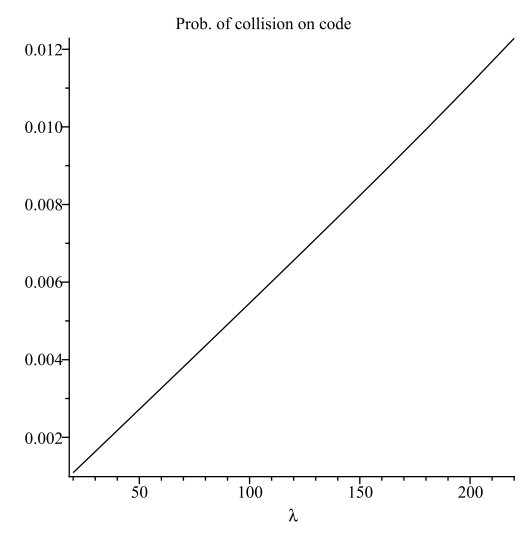

(a) Probability of code collision on PRACH

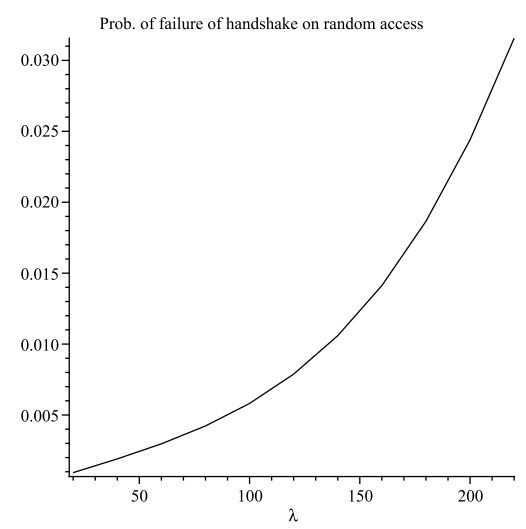

(c) Probability of failure to access on PRACH

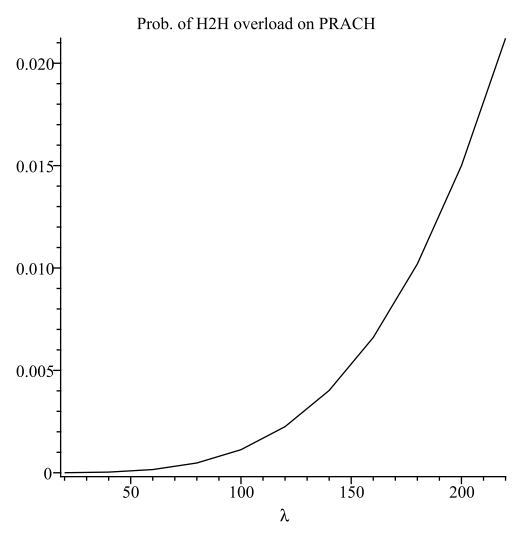

(b) Probability of PRACH overload

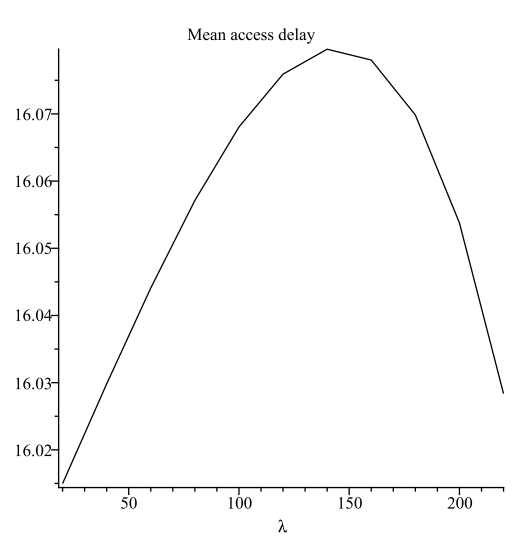

(d) H2H Mean Access Delay in ms

Figure 3.3: Performance of $\mathrm{H} 2 \mathrm{H}$ handshake for $P F=0 c_{f}=2$ 

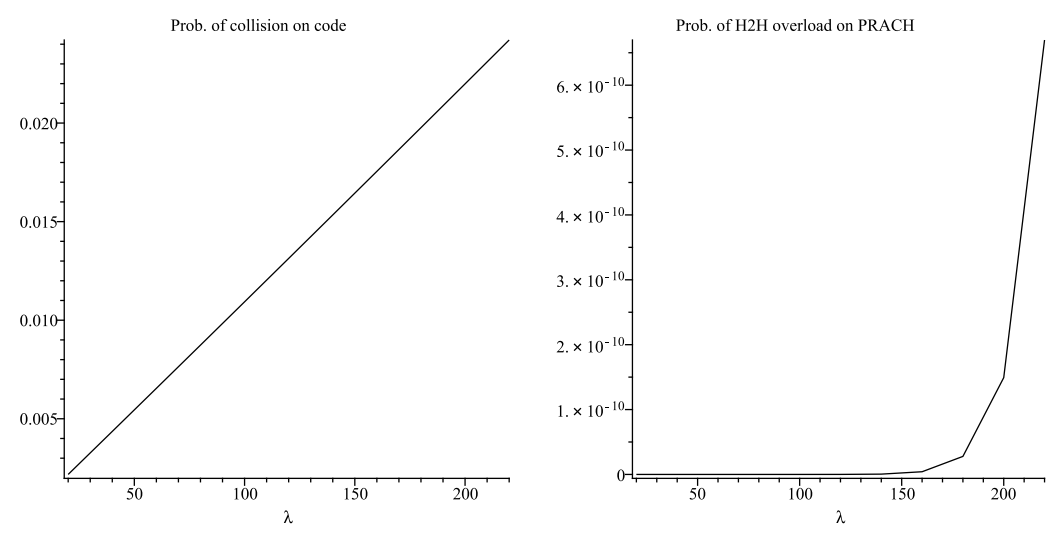

(a) Probability of code collision on

(b) Probability of PRACH overload PRACH

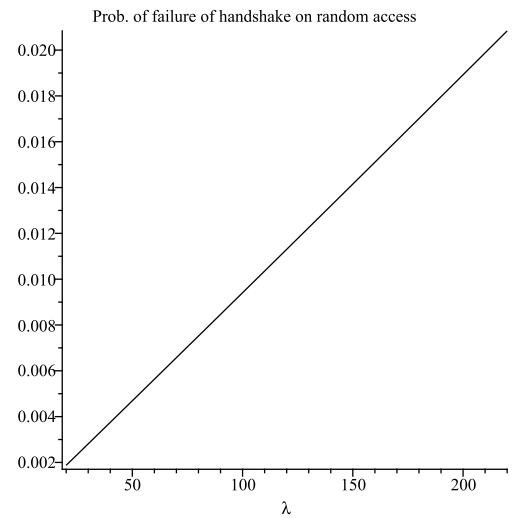

(c) Probability of failure to access on PRACH

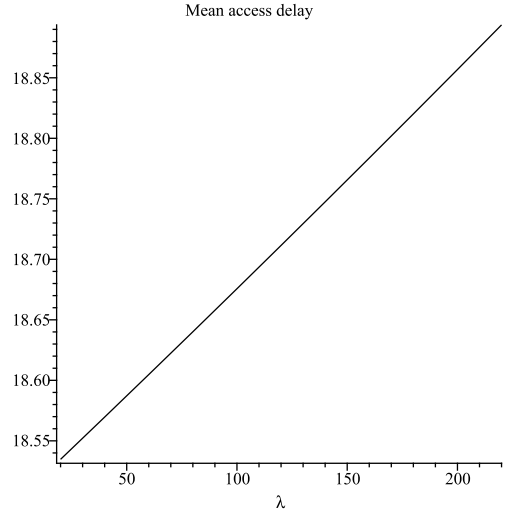

(d) Mean Access Delay in ms

Figure 3.4: Performance of $\mathrm{H} 2 \mathrm{H}$ handshake for $P F=2$ with $c_{f}=1$

By comparing Figs. 3.3 and 3.4 we observe that preamble collision probability of format 2 (fig. 3.4a) is higher than collision probability for format 0 (fig. 3.3a). This is because of the fact that same preambles that are colliding in the first slot of the frame are repeated in the second subframe for format 2 . The collision probability is increasing linearly because they have not reached the saturation yet. We have $\mathrm{H} 2 \mathrm{H}$ and VM2M traffic and both networks are transmitting in parallel. The offered load of the network has the 
impact on the increase of the rate linearly. By comparing Figs. $3.3 \mathrm{~b}$ and $3.4 \mathrm{~b}$ we conclude that format 2 has almost no SINR violation compared to almost $2 \%$ of overload probability for format 0 under maximal load. Since overload has much more impact on preamble success than just preamble collision, handshake failure probability for format 2 is lower compared to format 0 (Figs. 3.3c and 3.4c). If we impose limit of $2 \%$ of acceptable failure probability then this coincides with $\mathrm{H} 2 \mathrm{H}$ request arrival rate of 180 per second for format 0 and 210 per second for format 2. These values represent $\mathrm{H} 2 \mathrm{H}$ subnetwork capacity in RACH under $2 \%$ limit on failure rate. However, despite higher handshake failure probability, for format 0 mean access delay is around $10 \%$ lower due to double frequency of appearance of RACH resource in LTE frame. This is the only penalty of choice of format 2 over format 0 for $\mathrm{H} 2 \mathrm{H}$ traffic. Mean access delay is calculated for maximally 5 access attempts and this explains shape of delay curve shown in Fig. 3.3d.

\section{H2H performance for formats 0 and 2 with large configuration param-}

\section{eters}

In the second experiment we compare the performance of scenario with preamble format $P F=0, c_{f}=3$ and 5, with preamble format $P F=2$ and $c_{f}=2$. These combinations of parameters provide similar but not the same subframe allocation for RACH and allow roughly fair performance comparison. From the Fig. 3.5 we could see that the preamble format 0 with $c_{f}=3$ has $3 \mathrm{PRACH}$ resources with $0.8 \mathrm{~ms}$ preamble duration in one LTE frame. Similarly combination of format 0 and $c_{f}=5$ has 5 PRACH resources in one LTE frame (with $800 \mu$ s preamble duration in each PRACH resource). Combination of preamble format 2 with $c_{f}=2$ means that preamble duration is $2 \times 800 \mu$ s and in one LTE frame 
$2 \times 2$ subframes are allocated for random access. For this experiment we only show $\mathrm{H} 2 \mathrm{H}$ overload probability, probability of access failure on PRACH and mean access delay.

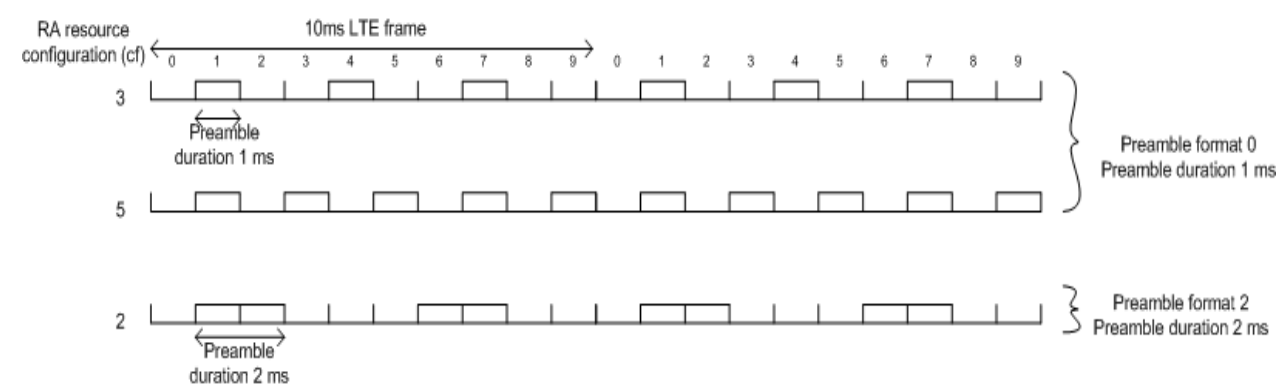

Figure 3.5: Random access resource allocation for $P F=0$ with $c_{f}=3,5$ and $P F=2$ with $c_{f}=2$ 

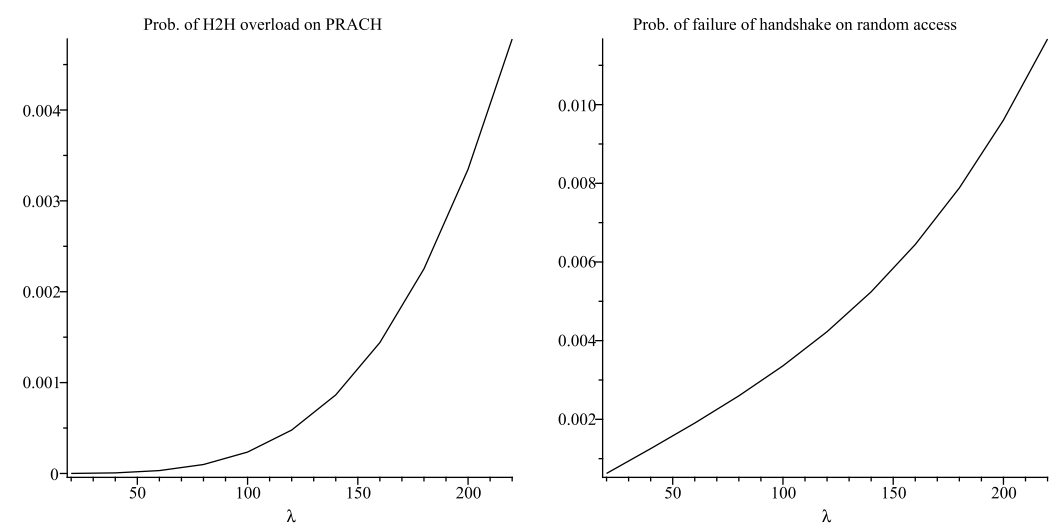

(a) Probability of PRACH overload (b) Probability of failure to access on PRACH

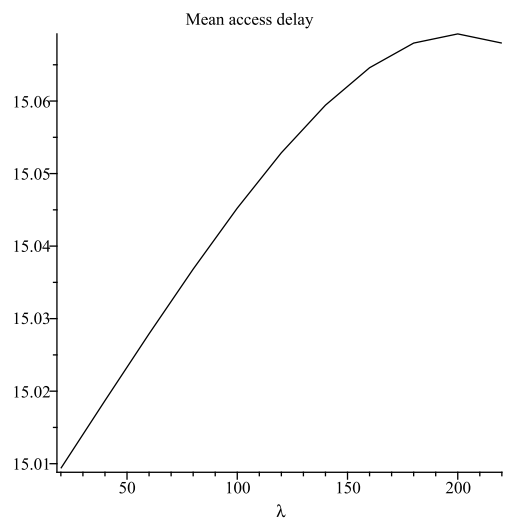

(c) Mean Access Delay

Figure 3.6: Performance of $\mathrm{H} 2 \mathrm{H}$ handshake for $P F=0$ with and $c_{f}=3$ 

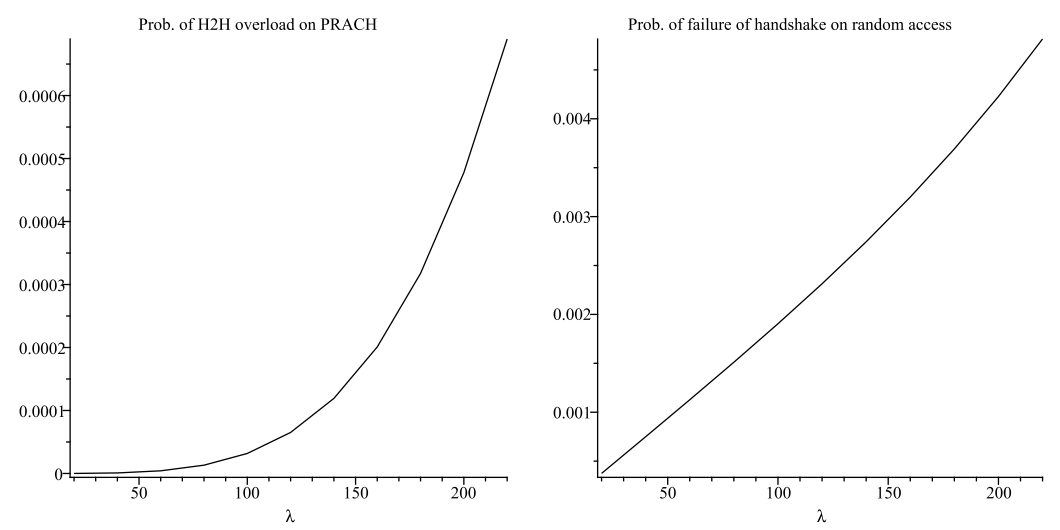

(a) Probability of PRACH overload

(b) Probability of failure to access on PRACH

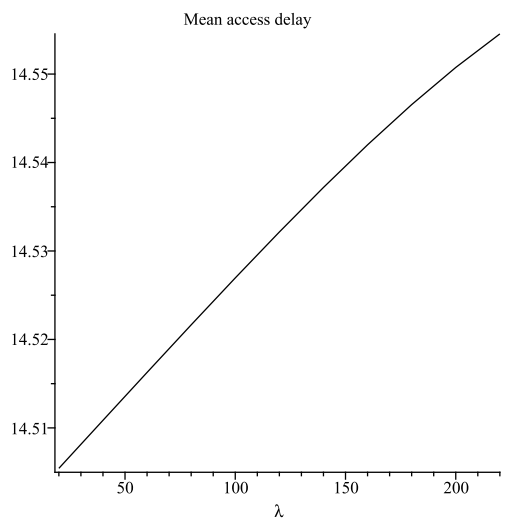

(c) Mean Access Delay

Figure 3.7: Performance of $\mathrm{H} 2 \mathrm{H}$ handshake for $P F=0$ with $c_{f}=5$

Figs. 3.6 and 3.7 show performance of $\mathrm{H} 2 \mathrm{H}$ traffic for preamble format 0 with $\mathrm{RACH}$ configuration index $c_{f}=3$ and 5 respectively when the $\mathrm{H} 2 \mathrm{H}$ request arrival rate varies from 20 to 220 requests per second. For format 0 increased number of PRACH resources in LTE frame improves the performance. The $\mathrm{H} 2 \mathrm{H}$ overload probability of format 0 is getting smaller with increase of $c_{f}$ values. As shown in fig. 3.7a for $c_{f}=5, \mathrm{H} 2 \mathrm{H}$ overload probability is 7 times smaller than for $c_{f}=3$ shown in fig. 3.6a. Failure probability with $c_{f}=5$ as shown in fig. $3.7 \mathrm{~b}$ is smaller than in previous scenario (fig. 3.6b). By comparing 
with fig. 3.6b it can be seen that proportionality coefficient for failure probability is close to $c_{f}$.

Preamble format 2 with double preamble transmission has much lower $\mathrm{H} 2 \mathrm{H}$ overload probability as shown in figure $3.8 \mathrm{a}$ than previous two scenarios involving format 0 (figs. 3.6a and 3.7a). This is contributed to larger power budget for preamble detection. However, the probability of handshake failure as shown in figure $3.8 \mathrm{~b}$ is about the same for scenario when $P F=0$ and $c_{f}=3$ and slightly larger than scenario when $P F=5$ and $c_{f}=5$. Using some extrapolation on graphs $3.6 \mathrm{~b}, 3.7 \mathrm{~b}$ and $3.8 \mathrm{~b}$, we conclude that $\mathrm{H} 2 \mathrm{H}$ subnetwork capacity under $2 \%$ handshake failure rate is around 400 requests per second for format 2 (and $c_{f}=2$ ) and for format 0 it is around 300 for $c_{f}=3$ and around 500 for $c_{f}=5$. On the other hand, total penalty for mean access delay for $\mathrm{H} 2 \mathrm{H}$ traffic is at most $10 \%$ if format 2 is used as can be seen by comparing figs. $3.6 \mathrm{c}, 3.7 \mathrm{c}$ and $3.8 \mathrm{c}$. 

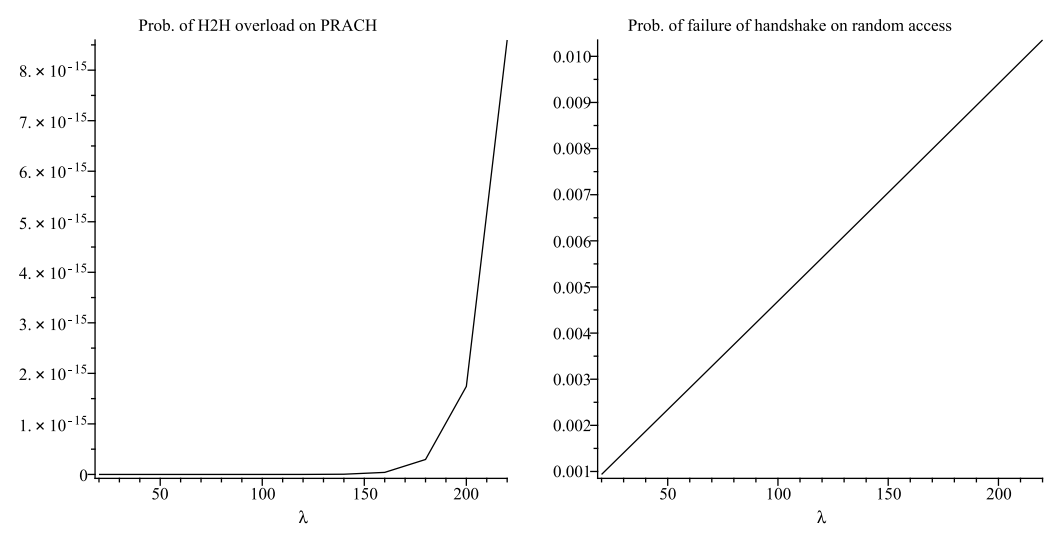

(a) Probability of PRACH overload

(b) Probability of failure to access on PRACH

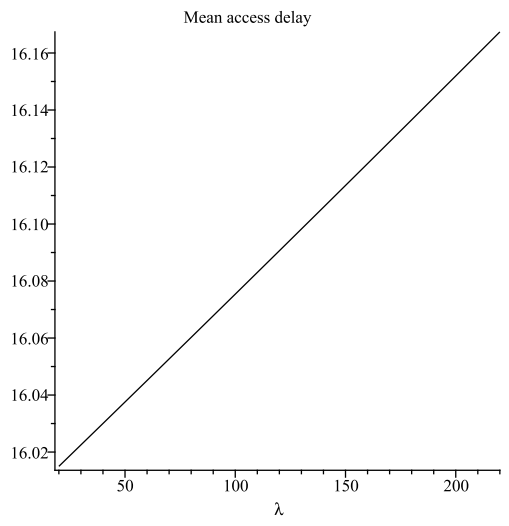

(c) Mean Access Delay

Figure 3.8: Performance of $\mathrm{H} 2 \mathrm{H}$ handshake for $P F=2$ with and $c_{f}=2$

Based on performance data for $\mathrm{H} 2 \mathrm{H}$ traffic it can be seen that for small $\mathrm{RACH}$ allocations format 2 and $c_{f}=1$ offers higher capacity and bit larger access delay compared to use of format 0 and $c_{f}=2$. For large RACH allocations combinations of $P F=2$, $c_{f}=2$ and $P F=0, c_{f}=3$ have similar capacity and access delays, while combination $P F=0, c_{f}=5$ has highest capacity and smallest access delay. Given that their cost in number of RACH subframes is 4, 3 and 5 respectively, we conclude format 0 with increase in PRACH resources is preferable to use by $\mathrm{H} 2 \mathrm{H}$ traffic (given that guard time and cyclic 
prefix limitations are satisfied).

\section{Performance evaluation of VM2M overlay network}

Target of this set of experiments is to evaluate impact of interference from $\mathrm{H} 2 \mathrm{H}$ traffic on VM2M overlay. This interference is demonstrated as Bit Error Rate (BER) in VM2M overlay.

In numerical evaluation we assumed that $N_{M}=8$ preamble codes are dedicated for implementation physical layer of VM2M. Preamble formats are set to $P F=0$ and $P F=2$ respectively. The superframe consists of active and inactive periods according to distances between PRACH resources. This is different from $B O$ and $S O$ parameters which regulate active superframe size and distance between superframes [49]. One backoff period has 20 sequence elements and since one bit requires $N_{b}=16$ preamble elements as a chipping sequence number of bytes that fits into one backoff period is equal to $20 N_{M} /\left(8 N_{b}\right)$. The superframe duration for format 0 is $N_{Z C} / 20=41$ backoff periods and for format 2 it is equal to 82 backoff periods. Consequently format 2 superframe has 102 bytes and for format 0 superframe has 51 bytes. Duration of whole PRACH resource is 54 and 108 backoff periods respectively. The beacon interval between two PRACH resources is $540 / c_{f}$

for both formats. In this work we assume that data packet size is 30 bytes including MAC headers. Data packet should contain cell and node IDs. Among 30 bytes packet size, 10 bytes are reserved for MAC header and other 20 bytes are left for actual MAC data.

MAC parameters for VM2M overlay are set as follows. The backoff exponent $B E$ is initially set to minimum macMinBE $=3$ giving initial backoff window between in the range (0..7). After access failure backoff exponent is incremented by one until it reaches maxi- 
mum value of aMaxBE $=5$. Contention window value is set as $w=2^{B E}$ and maximum number of backoff attempts is set to MaxCSMABackoffs $=5$. Buffer size in VM2M device was set to 3 packets which is sufficient for real time safety messages.

For vehicular traffic we have calculated success probability as the product of probabilities that packet is not corrupted and that packet has not collided with other packet. To investigate the capacity limit of the network we varied number of VM2M devices between 200 and 950. Packet arrival rate per device was varied from 0.2 to 1 arrival per minute. We have conducted experiments to evaluate the fairness in capacity allocation to VM2M subnetwork under constant arrival rate of $\mathrm{H} 2 \mathrm{H}$ traffic. These experiments are VM2M counterparts of experiments for evaluation $\mathrm{H} 2 \mathrm{H}$ performance described in subsections 3.3.1 and 3.3.1 with settings stated in figures 3.2 and 3.5. Our target is to investigate impact of $\mathrm{RACH}$ configurations and formats on performance of VM2M overlay in urban, suburban, rural areas and highways.

\section{VM2M performance for formats 0 and 2 with minimal configuration parameters}

In first experiment we compare scenarios of $c_{f}=2, P F=0$ with $c_{f}=1, P F=$ 2. From previous experiment depicted in section 3.3 .1 when $\mathrm{H} 2 \mathrm{H}$ request arrival rate was varied between 20 and 220 requests per second we have derived overload probability for VM2M traffic. This overload probability represents level of interference coming from $\mathrm{H} 2 \mathrm{H}$ traffic which affects physical layer of VM2M $(\mathrm{CCH})$ overlay. Consequently we have approximated bit error rate (BER) for VM2M data as half of overload probability and packet error rate at the physical layer as $P E R=1-(1-B E R)^{N_{p}}$ where $N_{p}=240$ is the number of bits in the packet. In experiments for VM2M performance $\mathrm{H} 2 \mathrm{H}$ arrival rate was 
kept as constant parameter of 100, 180 and 220 arrivals per second respectively.

First observation is that overload probability and packet error rates are much lower for $\mathrm{RACH}$ format 0 than for format 2 with same time occupancy in LTE frame as shown in figs. 3.9a, 3.9b, 3.10a, 3.10b. This is opposite to our findings for $\mathrm{H} 2 \mathrm{H}$ overload probability which is much higher for RACH format 0 compared to format 2. This means that $\mathrm{H} 2 \mathrm{H}$ twice repeated preambles in format 2 and $c_{f}=1$ are creating much higher interference to VM2M overay than half of traffic intensity with single preamble transmission for format 0 and $c_{f}=2$. High packet error rate will cause packet retransmissions in the overlay which will further cause more packet collisions in 802.15.4 based CSMA/CA scheme which can be seen by comparing figs. $3.9 \mathrm{~h}$ and $3.10 \mathrm{~h}$.

In the next step we evaluate the performance of probability of M2M successful access on PRACH when $\mathrm{H} 2 \mathrm{H}$ arrival rate is 100,180 and 220 per second respectively. For format 0 with $c_{f}=2$, figure $3.9 \mathrm{e}$ shows that in VM2M overlay probability of successful packet transmission is higher than $98.8 \%$ for $220 \mathrm{H} 2 \mathrm{H}$ requests per second and whole range of VM2M node numbers and their traffic intensity. If $2 \%$ of packet failure rate is considered as limit for overlay capacity then up to $220 \mathrm{H} 2 \mathrm{H}$ calls per second ensure smooth operation of VM2M overlay with even 1200 devices. On the other hand, for format 2 there is around 3 to $4 \%$ of failure rate when $\mathrm{H} 2 \mathrm{H}$ request rate is 180 per second as shown in fig. $3.10 \mathrm{~d}$. Beyond that $\mathrm{H} 2 \mathrm{H}$ request intensity VM2M layer can not operate reliably since failure rate reaches $10 \%$ as shown in fig. $3.10 \mathrm{e}$ whereas $\mathrm{H} 2 \mathrm{H}$ counterpart handshake failure probability is only $2 \%$.

Packet transmission time presents period of MAC processing of the packet which at the head of packet queue and access delay is the sum of queuing time and transmission time. 
Both values are presented in milliseconds in figs. $3.9 \mathrm{~g}$ and $3.9 \mathrm{f}$ for $\mathrm{H} 2 \mathrm{H}$ request intensity of 220 per second.

For format 0 and $c_{f}=2$ access delays are approximately half of values obtained when $P F=2$ and $c_{f}=1$ due to smaller distance between the superframes as shown in figs. $3.9 \mathrm{f}$ and 3.10f. It should also be noted that for format $0 \mathrm{M} 2 \mathrm{M}$ access delay is half of $\mathrm{H} 2 \mathrm{H}$ access delay as shown fig. 3.3d. For RACH format $2 \mathrm{M} 2 \mathrm{M}$ access delay is $30 \%$ compared to $\mathrm{H} 2 \mathrm{H}$ access delay. 

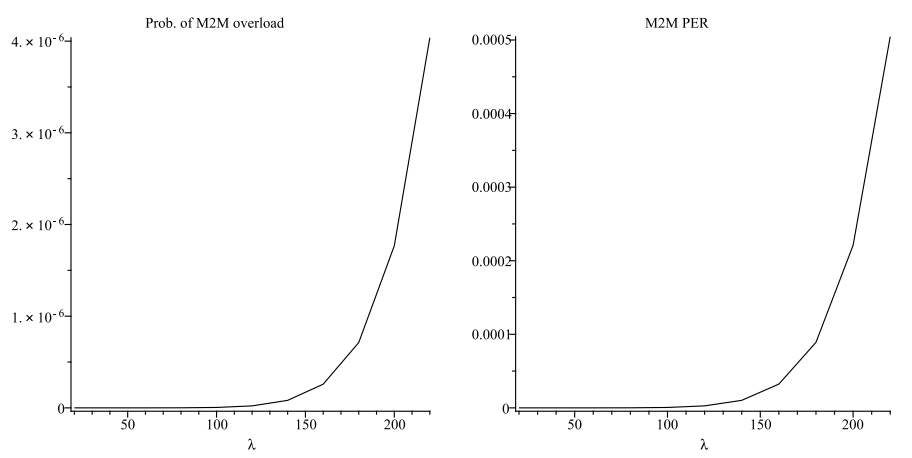

(a) Probability of M2M overload(b) M2M Packet Error Rate on $\mathrm{PRACH}$ caused by $\mathrm{H} 2 \mathrm{H}$ traffic(PER)
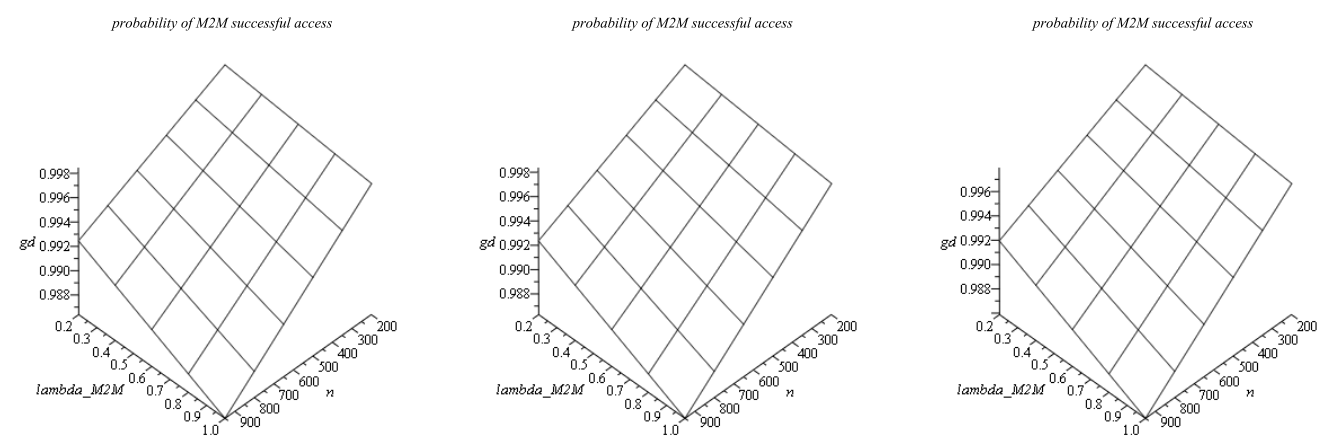

(c) Probability of M2M success-(d) Probability of M2M success-(e) Probability of M2M successful access on PRACH when H2Hful access on PRACH when H2Hful access on PRACH when $\mathrm{H} 2 \mathrm{H}$ arrival rate is $100 \mathrm{calls} / \mathrm{sec}$ arrival rate is $180 \mathrm{calls} / \mathrm{sec}$ arrival rate is 220 calls/sec
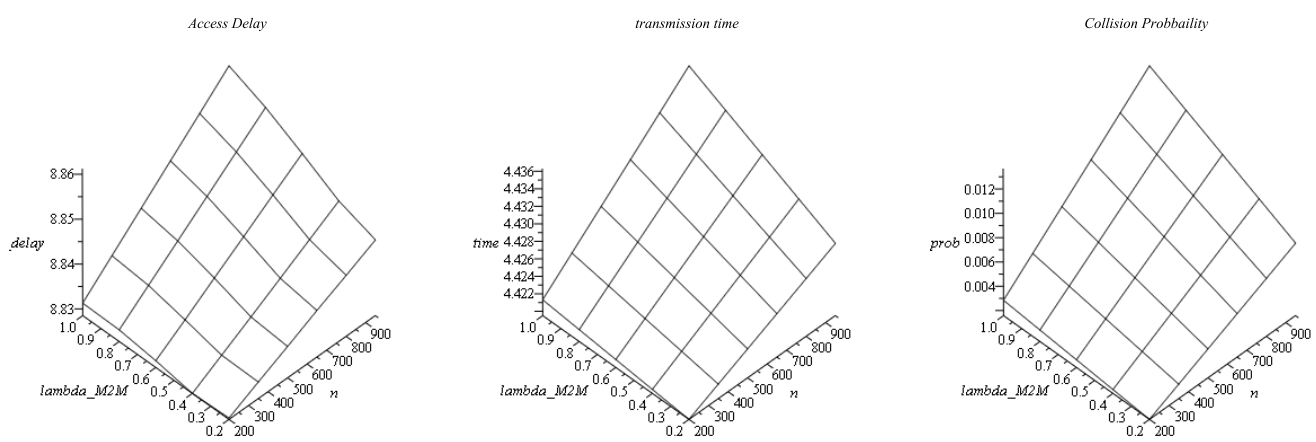

(f) M2M Access Delay when(g) M2M Transmission time(h) M2M Collision Probability $\mathrm{H} 2 \mathrm{H}$ arrival rate is 220 calls perwhen $\mathrm{H} 2 \mathrm{H}$ arrival rate is 220 when $\mathrm{H} 2 \mathrm{H}$ arrival rate is 220 second calls per second calls per second

Figure 3.9: Performance parameters for M2M overlay network for $P F=0$ and $c_{f}=2$ 

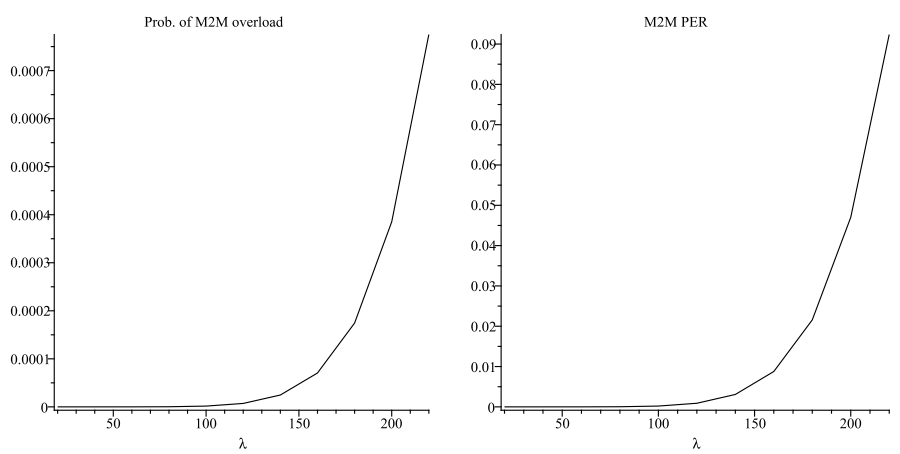

(a) Probability of M2M overload(b) M2M Packet Error Rate on PRACH caused by $\mathrm{H} 2 \mathrm{H}$ traffic(PER)
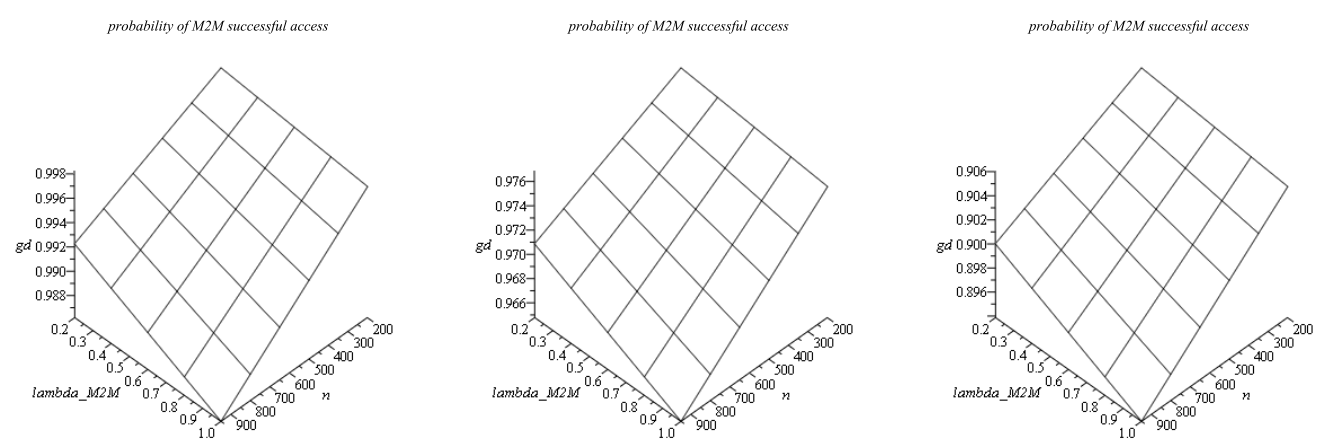

(c) Probability of M2M success-(d) Probability of M2M success-(e) Probability of M2M successful access on PRACH when H2Hful access on PRACH when H2Hful access on PRACH when H2H arrival rate is 100 calls per secondarrival rate is 180 calls per secondarrival rate is 220 calls per second
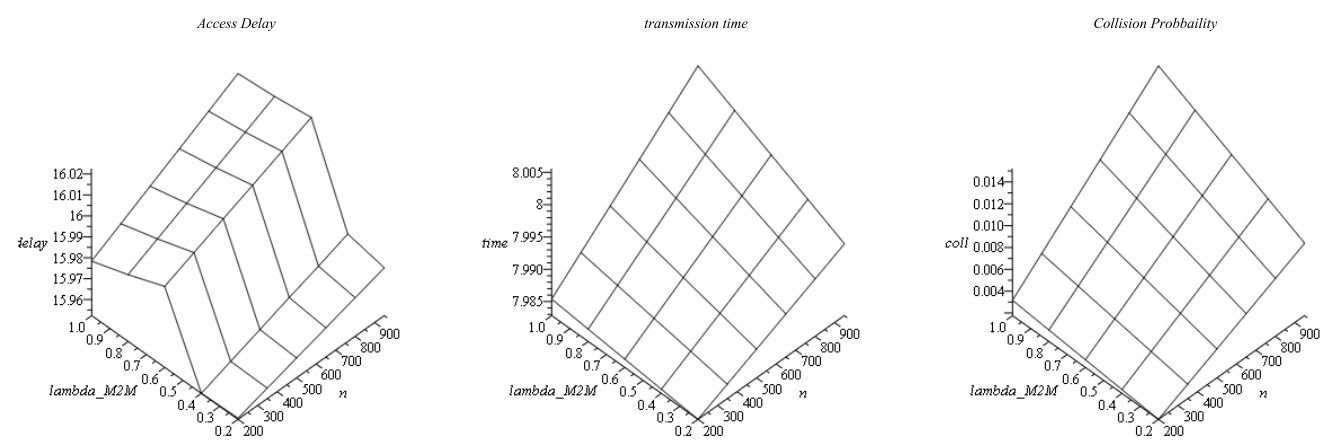

(f) M2M Access Delay when(g) M2M Transmission time(h) M2M Collision Probability $\mathrm{H} 2 \mathrm{H}$ arrival rate is 220 calls perwhen $\mathrm{H} 2 \mathrm{H}$ arrival rate is 220 when $\mathrm{H} 2 \mathrm{H}$ arrival rate is 220 second calls per second calls per second

Figure 3.10: Performance parameters for M2M overlay network for $P F=2$ and $c_{f}=1$ 
Chapter 3: Non priority CSMA/CA overlay network to support vehicular traffic over LTE

\section{VM2M performance for formats 0 and 2 with large configuration parameters}

In the second experiment we consider scenarios as shown in figure 3.5 . We evaluate the performance of following scenarios with close subframe allocations:

(a) $P F=0, c_{f}=3$ and 5

(b) $P F=2, c_{f}=2$

Figures 3.11 and 3.12 show VM2M performance descriptors calculated for preamble format 0 when $\mathrm{H} 2 \mathrm{H}$ request arrival rate is set as a parameter. For overload probability and packet error rate $\mathrm{H} 2 \mathrm{H}$ request rate varies 40 to 220 per second and for other descriptors it is set to 200 requests per second. Number of M2M devices varies from 200 to 950 per second and VM2M arrival rate varies between 0.2 and 1 packet arrival per minute.

The M2M overload probability with $c_{f}=5$ is smaller than $c_{f}=3$ because the superframe size is larger. The probabilities of successful access for both scenarios are close to 1 because the PER for both scenarios (fig. 3.11b and 3.12b) is very small. M2M overload probability is getting smaller with the increase of $c_{f}$ for preamble format 0 as shown in figure $3.11 \mathrm{a}$ and 3.12a. However, M2M overload probability for format 2 (fig. 3.13a) is higher than in format 0 due to repeating of preamble transmission in $\mathrm{RACH}$ resource.

The access delay for format 0 decreases with the increase of number of PRACH resources $c_{f}=3$ and 5. Delay for format 2 and $c_{f}=2$ is larger compared to format 0 due to larger distance between the superframes. However, all M2M access delays are lower than equivalent $\mathrm{H} 2 \mathrm{H}$ access delays. 


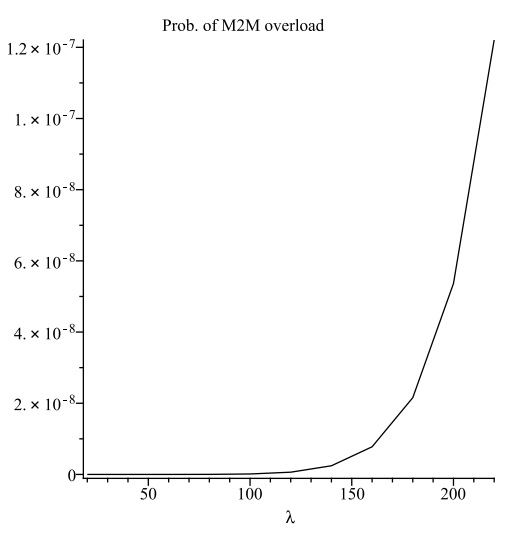

(a) Probability of M2M overload on $\mathrm{PRACH}$ caused by $\mathrm{H} 2 \mathrm{H}$ traffic

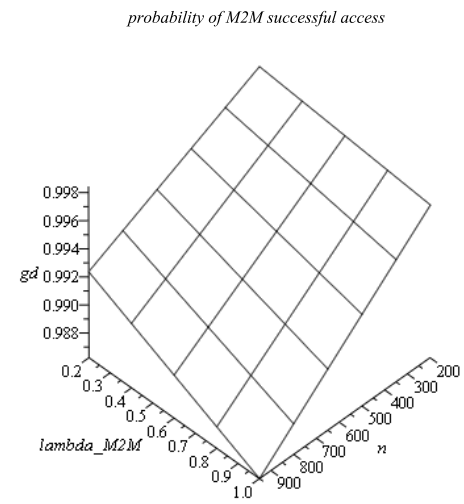

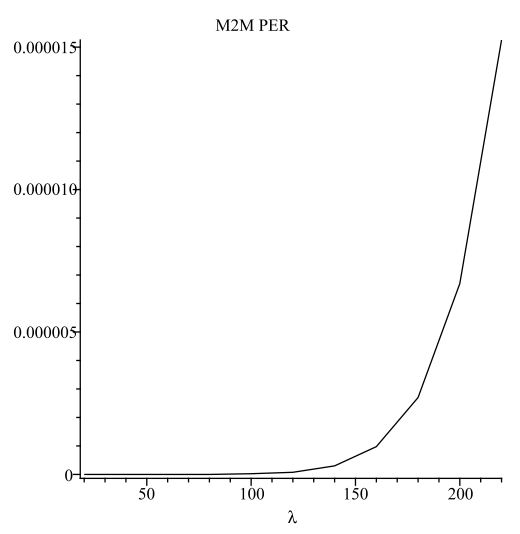

(b) M2M Packet Error Rate (PER)

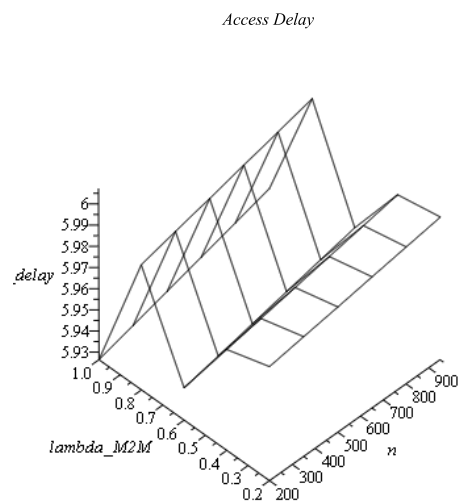

(c) Probability of M2M successful ac-(d) M2M Access Delay when $\mathrm{H} 2 \mathrm{H}$ arcess on $\mathrm{PRACH}$ when $\mathrm{H} 2 \mathrm{H}$ arrival raterival rate is 220 calls per second is 220 calls per second
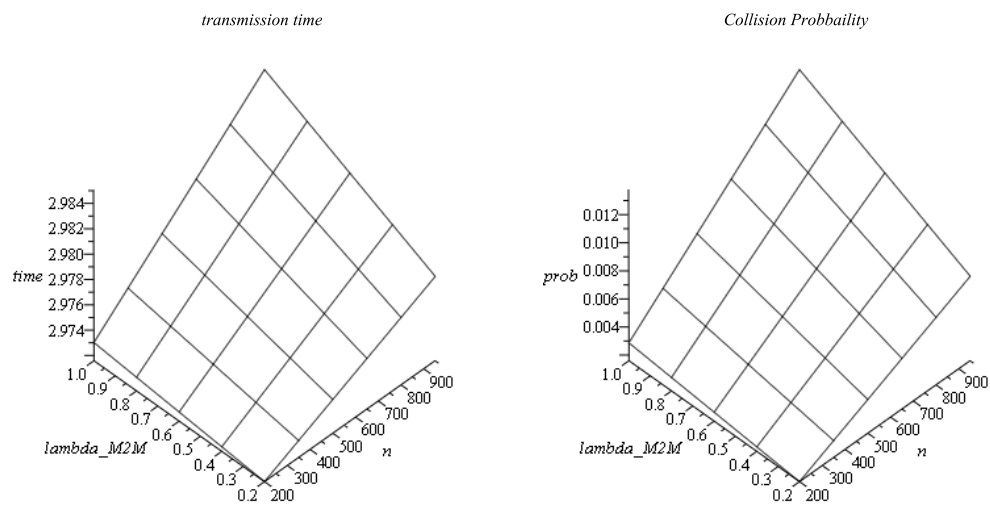

(e) M2M Transmission time when(f) M2M Collision Probability when $\mathrm{H} 2 \mathrm{H}$ arrival rate is 220 calls per sec- $\mathrm{H} 2 \mathrm{H}$ arrival rate is 220 calls per second ond

Figure 3.11: Performance parameters for $42 \mathrm{M}$ overlay network for $P F=0$ and $c_{f}=3$ 


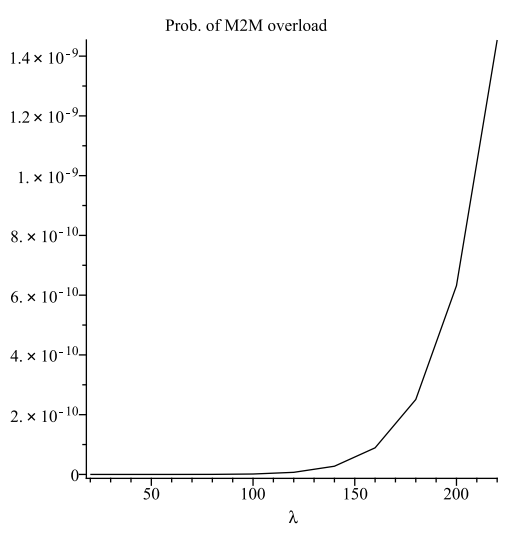

(a) Probability of M2M overload on PRACH caused by $\mathrm{H} 2 \mathrm{H}$ traffic

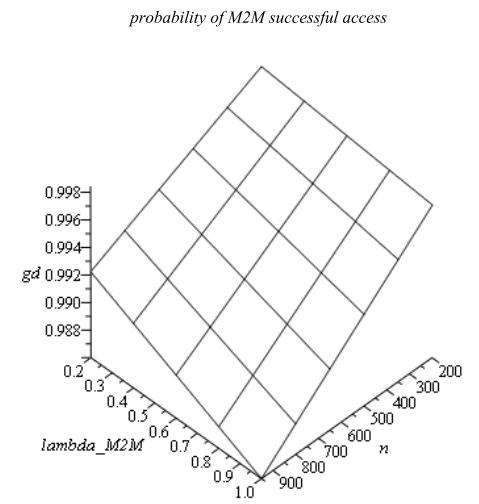

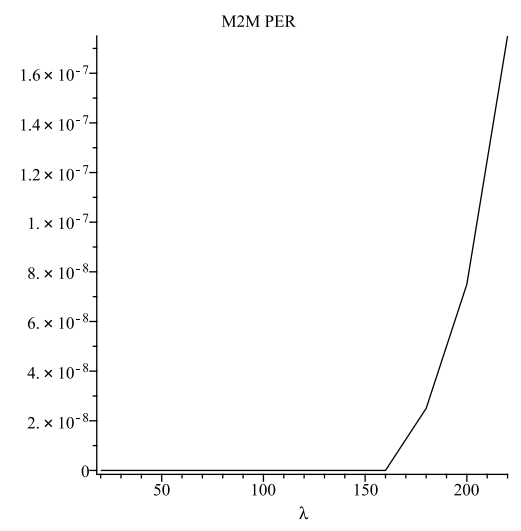

(b) M2M Packet Error Rate (PER)

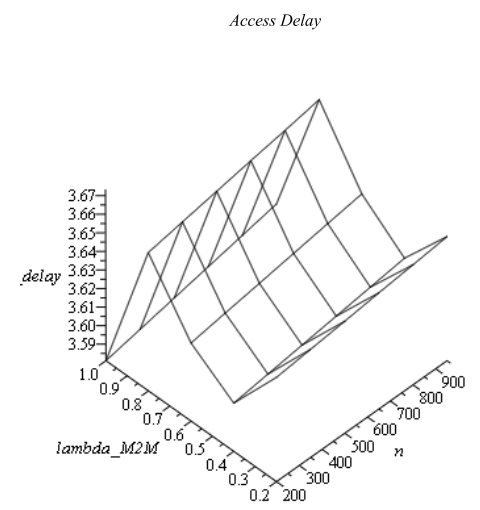

(c) Probability of M2M successful ac-(d) M2M Access Delay when $\mathrm{H} 2 \mathrm{H}$ arcess on $\mathrm{PRACH}$ when $\mathrm{H} 2 \mathrm{H}$ arrival raterival rate is 220 calls per second is 220 calls per second
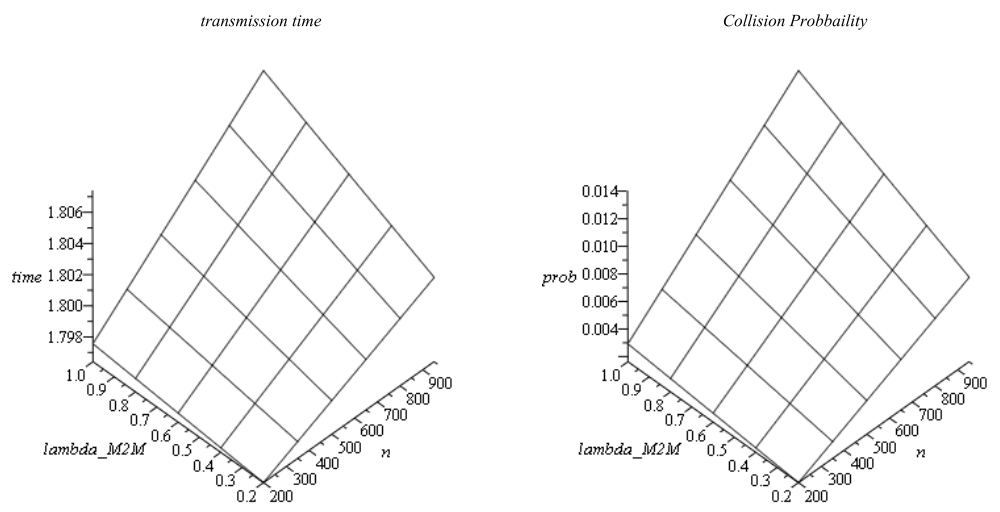

(e) M2M Transmission time when(f) M2M Collision Probability when $\mathrm{H} 2 \mathrm{H}$ arrival rate is 220 calls per sec- $\mathrm{H} 2 \mathrm{H}$ arrival rate is 220 calls per second ond

Figure 3.12: Performance parameters for $\mathrm{M}_{8} \mathrm{M}$ overlay network for $P F=0$ and $c_{f}=5$ 

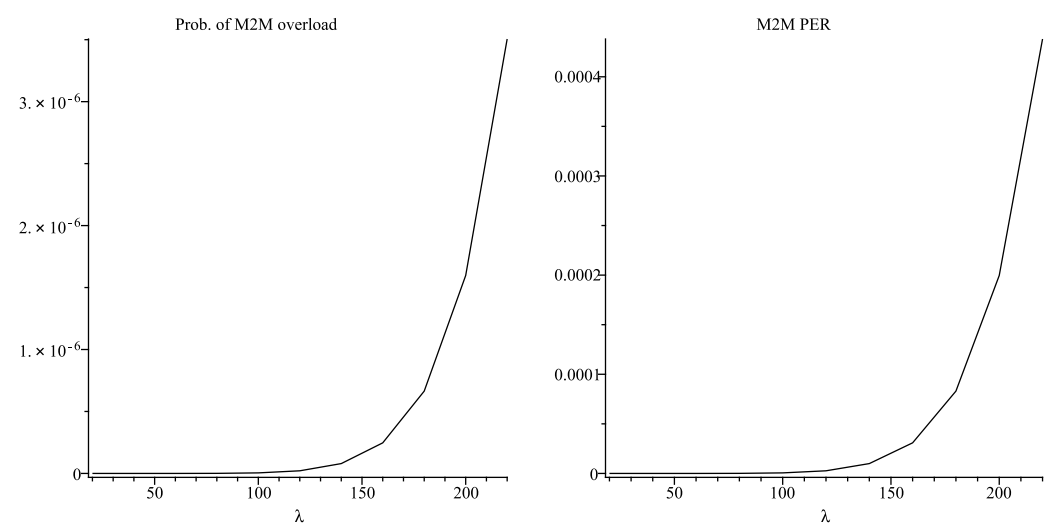

(a) Probability of M2M overload on

(b) M2M Packet Error Rate (PER) PRACH caused by $\mathrm{H} 2 \mathrm{H}$ traffic
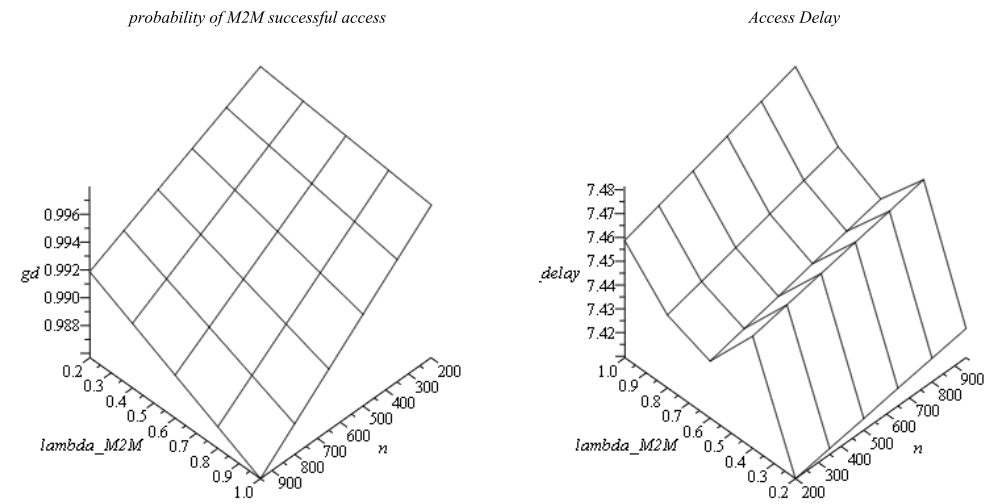

(c) Probability of M2M successful ac-(d) M2M Access Delay when H2H arcess on PRACH when $\mathrm{H} 2 \mathrm{H}$ arrival raterival rate is 220 calls per second is 220 calls per second
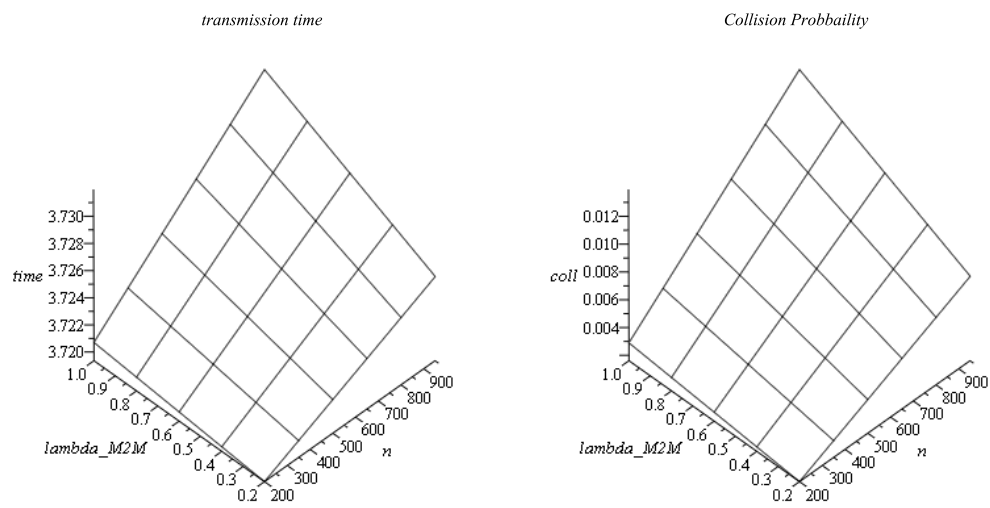

(e) M2M Transmission time when(f) M2M Collision Probability when $\mathrm{H} 2 \mathrm{H}$ arrival rate is 220 calls per sec- $\mathrm{H} 2 \mathrm{H}$ arrival rate is 220 calls per second ond

Figure 3.13: Performance parameters for M2M overlay network for $P F=2$ and $c_{f}=2$ 
Chapter 3: Non priority CSMA/CA overlay network to support vehicular traffic over LTE

\section{Discussion}

Our results have shown that for small $\mathrm{RACH}$ allocations $\mathrm{H} 2 \mathrm{H}$ subnetwork has $20 \%$ larger capacity for format 0 and $c_{f}=2$ compared to format 2 and $c_{f}=1$ (220 requests per second versus 180 requests per second). $\mathrm{CCH}$ overlay under format 0 and $c_{f}=2$ on the other hand can accommodate around 1000 OBUs each transmitting one packet per minute with simultaneous arrival of $220 \mathrm{H} 2 \mathrm{H}$ requests since probability of successful access is higher than 0.98. Unfortunately for format 2 and $c_{f}=1$ the same $\mathrm{CCH}$ capacity can be achieved only under $100 \mathrm{H} 2 \mathrm{H}$ requests per second. This puts $\mathrm{CCH}$ overlay operation in jeopardy if $\mathrm{H} 2 \mathrm{H}$ request rate is not limited.

For larger RACH allocation format 2 with $c_{f}=2$ offers just $20-30 \%$ of increased capacity and 5\% larger delay compared to format 0 and $c_{f}=3$. This barely matches $30 \%$ increased subframe allocation. Configuration $c_{f}=5$ with format 0 has $25 \%$ increase in subframe allocation and similar increase in capacity with shorter access time. With respect to capacity of $\mathrm{CCH}$ overlay all three combinations can accommodate 1000 OBUs with transmission rate of 1 packet per minute. However, by extrapolation we note combinations with format 0 can work smoothly with up to 1500 OBUs while combination with format 2 can accommodate at most 1100-1200 OBUs with transmitting one packet per minute.

We also note that, for all cases access delays for VM2M (CCH) overlay are significantly lower compared to $\mathrm{H} 2 \mathrm{H}$ subnetwork. Regardless of RACH format increasing the frame configuration parameter will almost linearly increase the capacity of $\mathrm{H} 2 \mathrm{H}$ and VM2M layer and will decrease the access time. However with high frequency of RACH allocations care has to be taken to avoid interference with PUSCH transmission in surrounding cells which can increase RACH interference and decrease overlay capacity. 
Unfortunately, the choice of preamble format for $\mathrm{H} 2 \mathrm{H}$ and VM2M depends on environment. For sub-urban, rural and highway scenarios RACH format 2 (or even 3) might be necessary due to large velocity and round trip time. Use of format 2 increases the power budget and gives the priority to $\mathrm{H} 2 \mathrm{H}$ traffic since each preamble is transmitted twice. VM2M overlay network is penalized in this scenario since it receives higher interference. For urban environment RACH format 0 can be used and capacity can be increased with increasing RACH configuration parameter.

The use of overlay network for the $\mathrm{CCH}$ affects $\mathrm{H} 2 \mathrm{H}$ traffic with the deterministic impact of $N_{M}$ active preambles. On the other hand variable rates of $\mathrm{H} 2 \mathrm{H}$ requests present variable (random) interference to VM2M overlay which is a much bigger challenge. Capacity of M2M overlay can be increased by increasing the number of preambles $N_{M}$ used in the VM2M physical layer. This is also beneficial from the aspect of CSMA/CA since superframe capacity will increase and packet access can be spread more evenly over the superframe duration.

\subsubsection{Experiment 2: PRACH bandwidth partitioning for VM2M}

In the second experiment, we studied three deployment scenarios over overlay networks under different situations of low, moderate and high VM2M traffic scenarios. We have investigated the physical layer limit and capacity of both sub-networks by partitioning the PRACH bandwidth for VM2M overlay networks through preamble codes. From the first experiment we understand that we might need preamble format 2 which is suitable for both urban and rural areas with large cells without sacrificing performance of $\mathrm{H} 2 \mathrm{H}$ traffic. In this experiment we consider $c_{f}=1 \mathrm{PRACH}$ resources and preamble formats (PF) 2 respectively. 
From the Fig. 3.14 we could see that the combination $\mathrm{PF}=2$ with $c_{f}=1$ means that there is a single PRACH resource in the LTE frame which consists of two consecutive subframes. Therefore $P F=2$ gains in power budget, but lose one time opportunity per LTE frame. The preamble detection threshold is $15 \mathrm{~dB}$. The corresponding mean ratio of bit energy and noise spectral power density is $10 \log \frac{E_{b}}{N_{0}}=-14.2 \mathrm{~dB}$. The corresponding overload threshold is $\mathrm{T} 2=0.038$. The superframe duration is equal to 82 backoff periods. Consequently format 2 superframe with $N_{M}=4,8$ and 12 has 51, 102 and 153 bytes. Duration of whole PRACH resource is 108 backoff periods. The beacon interval between two PRACH resources is $540 / c_{f}$. In this work, we assume that the data packet size is 30 bytes including MAC headers. Data packet should contain cell and node IDs. Among 30 bytes packet size, 10 bytes are reserved for MAC header and other 20 bytes are left for actual MAC data.

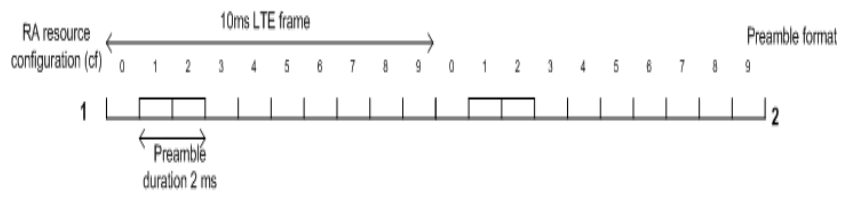

Figure 3.14: Random access resource allocation for $\mathrm{PF}=2$ with $c_{f}=1$

We evaluate the capacity of VM2M overlay under varying intensity of $\mathrm{H} 2 \mathrm{H}$ requests and preamble codes. We varied number of $N_{M}$ codes between 4,8 and 12. The target of this experiment is to evaluate the range of acceptable working limits of VM2M overlay. The interference of $\mathrm{H} 2 \mathrm{H}$ traffic is demonstrated as Bit Error Rate (BER) in VM2M overlay. The superframe consists of active and inactive periods according to distances between PRACH resources. This is different from $B O$ and $S O$ parameters which regulate active superframe size and distance between superframes [49]. We have calculated the success probability as the product of probabilities that packet is not corrupted and that packet has not collided 
with other packet. Packet arrival rate per device was varied from 0.2 to 1 arrival per minute. We have conducted experiments to evaluate the fairness in capacity allocation to VM2M subnetwork under constant arrival rate of $\mathrm{H} 2 \mathrm{H}$ traffic. Our target is to investigate the impact of $N_{M}$, RACH configurations and formats on the performance of VM2M overlay in urban, suburban, rural areas and highways. We have derived overload probability for VM2M traffic when $\mathrm{H} 2 \mathrm{H}$ request arrival rate was varied between 20 and 220 requests per second - This overload probability represents the level of interference coming from $\mathrm{H} 2 \mathrm{H}$ traffic which affects physical layer of VM2M (CCH) overlay. Under different $\mathrm{H} 2 \mathrm{H}$ capacity we have approximated bit error rate (BER) for VM2M data as half of overload probability and packet error rate at the physical layer as $P E R=1-(1-B E R)^{N_{p}}$ where $N_{p}=240$ is the number of bits in the packet.

\section{Performance of VM2M and H2H traffic}

In the numerical evaluation, we deploy analytical model from [61] in order to evaluate the capacity and impact of $\mathrm{H} 2 \mathrm{H}$ layer for different combination of $N_{M}$ preambles which are permanently active as physical layer of VM2M overlay and intensity of $\mathrm{H} 2 \mathrm{H}$ requests is variable. By varying the value of $N_{M}$ we are partitioning the PRACH bandwidth and doing the tradeoff between a number of vehicular calls and $\mathrm{H} 2 \mathrm{H}$ calls where both subnetworks can coexist satisfactorily. We are studying three deployment scenarios of overlay networks for different situations. We tried to find the feasible combination of number of dedicated codes for VM2M overlay networks in different application scenarios with preamble formats (PF) and configuration parameters. We pointed out that, $\mathrm{PF}=2$ is suitable for urban, sub-urban, rural areas and especially highways as it provides large guard times, can handle higher 
mobility, provides larger cell and can handle handover calls greatly. We consider scenarios as shown in figure 3.14 and evaluate the performance of following experiments:

$$
P F=2, c_{f}=1 \text { with variable } N_{M}=4,8 \text { and } 12
$$

\section{Case 1: Very low VM2M traffic scenario}

In scenarios like rural areas with a very low stream of vehicles, we could have $N_{M}=4$ dedicated preamble codes for VM2M overlay network and leave rest of the preambles for $\mathrm{H} 2 \mathrm{H}$ traffic. In every cell we have $\mathrm{H} 2 \mathrm{H}$ and VM2M traffic so we always want to have vehicular overlay which is always sufficient for some traffic. We are taking the minimum $N_{M}$ codes so that it could handle the VM2M traffic. We evaluate the performance of both $\mathrm{H} 2 \mathrm{H}$ and overlay network for format 2 with $N_{M}=4$ to evaluates the impact and capacity of the overlay network.

From Fig. 3.16d, we observe that VM2M probability of success under $\mathrm{H} 2 \mathrm{H}$ arrival rate of 220 calls/sec is $98 \%$ for up to $680 \mathrm{VM} 2 \mathrm{M}$ traffic. Where as $\mathrm{H} 2 \mathrm{H}$ failure probability ( 3.15 c) is less than $2 \%$ for up to $220 \mathrm{H} 2 \mathrm{H}$ calls/sec. with very small probability of overload ( 3.15b). It shows that we can accommodate more than $220 \mathrm{H} 2 \mathrm{H}$ calls with restriction of VM2M traffic up to 680 with maximum 1 packet per min. Beyond that VM2M network goes to saturation with increasing packet error rate and collision probability (Figs. 3.16a and $3.16 \mathrm{c}$ ). If up to $2 \%$ of packet failure rate is considered as the limit for $\mathrm{H} 2 \mathrm{H}$ traffic then up to $250 \mathrm{H} 2 \mathrm{H}$ calls per second ensure smooth operation with VM2M overlay with up to 680 devices. 

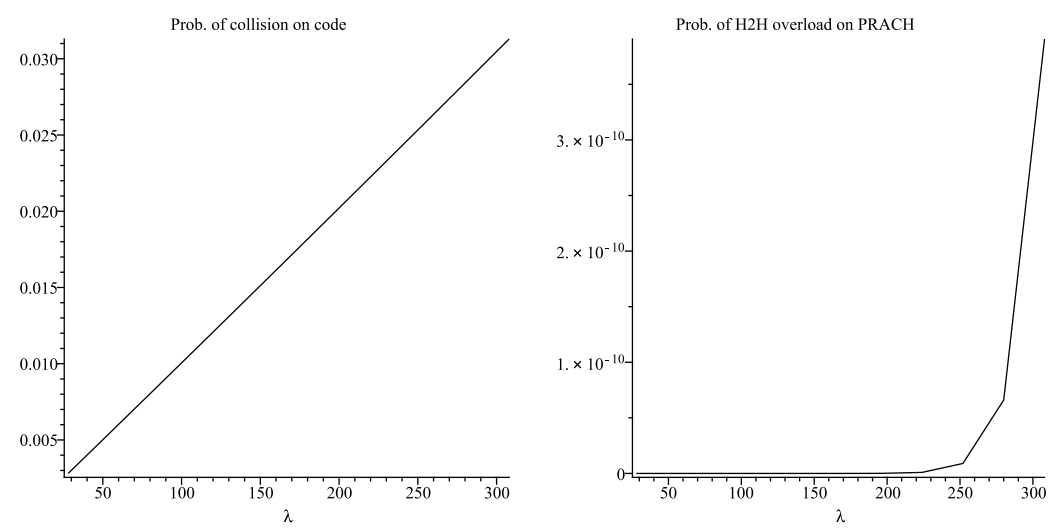

(a) Probability of code collision on

(b) Probability of PRACH overload PRACH

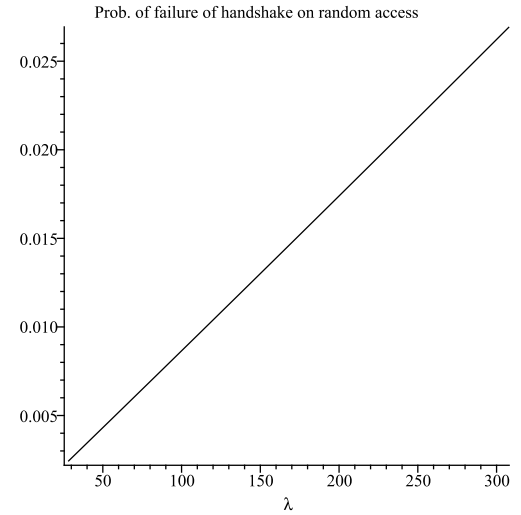

(c) Probability of failure to access on PRACH

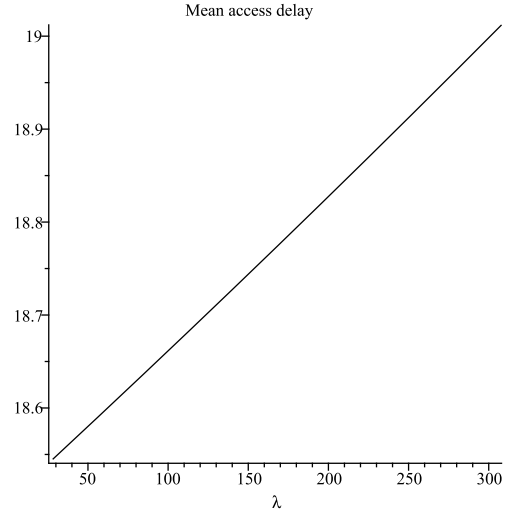

(d) Mean Access Delay

Figure 3.15: Performance of $\mathrm{H} 2 \mathrm{H}$ handshake for $P F=2, N_{M}=4 c_{f}=1$

\section{Case 2: Moderate H2H and VM2M traffic scenario}

In urban, rural, sub-urban areas and highways with moderate traffic situations where we have $\mathrm{H} 2 \mathrm{H}$ and VM2M traffic we could consider $N_{M}=8$. We have 2 networks and we have to accommodate both networks capacity. We tried to find the capacity limit for VM2M traffic, where $\mathrm{CCH}$ overlay network could work properly without sacrificing $\mathrm{H} 2 \mathrm{H}$ traffic. 

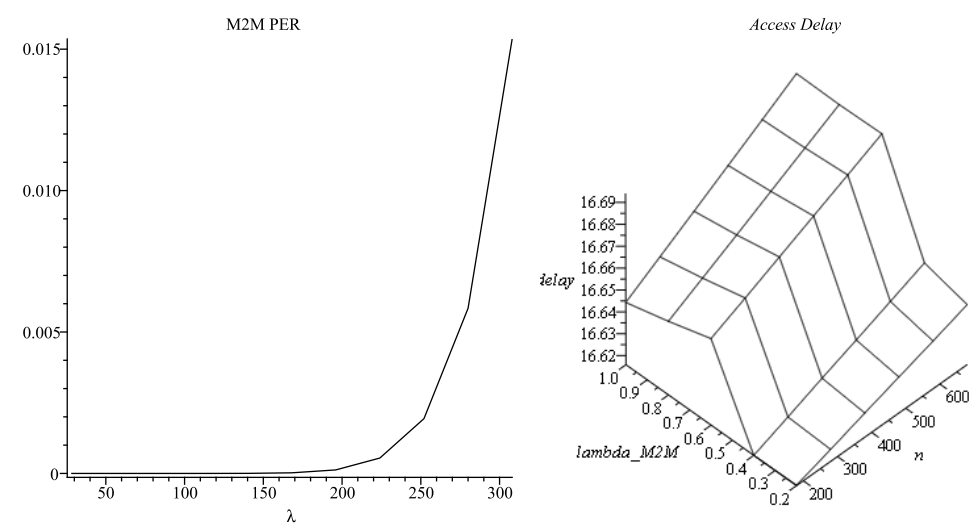

(a) M2M Packet Error Rate (PER)

(b) M2M Access Delay when $\mathrm{H} 2 \mathrm{H}$ arrival rate is 220 calls/sec
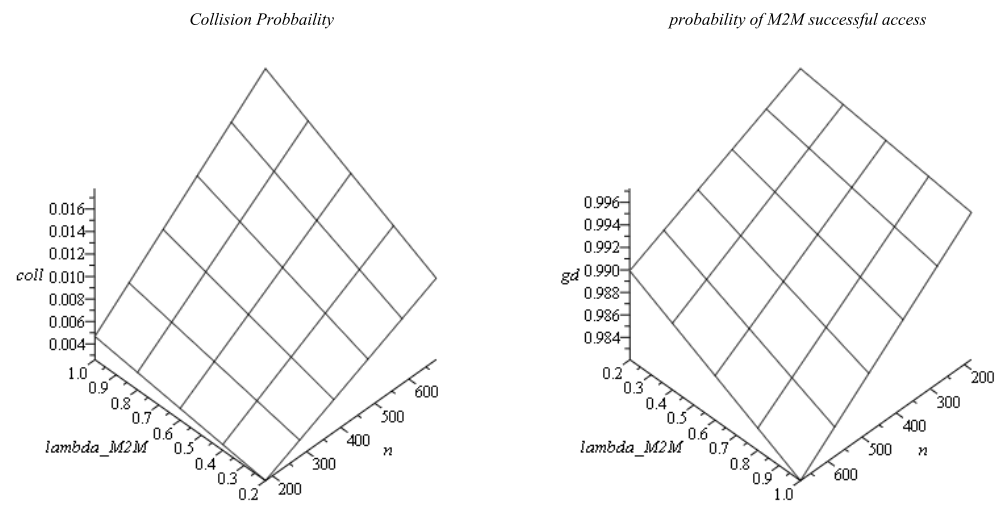

(c) Collision Probability when $\mathrm{H} 2 \mathrm{H}$ ar-(d) Pro. of success when $\mathrm{H} 2 \mathrm{H}$ arrival rival rate is 220 calls/sec rate is 220 calls/sec

Figure 3.16: Performance parameters for VM2M overlay network for $P F=2, N_{M}=4$ and $c_{f}=1$

From Fig. 3.18b we observe that for VM2M traffic scenarios with 900 devices there are around 3 to $4 \%$ of failure rate when $\mathrm{H} 2 \mathrm{H}$ request rate is 180 per second. Beyond that $\mathrm{H} 2 \mathrm{H}$ request intensity VM2M layer can not operate reliably since failure rate reaches $10 \%$ as shown in fig. $3.18 \mathrm{c}$ whereas $\mathrm{H} 2 \mathrm{H}$ counterpart handshake failure probability is only $2 \%$ (Fig. 3.17c). If we impose a limit of $2 \%$ of acceptable failure probability with reasonable $\mathrm{H} 2 \mathrm{H}$ capacity $\left(N_{M}=8\right)$ then this coincides with $\mathrm{H} 2 \mathrm{H}$ request arrival rate of 210 per sec- 
ond. Where as, for VM2M overlay with a limit of $2 \%$ failure probability restricts $\mathrm{H} 2 \mathrm{H}$ request arrival rate of less than 180 calls per second with reasonable VM2M capacity of 900 devices. High packet error rate of VM2M as shown in figure 3.18a has much impact on failure probability. With $N_{M}=8 \mathrm{H} 2 \mathrm{H}$ traffic has almost no SINR violation (Fig. 3.17b) but more than $2 \%$ of collision probability (Fig. 3.17a). VM2M subnetworks access delay is $30 \%$ compared to $\mathrm{H} 2 \mathrm{H}$ access delay.
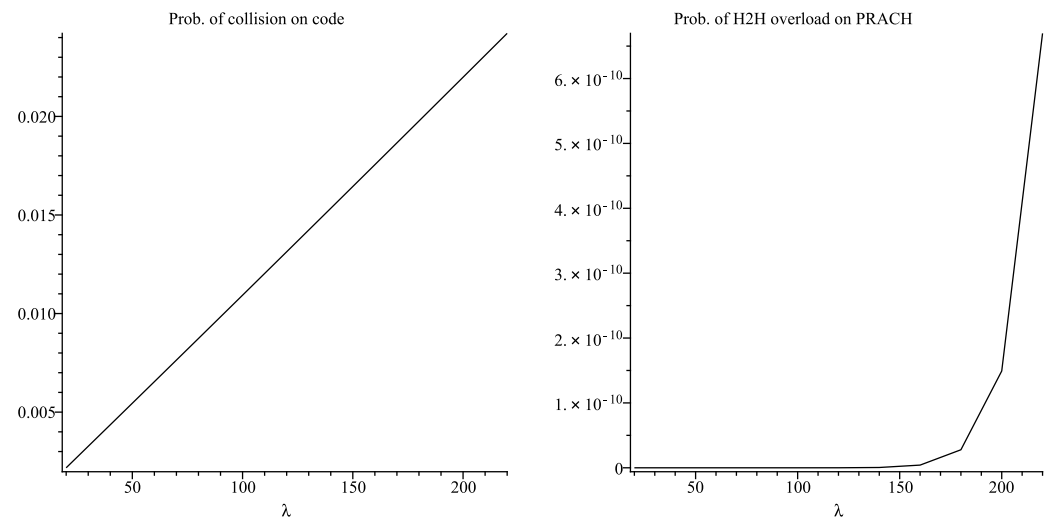

(a) Probability of code collision on

(b) Probability of PRACH overload PRACH

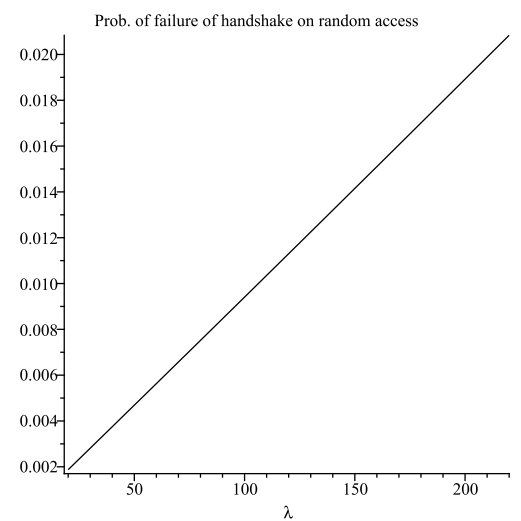

(c) Probability of failure to access on PRACH

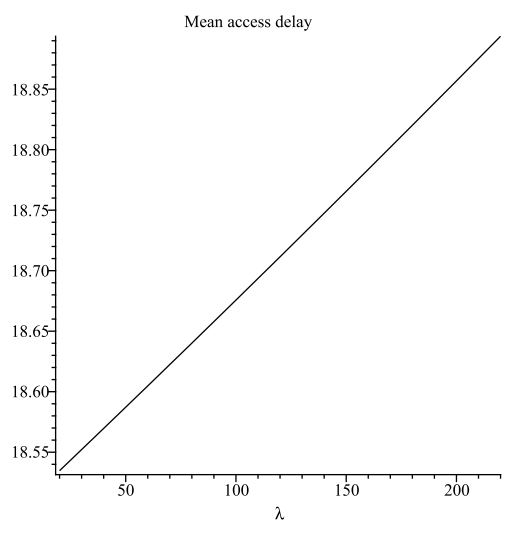

(d) Mean Access Delay in ms

Figure 3.17: Performance of $\mathrm{H} 2 \mathrm{H}$ handshake for $P F=2, N_{M}=8, c_{f}=1$ 

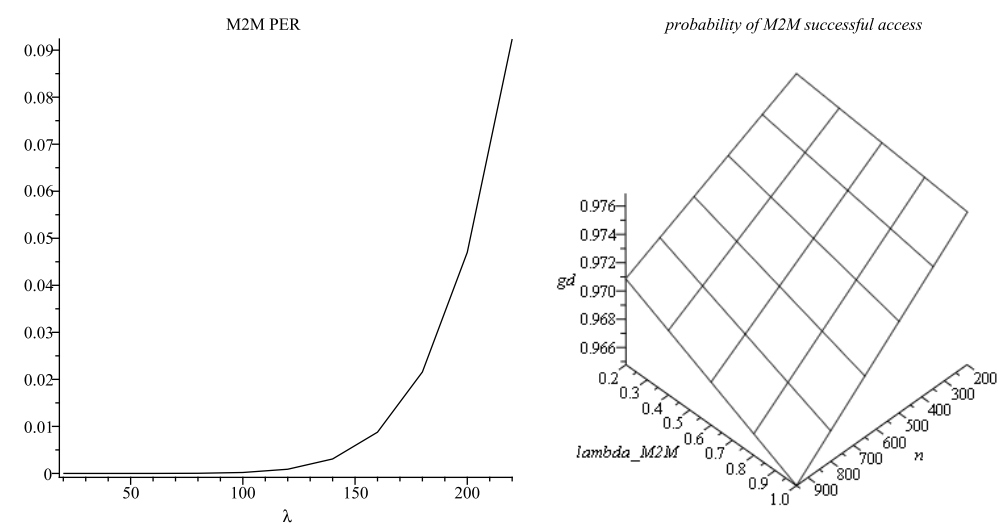

(a) M2M Packet Error Rate (PER)

(b) Pro. of success when $\mathrm{H} 2 \mathrm{H}$ arrival rate is 180 calls/sec
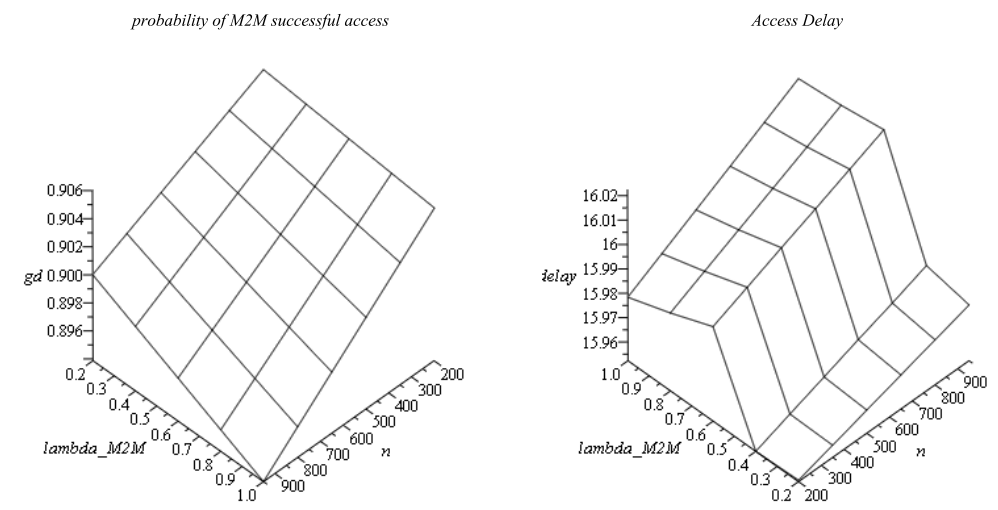

(c) Pro. of success when $\mathrm{H} 2 \mathrm{H}$ arrival(d) Access Delay when $\mathrm{H} 2 \mathrm{H}$ arrival rate is 220 calls/sec rate is 220 calls/sec

Figure 3.18: Performance parameters for VM2M overlay network for $P F=2, N_{M}=8$ $c_{f}=1$

\section{Case 3: High VM2M traffic}

In some scenarios of dense population urban areas like important intersection with lots of vehicles, we might need more dedicated preamble codes for VM2M overlay subnetworks. As a consequence of more preambles dedicated to VM2M overlay network leaves less preambles for $\mathrm{H} 2 \mathrm{H}$ traffic to access the PRACH. So we might need dense cells in urban areas where we could use small cells for overlay and other cell will handle $\mathrm{h} 2 \mathrm{H}$ traffic. We 
evaluate the impact and capacity of VM2M and $\mathrm{H} 2 \mathrm{H}$ traffic with $N_{M}=12$.
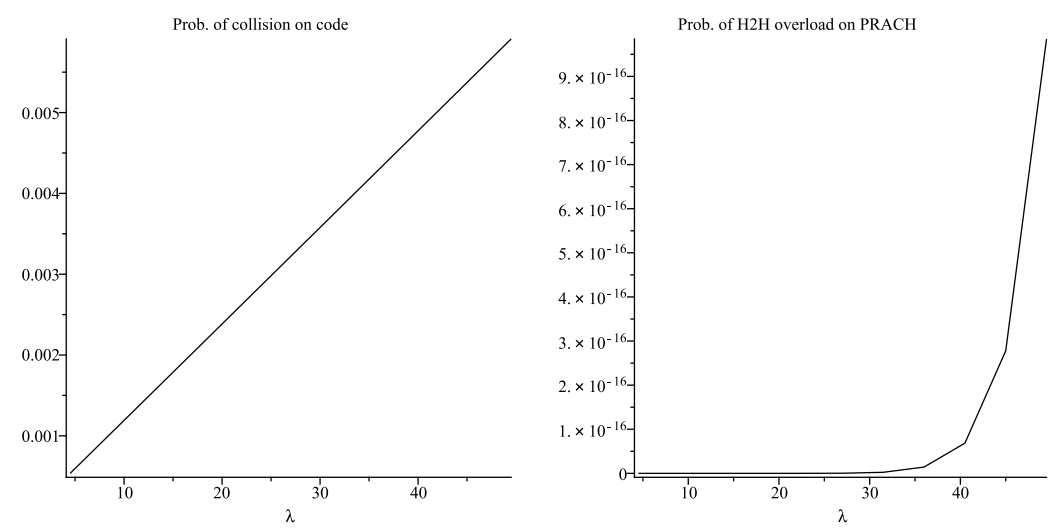

(a) Probability of code collision on (b) Probability of PRACH overload PRACH

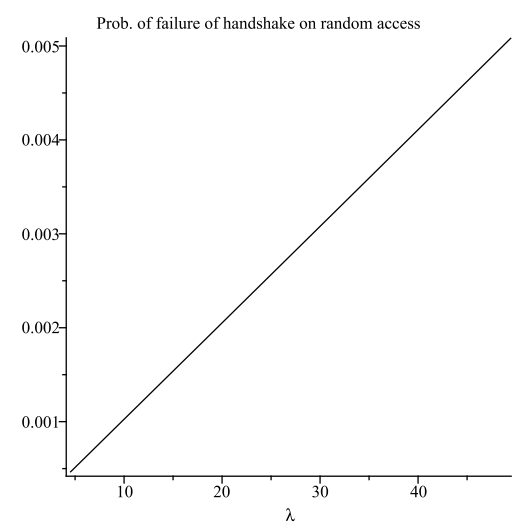

(c) Probability of failure to access on PRACH

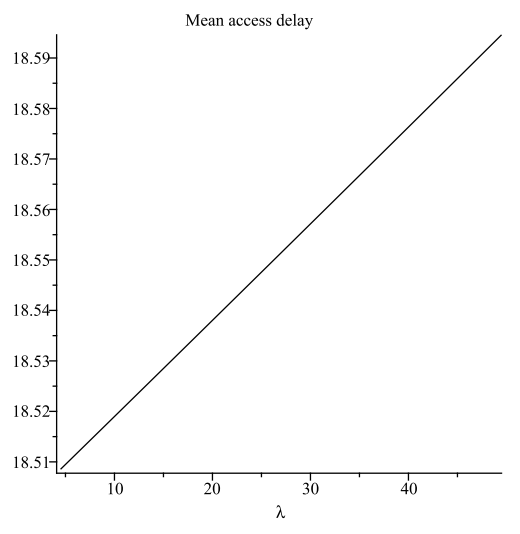

(d) Mean Access Delay

Figure 3.19: Performance of $\mathrm{H} 2 \mathrm{H}$ handshake for $P F=2, N_{M}=12, c_{f}=1$

From Fig. 3.20d, we observe that VM2M probability of success under $\mathrm{H} 2 \mathrm{H}$ arrival rate of 60 calls/sec is $99 \%$ for up to $900 \mathrm{VM} 2 \mathrm{M}$ traffic. We observe that $\mathrm{H} 2 \mathrm{H}$ failure probability ( $3.19 \mathrm{c}$ ) is less than $1 \%$ for up to $60 \mathrm{H} 2 \mathrm{H}$ calls/sec. with very small probability of overload (3.19b). It shows that (Fig. 3.20) we can accommodate more VM2M devices with low collision probability, packet error rate and access delay. 

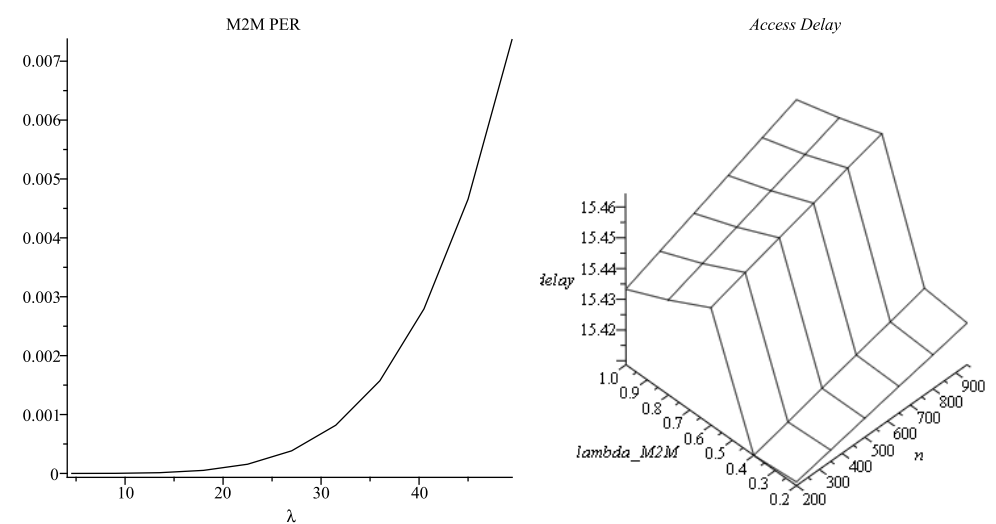

(a) M2M Packet Error Rate (PER)

(b) Access Delay when $\mathrm{H} 2 \mathrm{H}$ arrival rate is 60 calls/sec
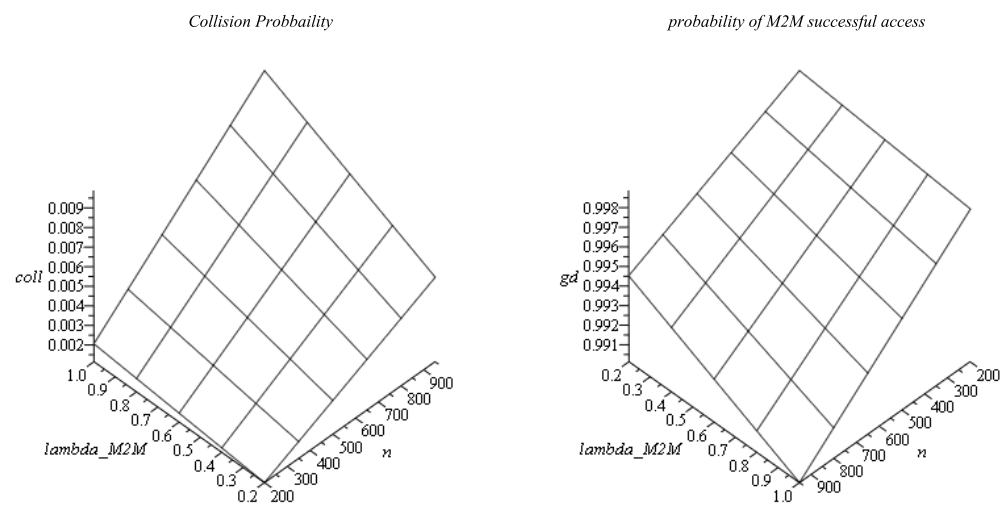

(c) Collision Probability when $\mathrm{H} 2 \mathrm{H}$ ar-(d) Pro. of success when $\mathrm{H} 2 \mathrm{H}$ arrival rival rate is 60 calls/sec rate is 60 calls/sec

Figure 3.20: Performance parameters for VM2M overlay network for $P F=2, N_{M}=12$, $c_{f}=1$

\section{Discussion}

We have presented three deployment scenarios over our proposed overlay networks. All of the combinations of $N_{M}$ are suitable for different situations. If we have low VM2M traffic scenario we can use $N_{M}=4$. We are doing a tradeoff between $\mathrm{H} 2 \mathrm{H}$ and VM2M traffic. In this scenario, we are taking the minimum preamble codes which can handle VM2M traffic. With $N_{M}=4$ we observe that it restricts the capacity of VM2M devices up to 680 with 
$250 \mathrm{H} 2 \mathrm{H}$ calls/sec with $98 \%$ of success probability (Fig. 3.16d). The numbers show the capacity limit of both subnetworks. It restricts the capacity of VM2M networks because of the fact that with 4 preamble codes the capacity of the superframe for format 2 is 51 bytes which allow only maximum 1 packet per frame. The features of IEEE 802.15.4 CSMA/CA are not working and even packets are colliding at the beginning of CSMA/CA. Although assigning only 4 preambles for VM2M overlay increases the maximum capacity for $\mathrm{H} 2 \mathrm{H}$ traffic but it limits the VM2M overlay network to work only on low density scenarios. For moderate traffic scenarios our target is to find the capacity limit for VM2M traffic, where $\mathrm{CCH}$ overlay network could work properly without sacrificing $\mathrm{H} 2 \mathrm{H}$ traffic. It shows the capacity limit for $\mathrm{H} 2 \mathrm{H}$ less than 180 calls/sec with less than $2 \%$ of call failure rate and 900 VM2M devices.

The use of overlay network for the $\mathrm{CCH}$ affects $\mathrm{H} 2 \mathrm{H}$ traffic with the deterministic impact of $N_{M}$ active preambles. On the other hand variable rates of $\mathrm{H} 2 \mathrm{H}$ requests present variable (random) interference to VM2M overlay which is a much bigger challenge. All these are deployment scenarios and we can choose according to situations if we want less capacity for VM2M than we have more capacity for $\mathrm{H} 2 \mathrm{H}$ traffic.

\subsubsection{Experiment 3: Priority based VM2M communications over LTE}

In this experiment, we evaluate the impact of human to human $(\mathrm{H} 2 \mathrm{H})$ layer over vehicular M2M (VM2M) overlay when VM2M gives priority to the physical layer to access the base station (eNodeB). Due to large cell sizes and velocities of vehicles the VM2M has to be implemented using at least format 2 with duration of 2 milliseconds so that it works in urban, suburban, rural areas and highways. We compare the non priority scenarios when 
$\mathrm{H} 2 \mathrm{H}$ and VM2M traffic access the base station simultaneously with the same power, with VM2M priority based scenarios where each preamble of M2M overlay is transmitted with higher power than non-overlay. We evaluate the impact on the performance of VM2M and $\mathrm{H} 2 \mathrm{H}$ traffics

In urban, rural, sub-urban areas and highways with moderate vehicular traffic where both $\mathrm{H} 2 \mathrm{H}$ and VM2M communication traffic intensities are moderate both $\mathrm{H} 2 \mathrm{H}$ and VM2M traffic could work together without interfering each other. However, in several scenarios of urban areas such as important intersections with lots of vehicles, where the vehicle population is dense, continuous $\mathrm{CCH}$ connection is required to transmit safety messages. The VM2M traffic can not be delayed due to $\mathrm{H} 2 \mathrm{H}$ traffic. Delay in real time messages like traffic congestion could make the messages meaningless and the results could be devastating. We implement and evaluate the impact of $\mathrm{H} 2 \mathrm{H}$ and VM2M overlay network by comparing the non priority and priority based scenarios where priority has given to VM2M layer over $\mathrm{H} 2 \mathrm{H}$ to access the base station (eNodeB). In the physical layer of VM2M the preambles are transmitted with higher power than $\mathrm{H} 2 \mathrm{H}$ traffic which gives them priority over $\mathrm{H} 2 \mathrm{H}$ traffic. In moderate traffic of $\mathrm{H} 2 \mathrm{H}$ and VM2M we could implement the scenarios of non priority but to transmit safety messages in a busy intersection the VM2M priority based scenarios is more appropriate to deploy. We have shown VM2M priority based scenarios work well in VANET and allow a fair coexistence of VM2M and $\mathrm{H} 2 \mathrm{H}$ traffics.

In this experiment, the $\mathrm{CCH}$ is implemented as a MAC overlay on PRACH to transmit safety messages. The SCH can be implemented on any other scheduled channels of LTE for transmitting other infotainment messages. The physical layer of overlay network uses dedicated preamble codes and the medium access control layer uses CSMA/CA algorithm 
with 2-way handshaking instead of 4-way handshaking that resembles the one used in the IEEE 802.15.4 [12] standard. The $\mathrm{CCH}$ superframe starts immediately after the reception of a beacon and the messages will be transmitted to eNodeB after completing the backoff countdown. In the first step of handshaking the preambles of overlay networks are transmitted with higher power ratio $\delta=P_{V M 2 M} / P H 2 H$ in the physical layer than $\mathrm{H} 2 \mathrm{H}$ traffic. The power ratio is higher then the transmitted power of $\mathrm{H} 2 \mathrm{H}$ traffic.

\section{Performance of VM2M and H2H Traffic}

In the numerical evaluation, we deploy analytical model from [61] in order to evaluate the capacity and impact of $\mathrm{H} 2 \mathrm{H}$ and VM2M traffic when VM2M traffic were given higher priority to access the $\mathrm{RACH}$ over $\mathrm{H} 2 \mathrm{H}$ traffic. We evaluate the capacity of VM2M overlay with respect to the capacity of $\mathrm{H} 2 \mathrm{H}$ layer with fixed $N_{M}=8$ preamble codes which are permanently active as physical layer of VM2M overlay and intensity of $\mathrm{H} 2 \mathrm{H}$ requests is variable. We evaluate the performance of following experiments for VM2M traffic with non priority and priority based:

$$
P F=2, c_{f}=1
$$

In the priority based scenarios each preamble of VM2M overlay is transmitted with $P_{V M 2 M} / P_{H 2 H}=1 \mathrm{~dB}$, higher power level than non-overlay. In non priority scenarios, preambles from overlay and non-overlay are transmitted with the same power level.

\section{Non priority scenario:}

In the first experiment we evaluate the capacity of both subnetworks with out giving priority to one layer over other to access the base station (eNodeB). Figure 3.21c shows 
Chapter 3: Non priority CSMA/CA overlay network to support vehicular traffic over LTE

$\mathrm{H} 2 \mathrm{H}$ failure probability is $3 \%$ for up to $300 \mathrm{H} 2 \mathrm{H}$ calls/s. If we restrict the $\mathrm{H} 2 \mathrm{H}$ failure probability up to $2 \%$ than with very small overloads ( $3.21 \mathrm{~b}$ ) and $2 \%$ of collision probability ( 3.21a) hints capacity of $\mathrm{H} 2 \mathrm{H}$ traffic to 140 calls/s. Moreover, the capacity of $\mathrm{H} 2 \mathrm{H}$ layer has to limit up to 140 calls/s because beyond that the packet error rate (PER) 3.22b grows fast and impacts failure and collision probability of VM2M overlay. For example, figure 3.22b shows PER of $6 \%$ which increase the failure probability more than $10 \%$ with 300 $\mathrm{H} 2 \mathrm{H}$ calls/s. Figure $3.22 \mathrm{c}$ demonstrates $2 \%$ failure rates for VM2M traffic with 1000 OBUs when the $\mathrm{H} 2 \mathrm{H}$ request rate is 140 calls per second. The collision probability of VM2M traffic (Fig. 3.22d) is $1 \%$ with $140 \mathrm{H} 2 \mathrm{H}$ requests per second. If we restrict the VM2M failure probability to $2 \%$ with small PER the $\mathrm{H} 2 \mathrm{H}$ requests arrival rate of 140 requests per second coincides with the reasonable VM2M capacity of $1000 \mathrm{OBU}$ devices each transmitting one packet per minute. The VM2M traffics has very low SINR violation with $140 \mathrm{H} 2 \mathrm{H}$ calls (Figure 3.22a). The access delay of VM2M subnetworks is $40 \%$ less as compared to the access delay of $\mathrm{H} 2 \mathrm{H}$ networks as shown in Figures $3.21 \mathrm{~d}$ and 3.22e. This is because of the fact $\mathrm{H} 2 \mathrm{H}$ traffic is accessing RACH following 4 way handshaking where as overlay sub network reduces it to 2 way handshaking.

\section{Priority given to VM2M traffic}

In the second experiments, priority has given to physical layer of VM2M overlay over $\mathrm{H} 2 \mathrm{H}$ to access to the base station (eNodeB). We evaluate the impact of $\mathrm{H} 2 \mathrm{H}$ and VM2M layer when VM2M layer given higher priority over H2H. Comparing figure 3.23 with figure 3.21 we demonstrate that the $\mathrm{H} 2 \mathrm{H}$ layer overload probability is more as VM2M traffic given priority over $\mathrm{H} 2 \mathrm{H}$. On the other hand VM2M traffics overload (Fig. 3.24a) is 

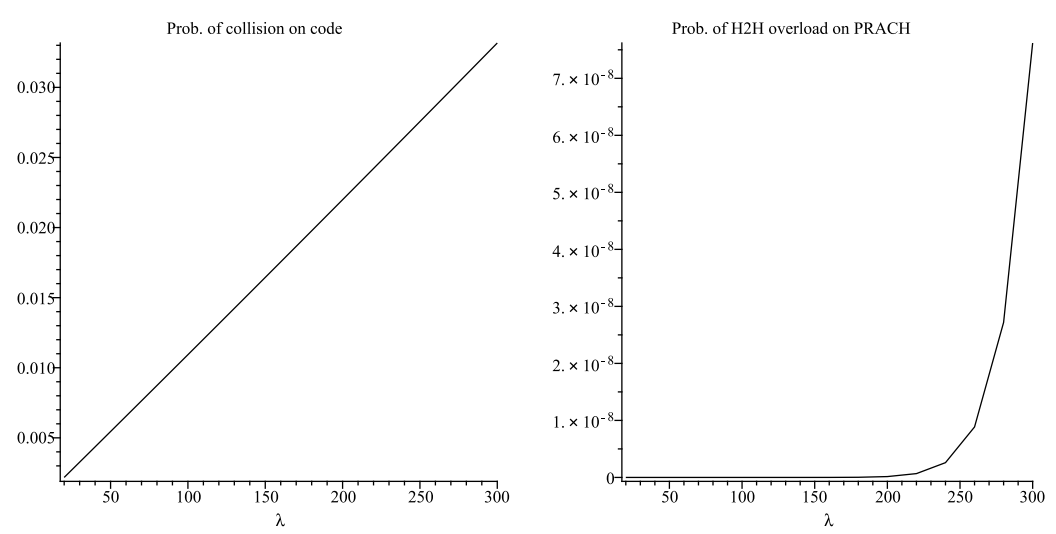

(a) Probability of code collision on

(b) Probability of PRACH overload PRACH

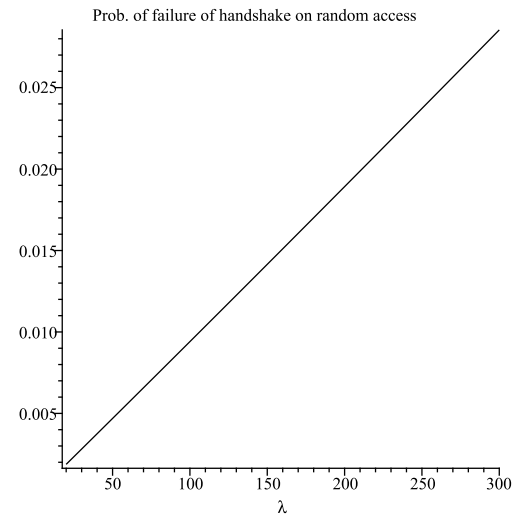

(c) Probability of failure to access on PRACH

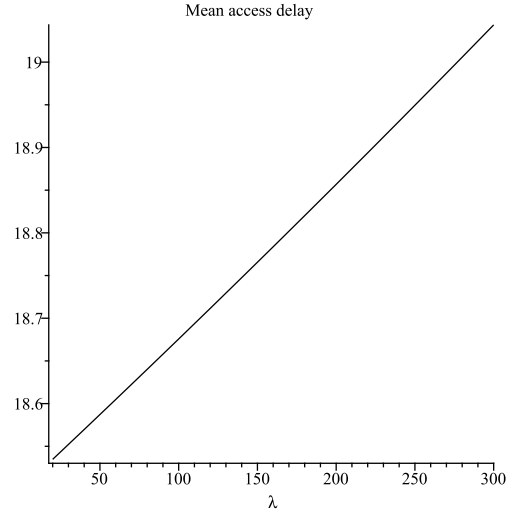

(d) Mean Access Delay in ms

Figure 3.21: Performance of $\mathrm{H} 2 \mathrm{H}$ handshake for $P F=2$ with $c_{f}=1$ without priority.

less compared with $\mathrm{H} 2 \mathrm{H}$ (Fig. 3.21b). If we restrict the PER up to 1\% (Figs. 3.24b) and failure probability up to $2 \%$ (Fig. 3.24c) for VM2M layer than capacity of $\mathrm{H} 2 \mathrm{H}$ layer has to limit up to 220 calls/sec leaving capacity for VM2M OBUs to 1000. Figure 3.23 shows with $220 \mathrm{H} 2 \mathrm{H}$ calls/sec the H2H subnetworks collision (Fig. 3.23a), overload (Fig. 3.23b) and failure probability (Fig. 3.23c) are more compared with non priority scenario. Priority based scenario demonstrate that we could push more H2H traffic (220 calls/s) to suffer 
increases the capacity and ensure smooth operation for 1000 VM2M OBUs each having the traffic of 1 packet per minute as shown in figure 3.24. It demonstrates that VM2M traffics are so strong with priority that with $220 \mathrm{H} 2 \mathrm{H}$ calls/s still we could have 1000 OBUs in the subnetwork. The access delay of VM2M subnetworks is $40 \%$ less as compared to the access delay of $\mathrm{H} 2 \mathrm{H}$ networks as shown in Figures $3.24 \mathrm{e}$ and $3.23 \mathrm{~d}$.

\section{Discussion}

In urban, rural, sub-urban areas and highways with moderate traffic of $\mathrm{H} 2 \mathrm{H}$ and VM2M we could implement the non priority scenarios of with out giving priority to one layer over other. In several scenarios of urban areas such as important intersections with lots of vehicles, where the vehicle population is dense, continuous $\mathrm{CCH}$ connection is required the VM2M priority based scenarios is more appropriate to deploy. This analysis shows that for priority based scenarios, if we restrict the PER up to $1 \%$ as the limit of interference of $\mathrm{H} 2 \mathrm{H}$ traffic over VM2M overlay networks then $220 \mathrm{H} 2 \mathrm{H}$ calls/s still ensure smooth operation for 1000 VM2M OBUs each having the traffic of 1 packet per minute as shown in figure 3.24. On the other hand non priority scenarios restrict the capacity of VM2M to 1000 OBUs for up to $140 \mathrm{H} 2 \mathrm{H}$ calls/s. It is expected that $\mathrm{H} 2 \mathrm{H}$ traffic will be affected when VM2M traffic given higher priority to access the base station. In busy intersections with lots of vehicles scenarios, figure 3.23 show with 220 calls/sec $\mathrm{H} 2 \mathrm{H}$ traffic ensure smooth operation with high probability of success by leaving more capacity for VM2M traffic. The priority based scenario demonstrates that with more $\mathrm{H} 2 \mathrm{H}$ calls compared with non priority scenarios we could still ensure smooth operation of VM2M traffic. We evaluate the impact and capacity of both subnetworks and observed that depends on the deployment situations both scenarios 
could perform better. We wanted to maximize the capacity of VM2M but can not sacrifice the $\mathrm{H} 2 \mathrm{H}$ traffic greatly. On the other hand, it is a great challenge to handle the variable rates of $\mathrm{H} 2 \mathrm{H}$ request as it causes variable (random) interference to VM2M overlay.

\subsection{Chapter summary}

In this chapter, we consider VANET as a case study for smart city application areas. We propose a Vehicular M2M (VM2M) overlay network over random access channel (RACH) in LTE that aims to emulate the control channel $(\mathrm{CCH})$ of vehicular ad hoc networks (VANETs). VM2M overlay is implemented over a dedicated subset of preamble codes, at the physical layer, and uses a medium access control (MAC) layer modelled as IEEE 802.15.4 carrier sense multiple access (CSMA/CA) mechanism. VM2M devices avoid the complexity of the four-way handshake, which reduces the interference on the PRACH both to and from $\mathrm{H} 2 \mathrm{H}$ devices, in the same cell and in neighboring cells. To the best of our knowledge, this approach has not been proposed or discussed elsewhere. We evaluate the performance of the proposed scheme through a detailed analytical approach and investigate the interaction of regular LTE $(\mathrm{H} 2 \mathrm{H})$ traffic and VM2M traffic. We conduct 3 experiments to find the feasible combinations of $\mathrm{RACH}$ resource configuration and preamble format (PF) in large cells and to find the cell capacity for $\mathrm{H} 2 \mathrm{H}$ and M2M users.

We have found that the choice of preamble format for $\mathrm{H} 2 \mathrm{H}$ and VM2M depends on the environment. For sub-urban, rural and highway scenarios RACH format 2 (or even 3) might be necessary due to large velocity and round trip-time. Use of format 2 increases the power budget and gives the priority to $\mathrm{H} 2 \mathrm{H}$ traffic since each preamble is transmitted twice. For urban environment RACH format 0 can be used and capacity can be increased with 
increasing $\mathrm{RACH}$ configuration parameter. The use of overlay network for the $\mathrm{CCH}$ affects $\mathrm{H} 2 \mathrm{H}$ traffic with the deterministic impact of $N_{M}$ active preambles. On the other hand variable rates of $\mathrm{H} 2 \mathrm{H}$ requests present variable (random) interference to VM2M overlay which is a much bigger challenge. The capacity of M2M overlay can be increased by increasing the number of preambles $N_{M}$ used in the VM2M physical layer. This is also beneficial from the aspect of CSMA/CA since superframe capacity will increase and packet access can be spread more evenly over the superframe duration. We evaluate the impact and capacity of both subnetworks and observed that depends on the deployment situations both scenarios could perform better. We wanted to maximize the capacity of VM2M but can not sacrifice the $\mathrm{H} 2 \mathrm{H}$ traffic greatly. On the other hand, it is a great challenge to handle the variable rates of $\mathrm{H} 2 \mathrm{H}$ request as it causes variable (random) interference to VM2M overlay. We have shown that with $N_{M}=8$ dedicated preamble codes for VM2M overlay, both LTE and VM2M networks can have fair coexistence. In the scenarios of transmitting safety messages of VM2M with higher power, we evaluate the impact of human to human $(\mathrm{H} 2 \mathrm{H})$ layer over VM2M overlay when VM2M gives priority to the physical layer to access the base station (eNodeB). We showed that VM2M traffics are so strong with priority that with $220 \mathrm{H} 2 \mathrm{H}$ calls/s still, we could have 1000 OBUs in the subnetwork. 

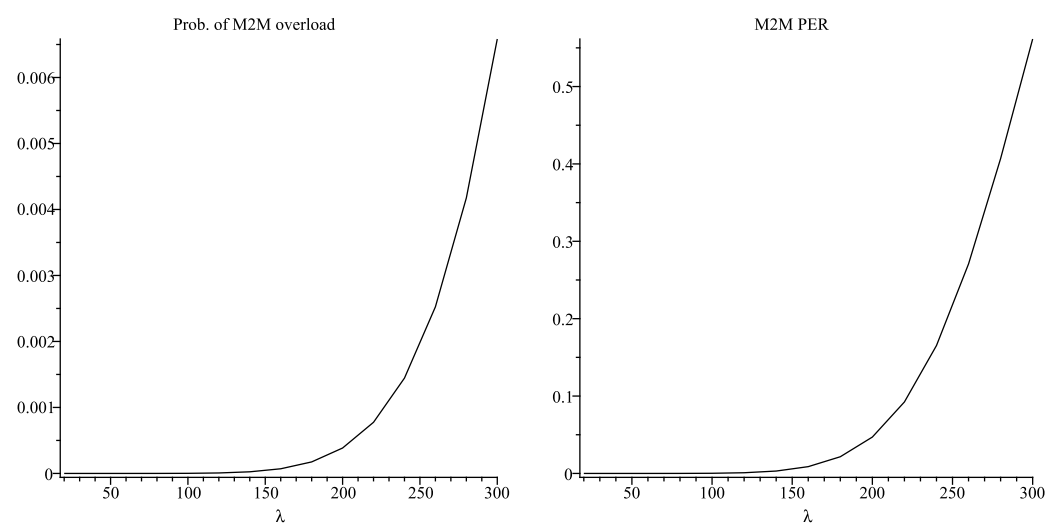

(a) Probability of M2M overload on

(b) M2M Packet Error Rate (PER) PRACH caused by $\mathrm{H} 2 \mathrm{H}$ traffic
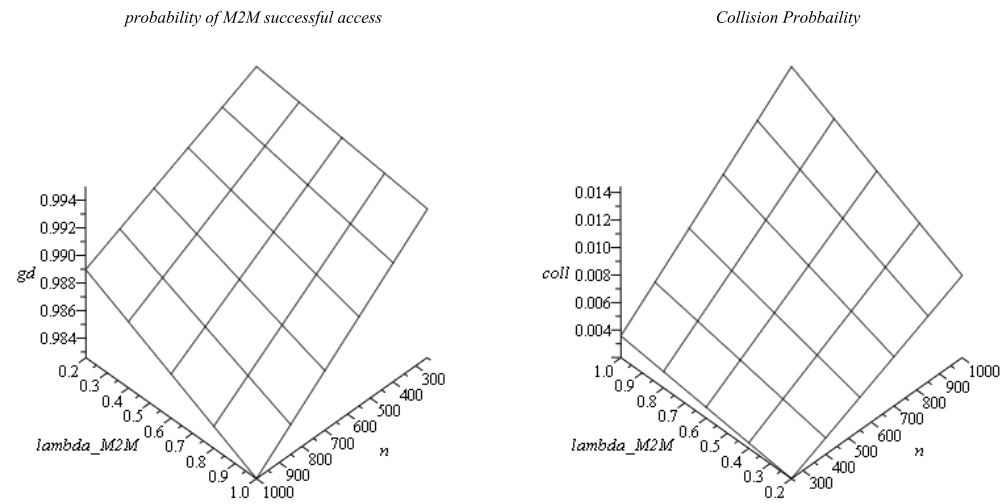

(c) Probability of M2M successful ac-(d) M2M Collision Probability when cess on $\mathrm{PRACH}$ when $\mathrm{H} 2 \mathrm{H}$ arrival rateH $2 \mathrm{H}$ arrival rate is 140 calls/sec is 140 calls/sec

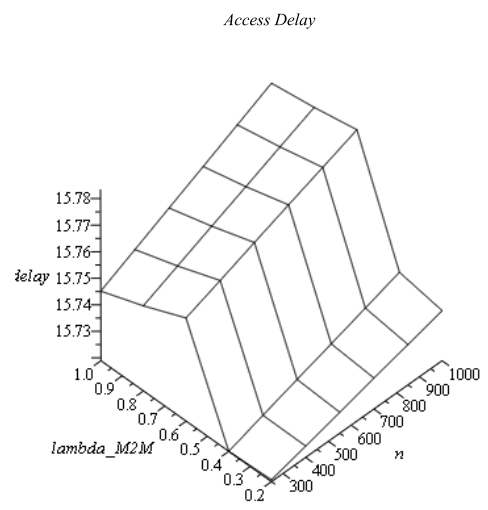

(e) M2M Access Delay when $\mathrm{H} 2 \mathrm{H}$ arrival rate is 140 calls.sec

Figure 3.22: Performance parameters for M2M overlay network for $P F=2$ and $c_{f}=1$ without priority 

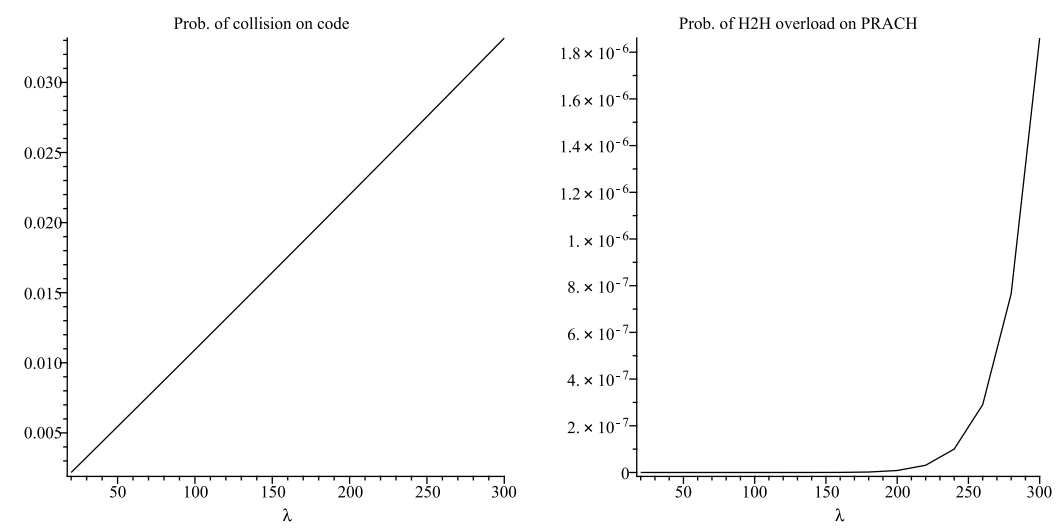

(a) Code Collision Probability of (b) Probability of PRACH Overload PRACH

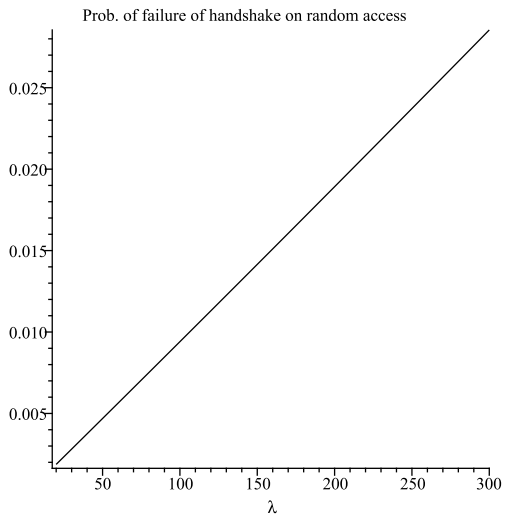

(c) Failure Probability to Access PRACH

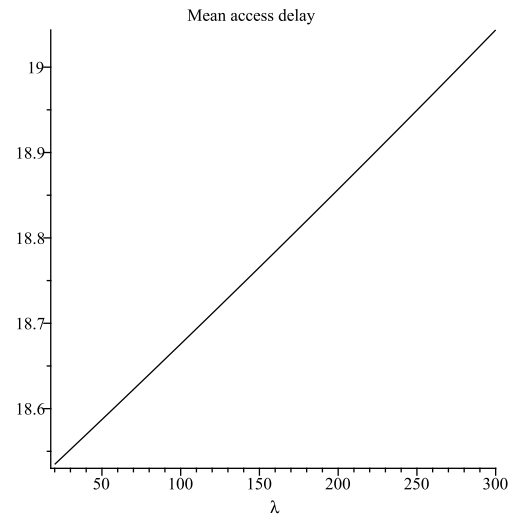

(d) Mean Access Delay

Figure 3.23: Performance of $\mathrm{H} 2 \mathrm{H}$ Handshake for $P F=2, N_{M}=8 c_{f}=1$ with VM2M priority 


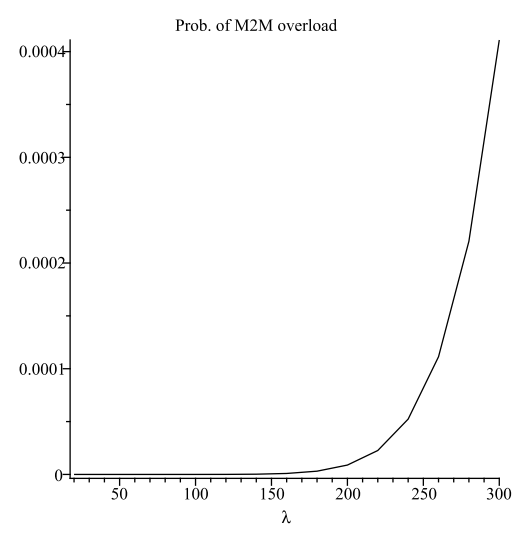

(a) Probability of M2M overload on PRACH caused by $\mathrm{H} 2 \mathrm{H}$ traffic

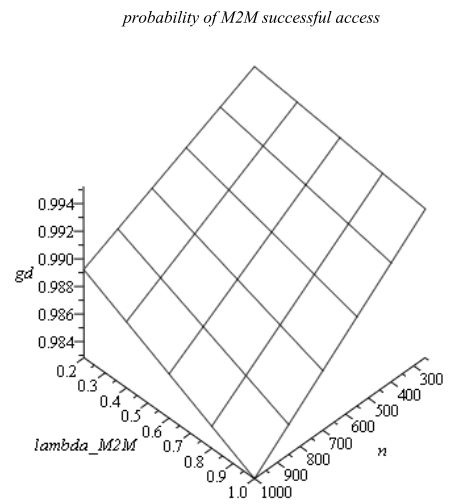

(c) Success Probability when $\mathrm{H} 2 \mathrm{H}$ Ar-(d) Collision Probability when $\mathrm{H} 2 \mathrm{H}$ rival Rate is 220 calls/sec

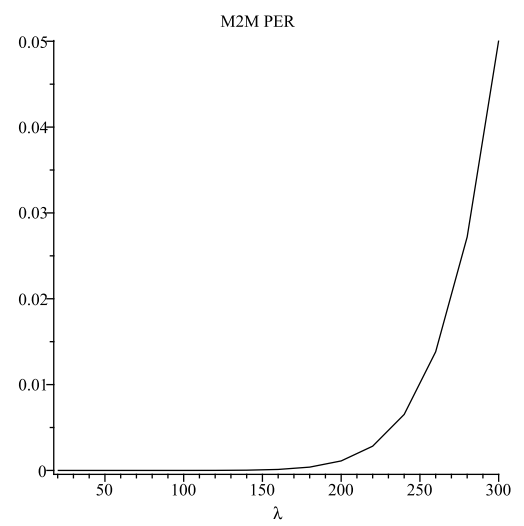

(b) M2M Packet Error Rate (PER)

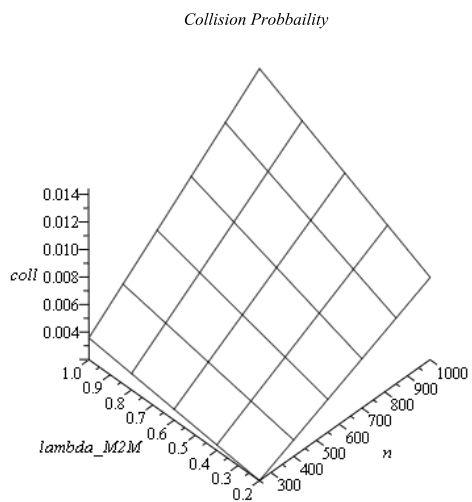

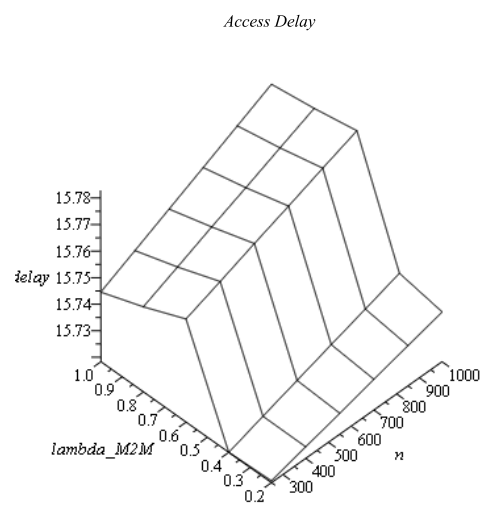

(e) M2M Access Delay when $\mathrm{H} 2 \mathrm{H}$ Arrival Rate is 220 calls/sec

Figure 3.24: Performance of VM2M Overlay Network for $P F=2, N_{M}=8$ and $c_{f}=1$ with VM2M priority 


\section{Chapter 4}

\section{Priority based CSMA/CA overlay}

\section{network over LTE}

In chapter 3 we consider VANET as a case study for the smart city application area of smart transportation system. We propose a non-priority CSMA/CA overlay network (VM2M) wherein the MAC layer we consider CSMA/CA similar to the one used in IEEE 802.15.4 as the transmission control protocol. IEEE 802.15.4 is a low rate, low cost, low power, short range wireless communication personal area network. It does not support high data rate communications and does not provide priority among different traffic classes. In smart city scenarios among different traffic classes, for example, vehicular emergency messages should have higher priority over crowd sensing. We want to deploy priority among messages. IEEE 802.15.6 supports high data rates and define priorities among different traffic classes through access categories. These features of IEEE 802.15.6 have motivated us to consider this standard to deploy in our proposed priority based CSMA/CA (PM2M) overlay network for smart city application areas. 
In this chapter, we propose a priority based CSMA/CA machine to machine (PM2M) overlay network over LTE to transmit data with particular attention to the areas of, low transfer latency and reliability. The proposed overlay network is applicable in the M2M application areas to build smart cities specifically for smart transportation systems, crowd sensing, smart health care system (e-health), smart parking and smart street lighting. The proposed architecture uses LTE physical random access channel (PRACH) as the underlying transport for a PM2M overlay network. The architecture is implemented using a dedicated subset of preambles at the physical layer, and a carrier sense multiple access with collision avoidance (CSMA/CA) mechanism similar to the one used in IEEE 802.15.6 at the medium access control layer. We evaluate the performance of overlay network with and without consideration of noise errors coming from the physical layer of $\mathrm{H} 2 \mathrm{H}$ traffic and compare the different performance metrics like the probability of success, throughput, mean backoff time and the number of backoff attempts. We compare our proposed approach with a reference approach [87] and shows that our approach outperforms with respect to the probability of success and access delay.

\subsection{Introduction}

Increasing growth of machine to machine (M2M) communications will lead to allowing build a smart city in reality. In smart city scenarios application areas, such as VANET, crowd sensing, healthcare data reporting, smart parking and smart street lighting we have coverage with WiFi but WiFi goes through different ISPs and it is suitable for short-range communications. It is insufficient for large communication range with high data rates and suffers from interference by surrounding networks. For VANETs we have IEEE 802.11p 
standard which enables V2V and V2R communications through the on-board unit (OBUs) which is vehicles interface to VANET. However, the standard is not well suited for the vehicular machine to machine communications due to unbounded delay, limited radio range, low scalability, and lack of persistent infrastructure [7]. In some scenarios of dense population urban areas like an important intersection with lots of vehicles or medical healthcare data reporting to the remote server, emergency messages need to transmit without any delay with higher priority and in real time. In case of multiple emergency events, the nodes cannot transmit the data within the defined superframe time and data may be lost due to overload and collisions. We have to adopt a suitable wireless communication network and also a priority based transmission control protocol that will categorize all the traffic classes according to user priorities. We also have to design an efficient overload control mechanism to alleviate the overload problem of the access network.

IEEE 802.15.6 is a low power, short range, priority based highly reliable wireless communication systems. IEEE 802.15.6 supports large data rates and define priorities among different traffic classes through access categories. The standard is designed to support advanced medical and entertainment options [69]. It is not only dedicated to medical and health applications, it is also designed to support consumer electronics, sports, military applications and advanced medical equipment which enables the consumer to have power efficient, reliable and interference free solutions to deploy a variety range of devices [20]. The standard supports different data rates for different applications and up to $10 \mathrm{Mbps}$ data rate is required. IEEE 802.15.6 provides necessary functionality to give priorities to emergency messages through contention window $(\mathrm{CW})$ sizes and also the time duration over which it can transmit in a super frame [35]. These features of IEEE 802.15.6 have moti- 
vated us to consider this standard to deploy in our proposed overlay architecture for smart city application scenarios.

In a smart city, besides WiFi, we also have ubiquitous cellular coverage and we can share some resources like preambles to implement overlay networks which are affordable and cheap and can be shared by community networks like VANET, crowd sensing, healthcare reporting. For example, if we want to send crowd sensing information it will go through the interface over these shared preambles. Long-Term Evolution (LTE) or 4G LTE, is a standard for cellular wireless communication which can offer solutions over higher distances and speeds. Its high data rate, low latency and large coverage features give LTE advantages over other technologies. While designing an overlay network we need to consider two important factors, data transfer latency should be as small as possible and data should be transferred reliably. LTE supports reliable data transmission with high data rates for real safety messages over large coverage areas. LTE established a connection with base stations (eNodeB) over physical random access channel (PRACH) through four-way handshaking. LTE accommodates wireless random access with 4 different preamble formats (PF) which differ by the size of PRACH resource, cyclic prefix time (CP) and guard time (GT) [74]. Random access in large cells with large propagation delays is supported with $\mathrm{PF}=2$ where two consecutive LTE subframes are allocated to PRACH resource. On the other hand, PF $=0$ could be used for the large density of data and relatively small cell size.

All of the above features have motivated us to consider the possibility of using LTE random access channel as overlay network for M2M communications. LTE is better suited to transmit the higher volume of data with high data rate and timely fashion. In this paper, we propose a novel priority based machine to machine (PM2M) overlay network over LTE 
random access channel (RACH) which will work parallel with human to human traffic. To transfer data from nodes (vehicles or sensors) to LTE base station (eNodeB) PM2M uses dedicated preamble set at the physical layer and carrier sense multiple access with collision avoidance (CSMA/CA) as the medium access control (MAC) protocol. This MAC layer resembles the one used in IEEE 802.15.6. The challenge is to ensure that PM2M traffic and $\mathrm{H} 2 \mathrm{H}$ traffic have fair access to the $\mathrm{RACH}$ resources. We developed an analytical model with the error coming from the physical layer of $\mathrm{H} 2 \mathrm{H}$ traffic, composed of a 3 dimensional Markov chain in order to model the CSMA/CA procedure of IEEE 802.15.6. We investigate the effect of error in our physical and MAC layer with the presence of $\mathrm{H} 2 \mathrm{H}$ traffic. Our approach also provides the ability to calculate the mean number of backoff attempts in every backoff stage. An extensive number of results has been produced in order to validate the proposed novel model. The results are produced by solving the numerical calculations of the analytical models in Maple [56]. We analyze the performance of joint M2M and H2H system and investigate tradeoffs needed to keep satisfactory performance and reliability for M2M traffic in the presence of $\mathrm{H} 2 \mathrm{H}$ traffic of known intensity. We investigated the capacity of PM2M overlay networks with and without consideration of error and we have shown that overlay network allows fair coexistence of PM2M and $\mathrm{H} 2 \mathrm{H}$ traffic. To validate our proposed method, we also compare our approach with a reference approach [87] and prove that our approach outperforms than the reference approach. Our results confirm the validity of this approach for applications in crowd sensing, monitoring, and others utilized in smart city development. 


\subsection{PRACH architecture and random access procedure}

The PRACH is an interface between non-synchronized user equipment (UE, the LTE term for a terminal device) and the orthogonal transmission scheme of the LTE uplink radio access. To established an uplink transmission a UE has to scheduled and synchronized with the current cell through a request sent on the PRACH. A UE node needs to sent request to PRACH if it is not connected and request for initial access when it is connected but not uplink synchronized to send uplink data or to receive downlink data and also when it performs handover calls. The evolved NodeB (eNodeB, the LTE base station) refers the request as random access and schedule orthogonal uplink transmission resources for communications to and from the UE.

Available bandwidth resources for LTE cell are divided in time and frequency domain matrix. Time access is organized in frame that lasts $10 \mathrm{~ms}$ and consists of 10 subframes with duration of $1 \mathrm{~ms}$ each. Subframes can be divided into two $0.5 \mathrm{~ms}$ slots. In the frequency domain resources are grouped in units of 12 OFDM subcarrier with a total bandwidth of $180 \mathrm{kHz}$. Basic access unit either for random or scheduled access is resource block (RB) which consists of 12 sub carriers over one subframe duration of $1 \mathrm{~ms}$. For control channels, finer granularity is available during one slot. Smallest resource unit for control channels is resource element (RE) and consists of one sub carrier for the duration of one OFDM symbol. Cell bandwidth can be configured in FDD or TDD. According to time division duplex (TDD) unpaired spectrum frame structure type 2 subframes 0 and 5 is always reserved for downlink transmission and other subframes can be used for uplink or downlink transmission. Minimum PRACH bandwidth is composed of six resource blocks in frequency with a bandwidth of $1.080 \mathrm{MHz}$ and one subframe over two frame time. $\mathrm{RACH}$ resources are 
shared by all user equipment, which results in congestion in the network and shortage of resources. Adaptation to larger random access traffic is possible through $16 \mathrm{PRACH}$ configurations as shown in Fig. 4.1. For low traffic intensity and small system bandwidth, one PRACH resource over 2 frames may be sufficient (TDMA configurations 0,1,2 and 15). As traffic increases PRACH resource occurs once per frame (TDMA configurations 3, 4, and 5). Further, as traffic increases PRACH resource can occur once per 5 subframes (TDMA configurations 6,7 and 8) or once per 3 subframes (TDMA configurations 9, 10 and 11). Although previous PRACH allocations avoided interference at granularity of 3 cells for high traffic dense PRACH allocations bring possibility of interference since PRACH resource occurs on every second subframe (configurations 12 and 13) or on every subframe in a frame (configuration 14). However, care must be taken to avoid a possibility of interference between PRACH and PUSCH channels among cells at close distances.

\subsubsection{Random access procedure}

In LTE, UE or mobile terminals (MT) which does not have allocated uplink radio resources perform random access (RA) procedure to connect to the network before they transmit data. Communications in LTE are established over physical random access channel (PRACH). Each node wishing to communicate with base station needs to complete fourway handshake which starts by transmission of preamble. The RA procedure is performed through the RACH [53] and consists of four-way handshaking with the eNodeB as shown in Figure 4.2.

The RA can be contention based or contention free (with reserved 10 preambles for time-critical situation like handover). The contention-based 4-way handshaking procedure 


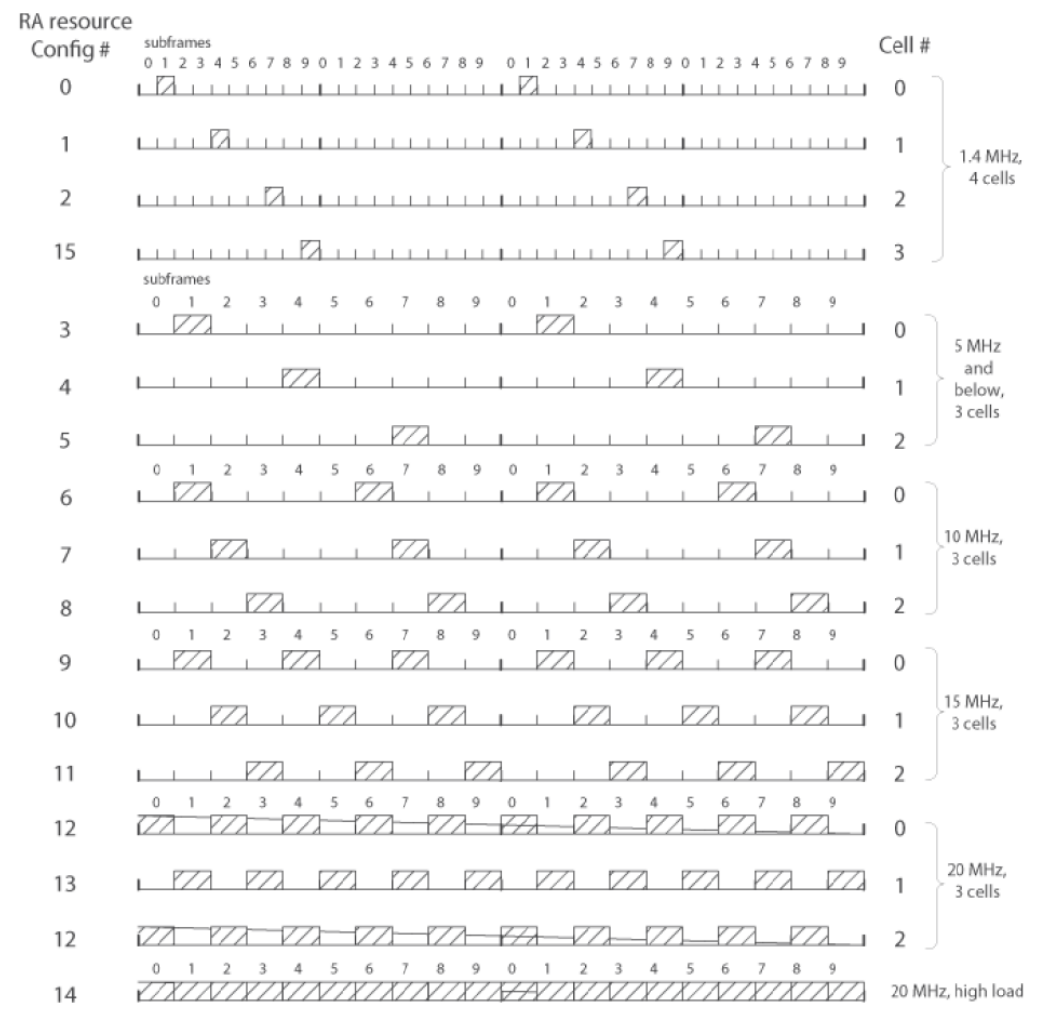

Figure 4.1: PRACH resource configurations, after [74]

of RA contains following steps:

Step 1: The MT randomly selects the preamble from available 54 preamble sequences (among 64, 10 preambles are reserved for handover calls) and transmits it over PRACH to eNodeB.

Step 2: The eNodeB transmits an RA response (RAR) to MT through the downlink shared channel (PDSCH). RAR contains temporary Cell Radio Network Temporary Identifier (CRNTI) identity information. It also contains scheduling information for the third step.

Step 3: Then, MT sends its CRNTI and scheduling information to eNodeB through the 


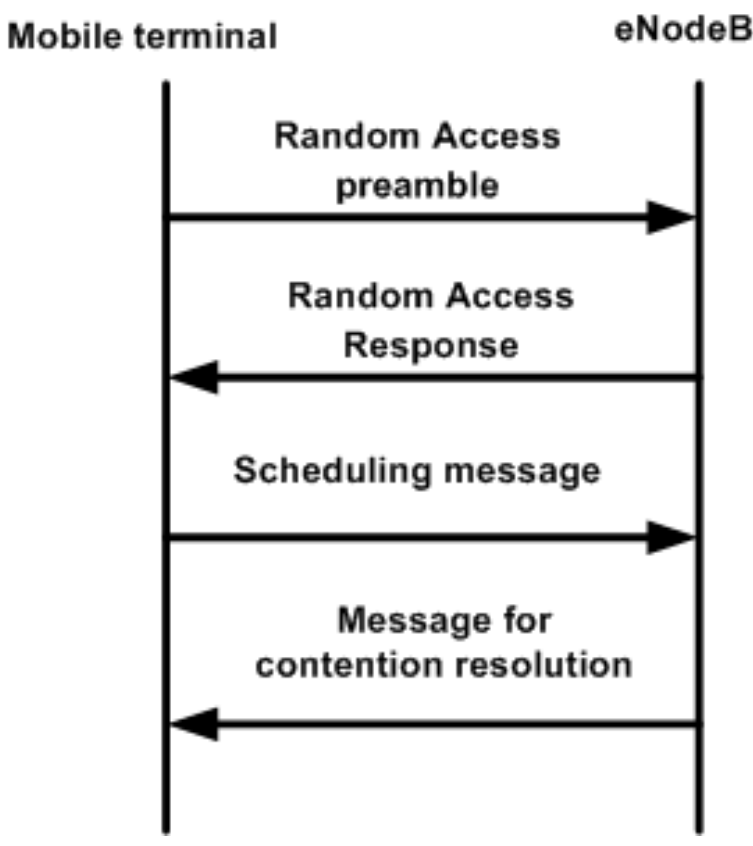

Figure 4.2: Four way handshaking between MT terminal and eNodeB according to [53] physical uplink shared channel (PUSCH) radio resources assigned in step 2.

Step 4: Finally, the eNodeB responds with the confirmation of the identity of MT and finishes the contention procedure.

Unfortunately, first and third steps of handshaking are prone to collisions and overload conditions due to a limited number of preambles. This prevents completion of RA handshake [61] for a large number of short data transmissions from PM2M where SINR (Signal to interference noise ratio) at $\mathrm{eNodeB}$ is violated. In a nutshell use of four-way handshaking procedure of LTE for massive network access is inefficient and leads to network overload. This has motivated our research efforts towards PM2M overlay architecture where PM2M is implemented as an overlay in PRACH. PM2M overlay reduces four step handshaking by two way handshaking to control the overload of the network. 


\subsection{PM2M overlay network over PRACH}

The superframe structure of the proposed PM2M overlay network is shown in figure 4.3 that supports transfer of data from nodes to LTE base station and then data is transmitted to the remote servers. Due to long distances and high volume of data the proposed PM2M has to be implemented using at least format 2 with subframe duration of $2 \mathrm{~ms}$. Superframe will start immediately after the reception of beacon and after completing the backoff (as in IEEE 802.15.6 CSMA/CA) the message will be transmitted to eNodeB. eNodeB has to acknowledge the message, otherwise, message will be retransmitted until the retransmission limit is reached. Other $\mathrm{H} 2 \mathrm{H}$ traffic will follow the 4-way handshaking mechanism to access the network. PM2M overlay network reduces 4 way handshaking to 2 -way procedure.

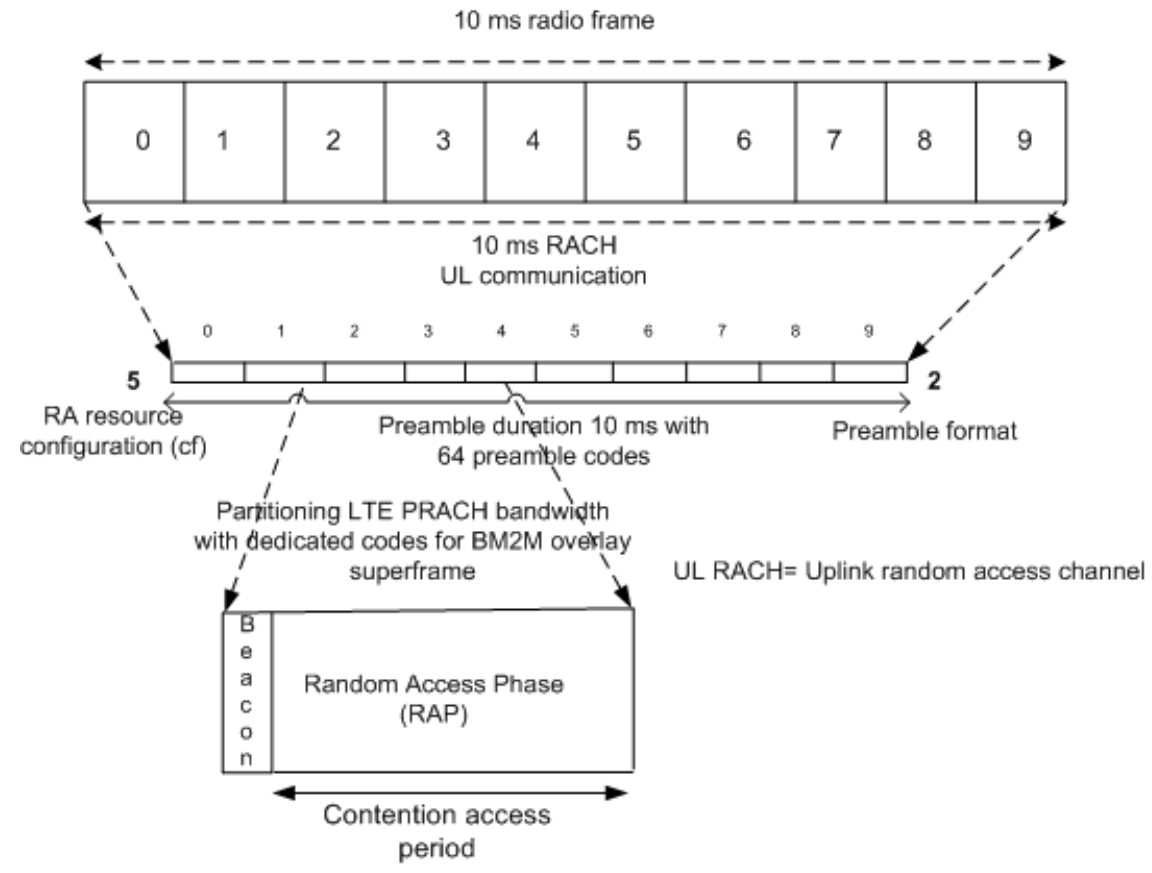

Figure 4.3: LTE and PM2M Overlay Superframe with PF 2 in TDD Mode (2 ms Subframe). 


\subsubsection{PM2M random access procedure}

We propose a two-way handshaking mechanism to control the overload situation of the RA procedure where M2M devices only use the dedicated preambles to send their data packets.

Step 1: In the first step the M2M devices send its data by using the available dedicated preambles. As we separate the resources between $\mathrm{H} 2 \mathrm{H}$ and M2M, M2M devices will not use other preambles allocated for $\mathrm{H} 2 \mathrm{H}$ traffic. M2M devices will compete to get access to PRACH following CSMA/CA mechanism like the one used in IEEE 802.15.6. and in parallel with $\mathrm{H} 2 \mathrm{H}$.

Step 2: After transmitting the data packet the M2M device will wait for the acknowledgement. If it does not receive the acknowledgement due to collision it will retransmit until the maximum retransmission limits.

\subsubsection{Physical and MAC layer}

We deploy analytical model from [72]. We need to adopt a model for IEEE 802.15.6 since our proposed architecture's physical layer is shared with H2H LTE communication on PRACH. We modify the adopted proposed model of [72] because we have to model the probability of error for our sophisticated physical layer. The physical layer of our proposed architecture is implemented with $N_{M}$ dedicated preamble codes from available $N$ codes to access PRACH. This allows PM2M traffic to access the network concurrently with $\mathrm{H} 2 \mathrm{H}$ traffic but with less contention. $N_{M}$ preambles are permanently active as the physical layer of PM2M overlay. With the presence of $\mathrm{H} 2 \mathrm{H}$ traffic, the physical layer is affected and 
our medium access control layer is suffering from errors. We modified the frame time slot according to compatible with the proposed architecture and modified other parameters accordingly. In this work we consider LTE system with 5MHz bandwidth. Bandwidth of PRACH channel is $W=1.08 M H z$. Cell has $N=N_{h}+N_{i}+N_{M}$ orthogonal Zadoff Chu preambles with $N_{h}$ preambles allocated in non contention manner to handoff call, $N_{i}$ codes allocated for $\mathrm{H} 2 \mathrm{H}$ traffic in contention mode and $N_{M}$ codes allocated to PM2M traffic. Typical number of codes per cell is $\mathrm{N}=64$ and we will assume $N_{h}=10, N_{M}=8$ and $N_{i}=46$. PM2M codes are bundled together such that each code carries one bit of each users byte. PM2M access is configured as an CSMA/CA overlay over physical layer which consists of 8 codes. As an overlay PM2M superframe can be configured over $N_{M}$ codes. To transmit a single PM2M data bit, $N_{b}$ preamble elements are used as a kind of chipping sequence, the size of which affects the performance as signal-to-interference- plus-noise ratio (SINR) increases by $10 \log _{10} N_{b}$. We note that correct detection of PM2M data bits requires higher SINR since an $\mathrm{H} 2 \mathrm{H}$ preamble is detected based on the SINR over its entire duration, while only a portion of that duration is used by PM2M traffic.

In this scheme, each PRACH resource holds a PM2M superframe, which makes CSMA/CA access possible. For the medium access control (MAC) layer PM2M access is configured as a CSMA/CA overlay over physical layer which consists of $N_{M}=8$ dedicated preambles. Time for preamble transmission becomes superframe time for PM2M overlay network. The super frame time slot is divided in to beacon period and each consist of equal lengths. We consider the beacon mode with beacon period boundaries where at the beginning of every superframe a beacon is transmitted on the medium. The super frame is divided into Access Phase (AP) and in our model, we only consider random access phase 
1 (RAP1) and all other access phases have zero length. The standard allows to consider other access phase length to zero while, RAP1 should be non-zero. In our model we did not consider RTS/CTS (like in IEEE 802.11) and also 4-way handshaking mechanism of LTE for the overlay PM2M networks as they will create a heavy overload in the PRACH.

CSMA/CA access in superframe resembles the one used in beacon-enabled IEEE 802.15.6. In CSMA/CA overlay one backoff period has 20 sequence elements i.e: tboff $=$ $20 / R=18.51 \mu$ s. We consider preamble format 2 where preamble duration is $1.6 \mathrm{~ms}$ respectively (without cycle prefix and guard time periods) giving preamble element rate as $\mathrm{R}=1.048 \mathrm{M}$ elements per second. Access in format 2 is achieved by repeating the same preamble twice. The number of bits in preamble is 839 and it is repeating in the second subframe. The preamble data rate is $R=1.048 \mathrm{Mbps}$. We assume configuration index $c_{f}=5$ which means that surrounding cells may use the same resource block for PUSCH channel which can cause interference.

We evaluate the performance of PM2M overlay network in the presence of backoff error in CSMA/CA mechanism while accessing the PRACH. In IEEE 802.15.6 during the medium access mechanism through CSMA/CA a node sense the medium while decrementing the backoff counter. If the medium is sensed busy, it freezes the backoff counter and while medium sense idle again it decrements the backoff counter. $\mathrm{H} 2 \mathrm{H}$ traffic is also transmitting in parallel with the PM2M traffic then there is a possibility that we might not listen the medium right over the backoff period. We could hear noise because of the $\mathrm{H} 2 \mathrm{H}$ traffic. Maybe medium is idle but because of the activity we may hear it as busy also when medium is busy maybe we hear it idle. Bit error rate (BER) coming from the $\mathrm{H} 2 \mathrm{H}$ layer has a great impact on the PM2M layer. BER is the probability of hearing one bit wrong. Be- 
fore accessing the medium nodes sense the status of the medium in backoff period, where 1 backoff period is equivalent to 20 bit times. We have 20 bits and listening of each bit can be wrong from the probability of BER. If all of them or more than half of the bits are wrong we have the wrong listening decision which actually depends on the hardware of the networks. In our case, we define the backoff error as the probability that 11 bits are wrong and less than 11 bits are correct. We modified the Markov chain that was proposed in [72] and develop a 3 dimensional Discrete Time Markov chain (DTMCs) in order to model the procedure of CSMA/CA mechanism with backoff error.

\subsubsection{CSMA/CA mechanism}

The PM2M overlay superframe starts immediately after receiving the beacon from eNodeB. In CSMA/CA, before transmitting a frame a device senses the medium and transmits a frame only if the medium is idle. If the medium is busy, the device has to wait for a predefined amount of time until the medium becomes idle. This protocol could avoid collisions as frame is transmitted only when the channel is idle. The CSMA protocol uses clear channel assessment (CCA) as the carrier sensing mechanism. At the beginning of access according to user priorities, the nodes shall calculate the backoff counter (BC) and contention window $(\mathrm{CW})$ to determine when it obtains the access to medium. The user priorities are differentiated by the values of the minimum and maximum CWs. The BC is calculated as an integer random variable uniformly distributed over the interval $[1, \mathrm{CW}]$. If the node sense medium is idle for SIFS amount of time it start decrementing the BC. Any time it senses medium is busy it locks the $\mathrm{BC}$ and wait until the current transmission ends or medium becomes idle again. If the current superframe does not have enough 
time to complete the transmission it locks the $\mathrm{BC}$ and unlocks it when the medium is idle and has enough time to complete the transmissions. After completing the transmission the $\mathrm{CW}$ value is set to minimum. In case of $m_{t h}$ number of unsuccessful transmission the $\mathrm{CW}$ value remains same otherwise $\mathrm{CW}$ is doubled, where $\mathrm{m}$ is an odd number. The $\mathrm{BC}$ is decremented by one for each idle CSMA slot and after backoff counter reaches 0 it gets the chance to send the packets. Otherwise, the device has to wait for the next superframe.

\subsubsection{Priority mapping}

IEEE 802.15.6 provides priorities to emergency messages through contention window (CW) and access categories (AC). There are 8 different $\mathrm{AC}$ which defines user priorities (UP) to access the medium. The CA's are differentiated by the maximum and minimum $\mathrm{CW}$ values. The AC's with the $C W$ values are depicted in table 4.1.

Table 4.1: IEEE 802.15.6 access categories (AC) with user priorities(UP) and contention window $(\mathrm{CW})$ values

\begin{tabular}{|c|c|c|c|}
\hline UP & Traffic & $C W_{\min }$ & $C W_{\max }$ \\
\hline 0 & Background(BK) & 16 & 64 \\
\hline 1 & Best effort(BE) & 16 & 32 \\
\hline 2 & Excellent effort (EF) & 8 & 32 \\
\hline 3 & Controlled load(CL) & 8 & 16 \\
\hline 4 & Video(VI) & 4 & 16 \\
\hline 5 & Voice & 4 & 8 \\
\hline 6 & Network control & 2 & 8 \\
\hline 7 & Emergency or medical event data & 1 & 4 \\
\hline
\end{tabular}




\subsection{Modeling random access for $\mathrm{H} 2 \mathrm{H}$ and PM2M traffic}

We now present the analytical model of random access, beginning with $\mathrm{H} 2 \mathrm{H}$ terminals. Although the population of $\mathrm{H} 2 \mathrm{H}$ terminals in a single LTE/LTE-A cell is large, they attempt random access occasionally and infrequently; thus, we may safely assume that $\mathrm{H} 2 \mathrm{H}$ requests arrive according to a Poisson distribution. The mean arrival rate $\lambda_{w}$ of $\mathrm{H} 2 \mathrm{H}$ traffic can be calculated for a single PRACH resource as, $\lambda_{W H}=\left(\lambda_{w} T_{f}\right) / c_{f}$ where, $T_{f}$ is the LTE superframe time and $c_{f}$ determines the number of PRACH resources per LTE frame. LTE PRACH is overloaded by preamble collision and external interference caused by the surrounding networks like VANET. To detect a signal successfully at eNodeB of LTE the error rate should be low and signal to interference noise ratio (SINR) should not be under the certain threshold value.

In the first and third step of 4 way handshaking mechanism, LTE results in excessive uplink transmission and violets the SINR threshold and cause overload on PRACH. H2H networks SINR (in decibels) is the ratio of preamble sequence energy over noise power density for $n$ number of $\mathrm{H} 2 \mathrm{H}$ traffic including initial and handoff requests and all the transmissions and receiving are done with same power level.

$$
\begin{aligned}
E_{\text {seq }} / N_{0} & =\frac{\frac{P_{s e q} I_{1}}{R}}{\frac{(n-1) I_{1}+N_{M} I_{1}+\eta_{p, 1}+\eta_{0} W}{W}} \\
& =P_{\text {seq }} \frac{W / R}{n-1+N_{M}+\eta_{p, 1} / I_{1}+\eta_{0} W / I_{1}}
\end{aligned}
$$

where,

$P_{\text {seq }}=$ preamble sequence length in bits 
$\mathrm{W}=\mathrm{LTE}$ bandwidth

$\mathrm{R}=\mathrm{LTE}$ PRACH preamble data rate

$I_{1}=$ Received signal power

$\eta_{p, 1}=$ outer cell interference power

$\eta_{0}=$ spectral density of white noise

$N_{M}=\mathrm{PM} 2 \mathrm{M}$ preamble code which are always active

Detection of the successful preamble over the preamble sequences depends on the ratio of $E_{\text {seq }} / N_{0}$. SINR of preamble detection is $10 \log \frac{E_{\text {seq }}}{N_{0}}$ for PM2M traffic. Threshold value of $18 \mathrm{~dB}$ (or $-11.23 \mathrm{~dB}$ ) guarantees that probability of preamble missed detection is smaller than $10^{-2}$ and probability of false alarm is less than $10^{-3}$ for eNodeB. The ratio of the outer cell interference and received signal power $\eta_{p, 1} / I_{1}$ of $\mathrm{PRACH}$ is a Gaussian distribution with mean and variance denoted by $k_{m, 1}$ and $k_{v, 1}$ [61]. In case of threshold exceed the overload condition occurs and preamble can not be detected successfully. The probability of PRACH overload in the first handshake step is,

$$
O_{-} P R A C H_{n}=P_{r}\left(n+\frac{\eta_{p, 1}}{I_{1}}>\frac{W}{R T_{1}}-N_{M}-\frac{W \eta_{0}}{I_{1}}\right)
$$

The PRACH overload for third handshake step for $j$ number of $\mathrm{H} 2 \mathrm{H}$ traffic is,

$$
Q_{-} P R A C H_{j}=P_{r}\left(j+\frac{\eta_{p, 3}}{I_{3}}>\frac{W_{3}}{R_{3} T_{3}}-\frac{W_{3} \eta_{0}}{I_{3}}\right)
$$

The third step also carries important information and we assume that the spectral efficiency of uplink transmission is as same as first step, $\frac{W_{3}}{R_{3}}=\frac{W}{R}$. This steps threshold value is $-5 \mathrm{~dB}$ because it is observed over a single bit. The ratio of the outer cell interference and 
received signal power $\frac{\eta_{p, 3}}{I_{3}}$ of PRACH is a Gaussian distribution with mean and variance denoted by $k_{m, 3}$ and $k_{v, 3}[61]$.

\subsubsection{H2H PRACH overload during preamble collision}

LTE PRACH is overloaded by preamble collision. To calculate the PRACH overload we need to know the total Poisson arrival rate of per PRACH for all the traffic including new, returning and handover calls, $\lambda_{\text {total }}=\lambda+\lambda_{p c 1}+\lambda_{p c 3}+\lambda_{h}$. Now we need to find the probability of $n$ arrivals of neither new or handover $\mathrm{H} 2 \mathrm{H}$ calls is,

$$
P_{n}=\frac{\left(\lambda_{\text {total }}\right)^{n}}{n !} e^{-\lambda_{\text {total }}}
$$

For $n \mathrm{H} 2 \mathrm{H}$ access attempts in PRACH resources, from overload equation of 4.2 we obtain the overload probability as,

$$
O_{-} P R A C H_{n}=\operatorname{erfc}\left(\frac{\frac{W}{R} \frac{1}{T_{1}}-\frac{W \eta_{0}}{I_{1}}-n+1-N_{M}-k_{m, 1}}{\sqrt{k_{v, 1}}}\right)
$$

where, erfc is a complementary error function,

$$
\operatorname{erfc}(x)=\frac{2}{\sqrt{(\Pi)}} \int_{x}^{\infty} e^{-\frac{t^{2}}{2}} d t
$$

Thus the total overload probability for first handshake step due to collision and interference is obtain by averaging the $\mathrm{H} 2 \mathrm{H}$ load:

$$
P_{O, 1}=\sum_{n=2}^{\infty} P_{n} O_{-} P R A C H_{n}
$$


by using euation 4.4 and 4.5 ,

$$
=\sum_{n=2}^{n_{\max }} \frac{\left(\lambda_{\text {total }}\right)^{n}}{n !} e^{-\lambda_{\text {total }}} O_{-} P R A C H_{n}
$$

For the third handshake step collision probability that $\mathrm{H} 2 \mathrm{H}$ terminals collide when $j>1$ is,

$$
P_{3}(j)=e^{-\lambda_{i}, L_{3}} \frac{\lambda_{i, L_{3}}^{j}}{j !}
$$

where,

$$
\lambda_{i, L_{3}}=\text { arrival rate of third step L2/L3 messages }
$$

Now to find the total overload of PRACH for third handshake step the overload probability of equation 4.3 can be written with complementary error function as equation 4.6 with different values of bandwidth, spectral efficiency and SINR.

$$
Q_{-} P R A C H_{j}=\operatorname{erfc}\left(\frac{\frac{W_{3}}{R_{3}} \frac{1}{T_{3}}-\frac{W_{3} \eta_{0}}{I_{3}}-j-k_{m, 3}}{\sqrt{k_{v, 3}}}\right)
$$

Thus the overall PRACH overload probability for third handshake step with preamble collision and inter cell interference is,

$$
P_{Q}=\frac{\sum_{j=2}^{j} P_{3}(j) Q_{-} P R A C H_{j}}{\sum_{j=2}^{j} P_{3}(j)}
$$

\subsubsection{PM2M overload calculation}

Bit error rate (BER) of overlay PM2M depends on the $\mathrm{H} 2 \mathrm{H}$ traffic of the PRACH. Through the BER, PM2M traffic knows the presence of $\mathrm{H} 2 \mathrm{H}$ traffic. To estimate the BER 
of PM2M traffic we need to find the overload probability of PM2M based on SINR requirements for PRACH. SINR of PM2M traffic is,

$$
\begin{aligned}
E_{b} / N_{0} & =\frac{\frac{N_{b} I_{1}}{R}}{\frac{(n) I_{1}+\left(N_{M}-1\right) I_{1}+\eta_{p, 1}+\eta_{0} W}{W}} \\
& =N_{b} \frac{W / R}{n+N_{M}-1+\eta_{p, 1} / I_{1}+\eta_{0} W / I_{1}}
\end{aligned}
$$

where,

$N_{b}=$ preamble elements

$\mathrm{W}=\mathrm{LTE}$ bandwidth

$\mathrm{R}=\mathrm{LTE}$ PRACH preamble data rate

$I_{1}=$ Received signal power

$\eta_{p, 1}=$ outer cell interference power

$\eta_{0}=$ spectral density of white noise

$N_{M}=$ PM2M preamble code which are always active

The overload probability of PM2M data on each preamble is depends on the $\mathrm{H} 2 \mathrm{H}$ traffic and inter cell interference. It can be derived as a same way which is analogous to equations 4.5 and 4.2

$$
\begin{gathered}
M_{-} \text {oload }_{n}=P_{r}\left(n+\frac{\eta_{p, 1}}{I_{1}}>\frac{W N_{b}}{R T_{M}}-N_{M}-\frac{W \eta_{0}}{I_{1}}\right) \\
=\operatorname{erfc}\left(\frac{\frac{W N_{b}}{R} \frac{1}{T_{M}}-\frac{W \eta_{0}}{I_{1}}-n-N_{M}+1-k_{m, 1}}{\sqrt{k_{v, 1}}}\right)
\end{gathered}
$$

The total PM2M overload probability can be obtain as, 


$$
P_{M \_o l o a d}=\sum_{n=0}^{\infty} P_{n} M_{-} \text {oload }{ }_{n}
$$

by using equation 4.13 and 4.4 ,

$$
=\sum_{n=2}^{n_{\max }} \frac{\left(\lambda_{\text {total }}\right)^{n}}{n !} e^{-\lambda_{\text {total }}} M_{\text {_oload }}
$$

using the PM2M overload probability we could approximate the BER as half of the overload.

$$
B E R_{P M 2 M}=0.5 P_{M \_o l o a d}
$$

\subsubsection{Performance of $\mathrm{H} 2 \mathrm{H}$ traffic in the presence of overlay Network}

We consider LTE cells with mix of H2H and M2M traffic, using the PRACH and PM2M overlay parameters given in Table 4.2. We evaluate the performance of $\mathrm{H} 2 \mathrm{H}$ traffic in the presence of PM2M traffic for simultaneous access on RACH. We assume that number of codes per cell is $\mathrm{N}=64$, number of preambles reserved for handoff is $N_{h}=10$ and number of preambles reserved for physical layer of PM2M overlay is $N_{M}=8$. This leaves $N_{i}=$ 46 preambles for $\mathrm{H} 2 \mathrm{H}$ access. One data bit in overlay PM2M network is spread over $N_{b}$ $=16$ preamble elements. In the experiment we consider $c_{f}=5, \mathrm{PF}=2$ which means that 5 independent PRACH resources are available in one LTE frame. Each PRACH resource has preamble duration of $800 \mu \mathrm{s}$. We pointed out that, $\mathrm{PF}=2$ provides large guard times, can handle higher mobility, provides larger cell and can handle handover calls greatly. We consider scenario as shown in figure 4.4 and evaluate the performance of following experiment: 
$P F=2, c_{f}=5$ with $\mathrm{H} 2 \mathrm{H}$ request arrival rate is varying between 20 and 220 requests per second.

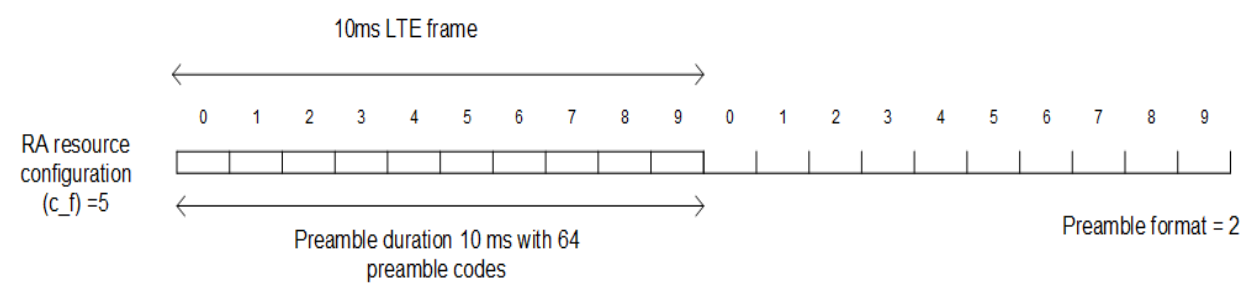

Figure 4.4: Random access resource allocation for $\mathrm{PF}=2$ with $c_{f}=5$

In every cell we have $\mathrm{H} 2 \mathrm{H}$ and $\mathrm{PM} 2 \mathrm{M}$ traffic so we always want to have overlay which is always sufficient for some traffic. We are taking the minimum $N_{M}$ codes so that it could handle the PM2M traffic. We evaluate the performance by varying the $\mathrm{H} 2 \mathrm{H}$ traffic between 20 to 220 calls/s to find the capacity limit of the network under the presence of PM2M traffic when $N_{M}=8$ preambles are permanently active as physical layer of PM2M overlay.

Fig. 4.5a shows $\mathrm{H} 2 \mathrm{H}$ probability of success, which remains within $99 \%$ up to $220 \mathrm{H} 2 \mathrm{H}$ calls/s. The collision probability shown in Fig. $4.5 \mathrm{~b}$ is only 0.0045 which validates the probability of success. The presence of overlay network is not overloading the cell with respect to $\mathrm{H} 2 \mathrm{H}$ traffic as shown in Fig. 4.5c where we observe that, for $\mathrm{H} 2 \mathrm{H}$ arrival rate up to 220 calls/s, the overload probability for $\mathrm{H} 2 \mathrm{H}$ traffic is only 0.00065 which is very low indeed. The mean access delay (Fig. 4.5d) is essentially flat, with only a slight increase from $14.500 \mathrm{~ms}$ to $14.556 \mathrm{~ms}$ in the observed range. Note that these performance limits would apply to M2M devices in case they use regular LTE access on PRACH: in other words, random access procedure on PRACH is able to accommodate only about 220 calls per second, be they posted by $\mathrm{H} 2 \mathrm{H}$ or M2M users. 

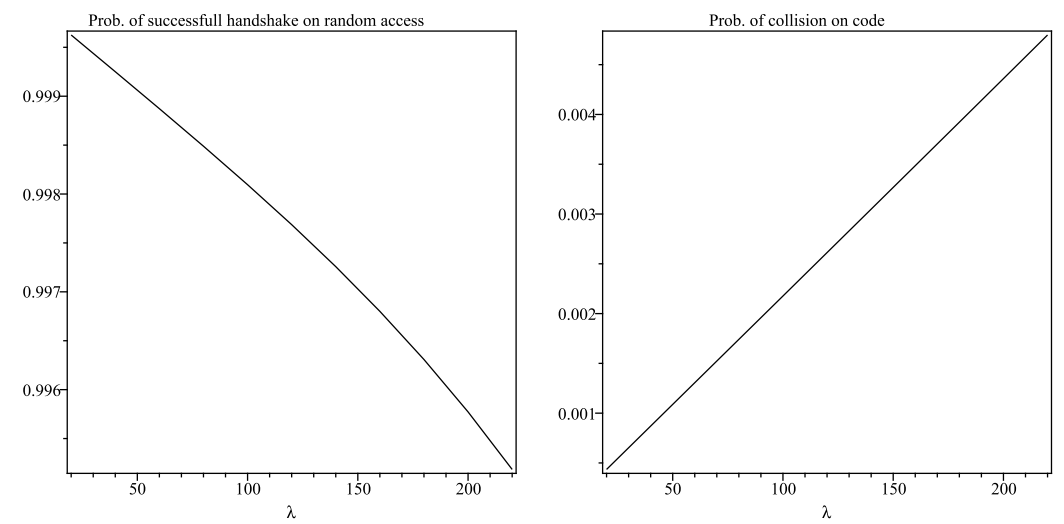

(a) Probability of successful handshake(b) Probability of code collision on on PRACH

PRACH

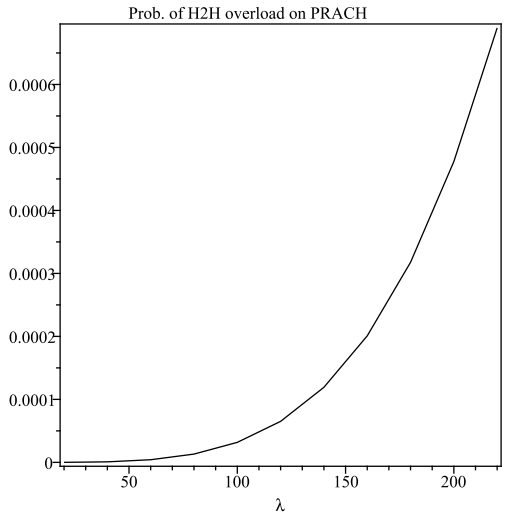

(c) Probability of PRACH overload

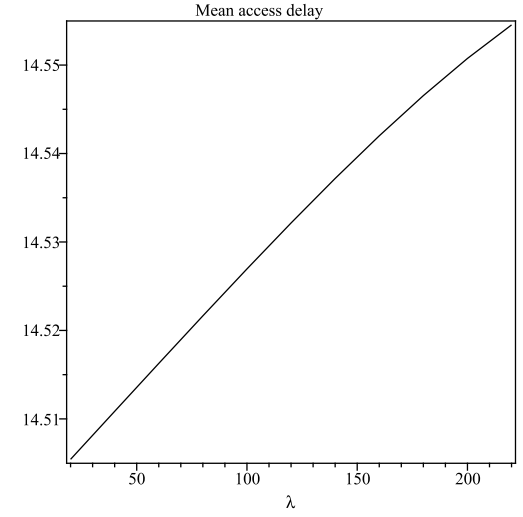

(d) $\mathrm{H} 2 \mathrm{H}$ Mean Access Delay in ms

Figure 4.5: Performance of $\mathrm{H} 2 \mathrm{H}$ traffic for $P F=2 c_{f}=5$

\subsection{Modeling PM2M overlay network backoff procedure with backoff error}

In this section, we model the analytical model for CSMA/CA backoff procedure with backoff error. We consider a single hop PM2M overlay network with $\mathrm{H} 2 \mathrm{H}$ terminals in a single LTE/LTE-A cell. We assume that only one data frame arrives at the queue during a single CSMA slot. Random access channel will have difficulty in handling a large number 
of short data transmissions from PM2M, due to a large number of preamble collisions in first handshake step and overload conditions where SINR at eNodeB is violated. RACH resources are shared by all user equipment, which results in congestion in the network and shortage of resources. The number of preambles is limited and hence, there is a possibility that more than one mobile terminal chooses the same preamble that results in preamble collision. The use of four-way handshaking procedure of LTE for massive network access leads to network overload. To detect a signal successfully at eNodeB of LTE the error rate should be low and signal to interference noise ratio (SINR) should not be under the certain threshold value. Detection of the successful preamble over the preamble sequences depends on the ratio of $E_{\text {seq }} / N_{0}$. SINR of preamble detection is $10 \log \frac{E_{\text {seq }}}{N_{0}}$ for PM2M traffic. The threshold value of $-11.23 d B$ guarantees that probability of preamble missed detection is smaller than $10^{-2}$ and probability of false alarm is less than $10^{-3}$ for eNodeB. The ratio of the outer cell interference and received signal power $\eta_{p, 1} / I_{1}$ of PRACH is a Gaussian distribution with mean and variance denoted by $k_{m, 1}$ and $k_{v, 1}$ [61]. In case of threshold exceed, the overload condition occurs and preamble cannot be detected successfully. The probability of PRACH overload is described in detail in Section 4.4.

We develop an analytical model which consider backoff error during CSMA/CA mechanism for non-saturation condition where the queue will not always have packet to transfer and each of the nodes has to calculate the idle period when queue is empty. The model consists of two 3 dimensional discrete time Markov chain to compute the medium access probability for all UPs, one for each UPs. We calculate all backoff phase during CSMA/CA mechanism by extending the 3 dimensional to 4 dimensional DTMCs to calculate the backoff duration of all UPs. In [72] the authors describe a non saturation model for 
interconnecting IEEE 802.15.6 with WLAN and they also consider RTS/CTS mechanism. The authors did not consider the backoff error which could present during the CSMA/CA mechanism. The physical layer of our proposed architecture is different than [72] as our physical layer is implemented with dedicated preambles and the use of RTS/CTS will create an extra overload on the PRACH of LTE.

We consider 2 user priorities, $U P_{k}, \mathrm{k}=0,1$, where index 0 means lower and index 1 means higher priority traffic classes. All the time scales are considerate in slots and they are modified according to LTE time scale. The backoff value of a $U P_{k}$ node is calculated uniformly over the interval $\left[1, C W_{k}\right] . C W_{k}$ has minimum value of $C W_{k, \min }=W_{k, i}$ where $i=0, i$ can be up to transmission retry limits $\mathrm{R}$ and maximum contention window value $C W_{k, \max }=W_{k, \max _{k}}$, the value is increased up to maximum number of retries $\mathrm{R}$, where $R=0 \ldots 7$. The user priorities are differentiate according to the value of $C W$ values (Table 4.1). We assume that if the data frame exceeds the number of retry limits $\mathrm{R}$ then the packet will be dropped. Contention window values for a user priorities $U P_{k}$ node for ith backoff phase is calculated as follows:

- Initially, $W_{k, i}=W_{k, \min }=C W_{k, \min }$, where $i=0$.

- Contention window value doubles when, $W_{k, i}=\min \left\{2 W_{k, i-1}, C W_{k, \max }\right\}$ for , $2<=i<=R$, if $i$ is an even number

- Contention window value increase uniformly when, $W_{k, i}=W_{k, i-1}$ for $1<=i<=$ $R$, if $i$ is an odd number.

Bit error rate (BER) coming from the $\mathrm{H} 2 \mathrm{H}$ (section 4.4) has great impact on the PM2M overlay network. PM2M node will know the presence of $\mathrm{H} 2 \mathrm{H}$ traffic through the BER. We 
calculate the probability of neither the data nor the acknowledgement (ack) is corrupted by noise as,

$$
\sigma=\left(1-B E R_{P M 2 M}\right)^{d t_{s}+a k_{s}}
$$

Where

$$
\begin{gathered}
B E R_{P M 2 M}=\text { BER coming from } \mathrm{H} 2 \mathrm{H} . \\
d t_{s}, a k_{s}=\text { data and acknowledgement size in slots. }
\end{gathered}
$$

In IEEE 802.15.6 during the medium access mechanism through CSMA/CA a node sense the medium while decrementing the backoff counter. If the medium is sensed to be busy it freezes the backoff counter and when medium sensed idle again it decrements the backoff counter. $\mathrm{H} 2 \mathrm{H}$ traffic is also transmitting in parallel with the PM2M traffic then there is a possibility that we might not listen the medium right over the backoff period. The PM2M overlay could hear the noise because of the $\mathrm{H} 2 \mathrm{H}$ traffic. Maybe medium as idle but because of the activity we may sense it as busy, also when medium is busy we may hear it as idle. Bit error rate (BER) coming from the $\mathrm{H} 2 \mathrm{H}$ layer has great impact on the PM2M layer. BER is the probability of hearing one bit wrong. During accessing the medium nodes sense the status of the medium in backoff period, where 1 backoff period is equivalent to 20 bit times. We have 20 bits and listening of each bit can be wrong from the probability of BER. If all of them or more than half of the bits are wrong we have the wrong listening decision which actually depends on the hardware of the networks. In our case, we define the probability of backoff error as the probability of listening 11 bits are wrong among 20 bits and less than 11 bits are correct. We calculated the probability of backoff error (PBerr) which is a binomial distribution: 


$$
P B_{\text {err }}=\sum_{B=11}^{20}\left(\begin{array}{c}
20 \\
B
\end{array}\right) B E R_{P M 2 M}^{B}\left(1-B E R_{P M 2 M}\right)^{20-B}
$$

where

$$
\begin{gathered}
B E R_{P M 2 M}=\text { Bit error rate coming from } \mathrm{H} 2 \mathrm{H} \\
B=\text { Physical bit }
\end{gathered}
$$

We develop a 3 dimensional Discrete Time Markov chain (DTMCs) to model the backoff procedure of the CSMA/CA mechanism with backoff error only for two traffic classes and random access phase (RAP) is depicted in Fig. 4.6. To calculate the average backoff time, we calculate all possible backoff phase during CSMA/CA mechanism and extend 3 dimensional DTMCs to 4 dimensional DTMCs for all UPs as shown in Fig. 4.7. We evaluate the important network performance descriptors with the presence of backoff error: average backoff time, mean number of backoff attempts, medium access probability and normalized throughput.

Medium access probability $\tau_{k}$ of a $U P_{k}$ node, where $k=0,1$ during random access phase of RAP is calculated by solving 2 dependent discreet time Markov chain (DTMC ) described in [72]. It is an interactive approach and CSMA slots during which backoff countdown is paused, is not considerate, which improves the accuracy of calculated access probabilities. The probability is calculated only when the medium is idle and nodes are competing to get access. We rewrite all the equations of [72] to consider the Markov chain with backoff error and to calculate the number of backoff attempts by each nodes and change the parameter values according to our proposed solutions. During the busy period the access probability is counted as zero. With the presence of the backoff error, while we 


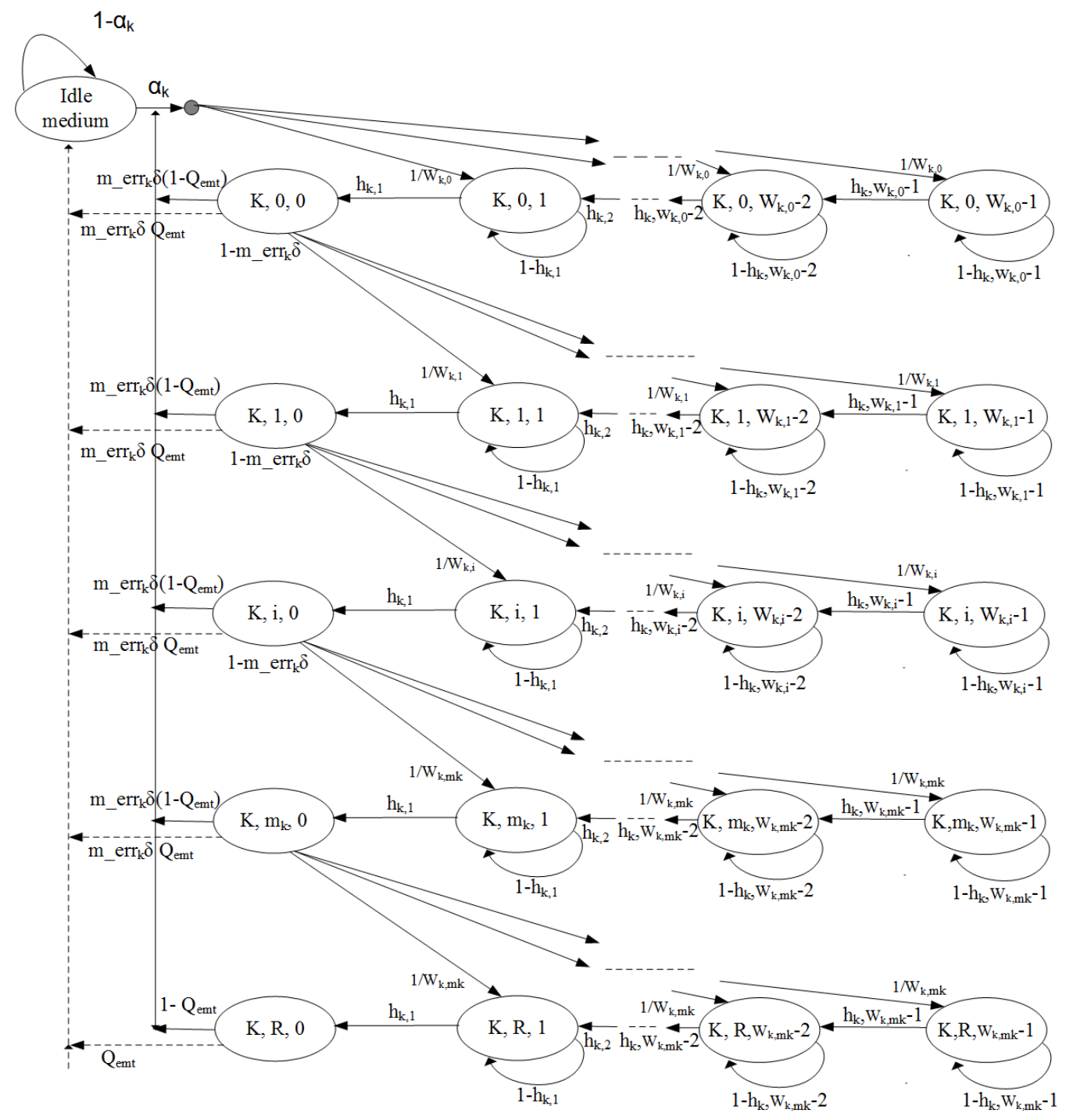

Figure 4.6: Markov chain for $U P_{k}$, modified from [72]

are sensing the medium as idle there is a probability that medium is not idle. When one node is transmitting the other nodes have to wait for long time because of backoff error in Markov chain. The wrong idle medium probability in CSMA slots where all user priority nodes can access is: 


$$
m_{-} i d l e_{\text {Aerr }}=\sum_{i=0}^{7} P B_{\text {err }}\left(1-\tau_{i}\right)^{n_{i}}
$$

where

$$
n=\text { number of nodes in each traffic class }
$$

Due to the presence of backoff error we could detect wrong idle medium probability, when we say medium is idle but there is a probability that medium is busy. The wrong detection of medium status will have affect on the entire backoff process. The busy medium probability is

$$
m \_b u s y=1-m_{-} i d l e_{A e r r}
$$

Then we evaluate the probability of medium idle during backoff countdown and that means other nodes do not access the medium during current access phase RAP is,

$$
m_{-} e r r_{k}=\frac{m_{\_} i d l e_{\text {Aerr }}}{1-\tau_{k}}
$$

where

$$
k=0 \ldots 1
$$

When a node is decrementing the backoff count there is a possibility that there is not enough time in the superframe (RAP) to complete the countdown and complete the transmission. We have to introduce $S_{-} t i m e_{k}$, which is the probability that there is not enough time to complete the current transmissions. Initially we set the initial RAP access period (superframe values in backoff periods) values and then it is calculated in each iteration in order to approximate backoff process which depends on idle medium probability, medium 
access probability, frame success $T_{\text {succ }}$ and collision time $T_{\text {coll }}$, nodes backoff stage and uncorrupted nodes. The values of probability of $S_{-} t i m e_{k}$ is,

$$
S_{-} \text {time }_{k}=\frac{1}{X_{R A P}-T_{\text {succ }}-C_{k}}
$$

where

$$
\begin{gathered}
T_{s u c c}=\left(\text { data }_{s}+a c k_{s}+\text { sifs }\right), \text { successful transmission time in slots } \\
C_{k}=\frac{C W_{k, \min }+C W k, \max }{4}, \text { approximate mean backoff counter value } \\
T_{\text {coll }}=\left(\text { data }_{s}+a c k_{s}+\text { sifs }\right) \text { unsuccessful transmission time }
\end{gathered}
$$

Now we have to calculate the probability that backoff counter value of a node decrements when the value is equal to $\mathrm{j}$, where, $j=1, \ldots, W_{k, m_{k}}$

$$
h_{k, j}=m_{-} \operatorname{err}_{k}\left(1-S_{-} \operatorname{time}_{k} \frac{1-m_{-} e r r_{k}^{j}}{1-m_{-} e r r_{k}}\right)
$$

The state probabilities $b_{k, i, j}$ of Markov chain as shown in figure 4.6 is a random process where, $k=0 \ldots 1, i=0 \ldots R$ and $j=0 \ldots W_{k, i}$. The medium access probability is calculated in the state which is equal to,

$$
\tau_{k}=\sum_{0}^{R} b_{k, i, o}
$$

We have to calculate the zero-th backoff phase which depends on the probability of CSMA slot is in idle state, probability that queue is empty due to successful transmission or dropped because of exceeded retry limit. In our model we did not formulate the queue model we consider probability of queue empty $Q_{e m t}$ from [72]. The probability of being in idle state may refers to wrong medium status as presence of backoff error, is calculated as: 


$$
S_{i d l e, k}=P B_{\text {err }} \frac{\tau_{k} m_{-} e r r_{k} \sigma_{k} Q_{e m p t}}{\alpha_{k}\left(1-\left(1-m_{-} e r r_{k} \sigma_{k}\right)^{R+1}\right)}
$$

where

$\alpha=$ The probability of data frame arrives during the interval of between two successive Markov points.

Thus the equation of zero-th backoff phase is,

$$
Z_{b o f f, k}=\frac{\tau_{k} m_{-} e r r_{k} \sigma_{k}\left(1-Q_{e m t}\right)+S_{i d l e, k} \alpha}{1-\left(1-m_{-} e r r_{k} \sigma_{k}\right)^{R+1}\left(1-Q_{e m t}\right)}
$$

By the normalized condition of DTMCs the summation of all the probability is equal to 1 and by solving all the equations we calculate medium access probability with backoff error, $\tau_{k}$ of a $U P_{k}$ node, where $k=0 \ldots 1$ during random access phase of RAP.

$$
1=S_{i d l e, k}+Z_{b o f f} \sum_{i=0}^{R}\left(1-m_{\_} e r r_{k}\right)^{i}\left(1+\sum_{j=1}^{W_{k, i}} \frac{W_{k, i}-j+1}{W_{k, i} m_{\_} e r r_{k} \sigma_{k}}\right)
$$

All nodes has to calculate the probability of empty queue after successful transmission or dropped due to exceeded retry limit. We have to calculate the backoff duration. During CAMA/CA mechanism when a node tries to get access to medium has to backoff and lock the backoff counter for the following situations

- Successful transmission by another node i.e. channel is busy

- Unsuccessful transmission by another node

- Not enough time to complete the frame transmission in current superframe time. 
The node unlocks the backoff counter again when the following conditions occur:

- The channel is idle for SIFs amount of time within the superframe time

- Current superframe has enough time to complete the frame transaction.

All of the above conditions works fine when there are no backoff error presents in the channel. In presence of backoff error the node could listen the medium as idle and unlock the backoff counter when actually it is busy. These situation could congested the network and overload the LTE PRACH. We added the backoff error in the Markov chain backoff procedure when sensing the medium for idle or busy as shown in Fig. 4.7 and calculated the PGFs of times for the following moments. The upper part of figure 4.7 probability distribution states, $b_{k, i, j}$ shows details in lower part.

- $\operatorname{Ebfsucc}_{k, j}(z)$, the moments between the locking and unlocking the backoff counter due to successful transmission by another node

- $\operatorname{Ebfcoll}_{k, j}(z)$, the moments for unsuccessful transmission

- $\operatorname{Ebfploc}_{k, j}(z)$, the moments between locking and unlocking when there is not enough time for completing a frame transaction.

All of the above conditions are considerate for only RAP access phase where, $k=0 . .1$ UPs and $j=1 . . W_{k, m_{k}}$, specifies backoff counter value for locked condition. We calculate the PGFs of all the above moments and total number of backoff attempts before a node can successfully access the medium. The PGFs are computed as follows, 


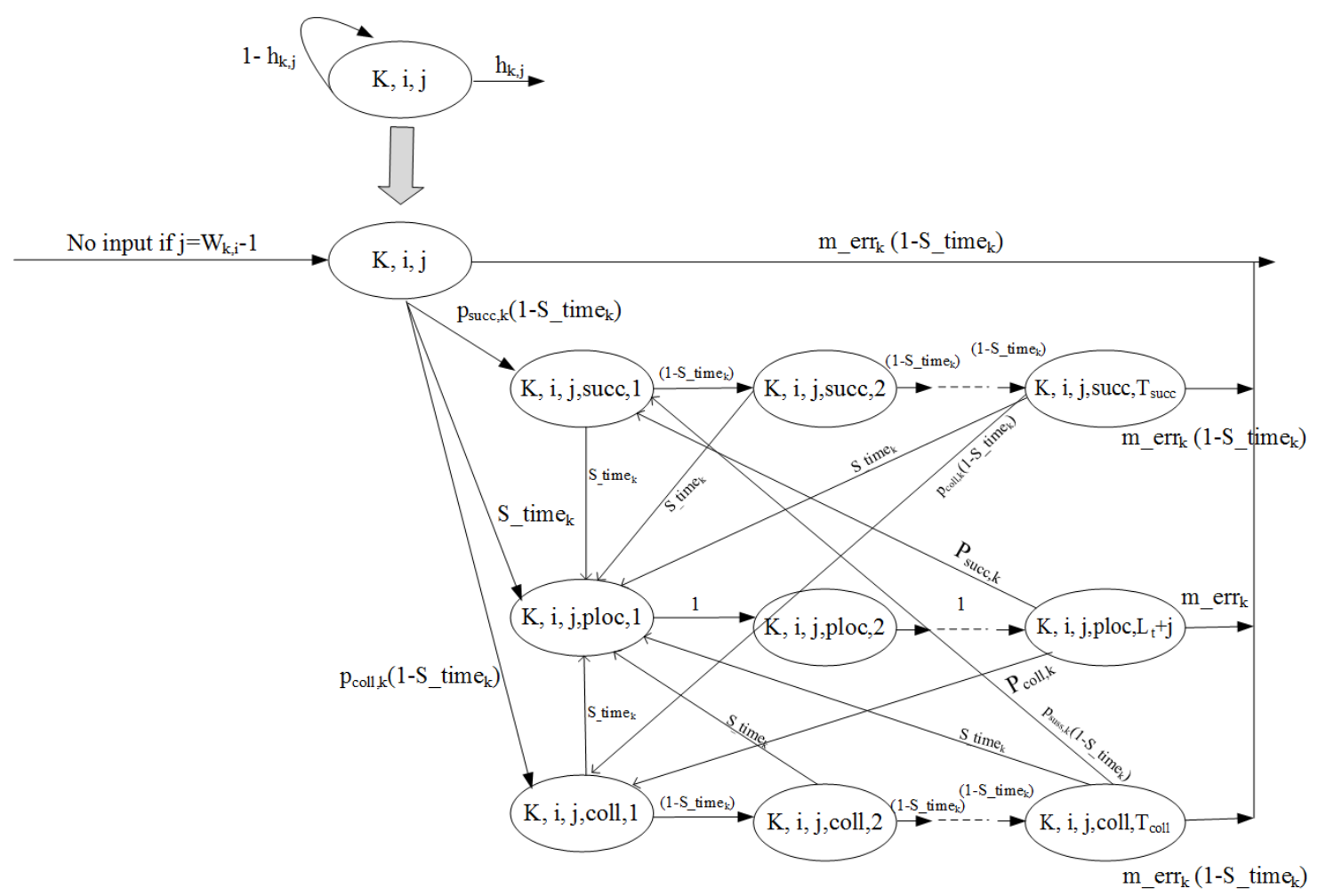

Figure 4.7: Extended Markov chain for $U P_{k}$ modified from [72]

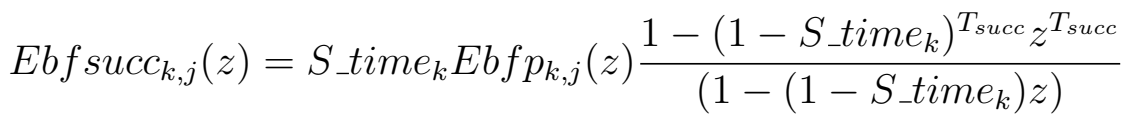

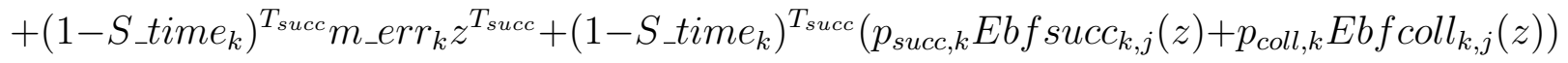




$$
\begin{aligned}
& \left.\operatorname{Ebfcoll}_{k, j}(z)=S_{\_} \text {time }_{k} \operatorname{Ebfp}_{k, j}(z)\left(1-\left(1-S_{-} \text {time }_{k}\right)^{T_{\text {coll }}} z^{T_{\text {coll }}}\right) /\left(1-\left(1-S_{-} \text {time }_{k}\right) z\right)\right) \\
& +\left(1-S \_t i m e_{k}\right)^{T_{\text {coll }}} m_{-} \text {err }_{k} z^{T_{\text {coll }}}+\left(1-S \_t i m e_{k}\right)^{T_{\text {coll }}}\left(p_{\text {succ }, k} E_{b f f u c c_{k, j}}(z)\right. \\
& \left.+p_{\text {coll }, k} \operatorname{Ebf} \operatorname{coll}_{k, j}(z)\right)
\end{aligned}
$$

$$
\operatorname{Ebfploc}_{k, j}(z)=z^{L_{t, k}+j}\left(m_{-} \operatorname{err}_{k} z+\left(p_{\text {succ }, k} \operatorname{Ebfsucc}_{k, j}(z)+p_{\text {coll }, k} \operatorname{Ebfcoll}_{k, j}(z)\right)\right)
$$

Here

$S_{-}$time $_{k}=$ probability that there is not enough time for completing a frame transaction in current RAP.

$T_{\text {succ }}=($ data + ack +3 sifs $) \mathrm{s}$, successful transmission time in slots.

$T_{\text {coll }}=$ unsuccessful transmission time in slots

$P_{s u c c, k}=$ probability to lock the backoff counter due to successful transmission by others $P_{c o l l, k}=$ probability to lock the backoff counter due to unsuccessful transmission by others $L_{t}=\left(X_{R A P}+d a t a+a c k+3 s i f s\right)$ s number of CSMA slots when backoff counter must be locked because not enough time for completing transaction.

All of the above equations are solved to compute the values of unknown variables and model the backoff counter locked times based on the transition probabilities of Markov chain extended model with backoff error shown in 4.7.

Now we can calculate the PGF of time to decrement the backoff counter by one which is time interval of the moments when backoff counter reaches $j$ to the moment when it 
becomes $j-1$. The PGFs of this moment is,

$$
\begin{aligned}
& B f C_{k, j}(z)=S_{\_} \text {time }_{k} E b f p l o c_{k, j}(z)+\left(1-S_{\_} \text {time }_{k}\right) \\
& \left(m_{-} \operatorname{err}_{k} z+P_{\text {succ, }, k} E b f \operatorname{succ}_{k, j}(z)+P_{\text {coll }, k} E b f \operatorname{coll}_{k, j}(z)\right)
\end{aligned}
$$

Now due to probabilistic nature of Markov chain it is uncertain that after backoff stage $b_{k, i, j, 1}$ next stage will be $b_{k, i, j-1,2}$ or $b_{k, i, j-1,3}$. We define the probability as $P S t_{k}$ and calculated the probability by using the transition probability in the extended Markov chain 4.7 where, $i=0 . . R, j=0 . . W_{k, i}$ :

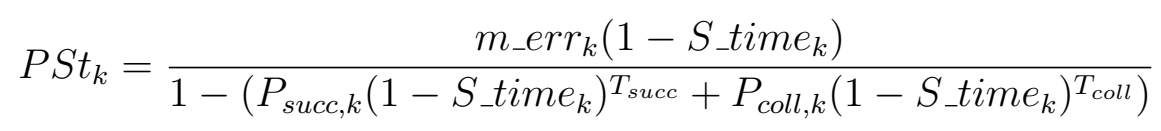

Now the PGF of backoff time for $i-t h$ backoff phase is calculated as the moments when backoff value is randomly selected until countdown reaches 0 .

$$
B f I_{k, i, 1}(z)=B f C_{k, 1}(z) B f I_{k, i, j}(z)=B f C_{k, j}(z)\left(P S t_{k} B f I_{k, i, j-1}(z)+\left(1-P S t_{k}\right)\right)
$$

By developing the above PGF function we can compute the PGF of backoff time and number of backoff attempts.

We calculate the backoff time $\left(B f T_{k}\right)$ which is defined as the total time used during the 
backoff procedure until successful access to the medium or dropped due maximum number of retry limit $\mathrm{R}$.

$$
B f T_{k}(z)=R A P_{s l o t} B f I_{k, i}(z)
$$

Here

$R A P_{\text {slot }}=$ Mean number of slots in our superframe.

Mean number of backoff time is,

$$
\text { Btime }_{k}=\left.\frac{\partial}{\partial z} B f T_{k}(z)\right|_{z=1 \omega}
$$

We study the network performance descriptor mean number of backoff attempt $\left(B_{-} N_{a m p, k}\right)$ by each node before successfully access the medium to transmit the data packet with backoff error,

$$
b_{\text {attmp }, k}=m_{-} e r r_{k} \sigma[k], k=0 \ldots 1
$$

which is a truncated geometric distribution.

$$
B_{\text {attmp }}(X=R)=\sum_{R=1}^{7}\left(b_{\text {attmp }, k}\left(1-b_{\text {attmp }, k}\right)^{R-1}\right)
$$

Here, $\mathrm{R}=$ maximum number of attempts to transmit the packet or retry limits. We calculate the PGF of every backoff attempt and then calculate the total backoff attempt before accessing the medium by each node $U P_{k}$ where $k=0$ and 1 . 


$$
B_{\text {att }, k}(z)=\left(b_{\text {attmp }, z}\right)\left(1-\left(\left(1-b_{\text {attmp }, k} z\right)\right)^{R+1}\right) /\left(1-\left(1-\left(b_{\text {attmp }, k} z\right)\right)\right)
$$

The mean number of backoff attempts before successfully access the medium and exceed the retry limit $\mathrm{R}$ is

$$
B_{-} N_{a t t m p, k}=\left.\frac{\partial}{\partial z} B_{a t t, k}(z)\right|_{z=1}
$$

The throughput is calculated as time to transmit the pack including backoff time, total transmission time and packet successful transmission time on the medium. It is the ratio of actual transmission time over the total time needed to prepare the transmission. We compute the normalized throughput of a node of a $U P_{k}$, where $\mathrm{k}=0 . .1$, with respect to the mean backoff time as a denominator as follows:

$$
\operatorname{Thro}_{k}=\frac{\sigma d t_{s} P_{\text {succ }} \omega}{\text { Btime }_{k}}
$$

Here

$\sigma=$ The probability of neither the data nor the acknowledgement (ack) is corrupted by noise. $d t_{s}=$ payload size of data in slots without headers.

$P_{\text {succ }}=$ Probability of data successful transmission on the medium which is calculated as,

$=\sigma\left(1-\left(1-m_{-} e r r_{k} \sigma\right)^{R}\right)$

$m_{-} e r r_{k}=$ Probability of medium ideal (other nodes do not access the medium).

$\omega=$ Length of one CSMA/CA slot.

Btime $_{k}=$ Mean backoff time. 


\subsection{Performance evaluation of PM2M overlay network}

We evaluate the performance of PM2M overlay network for two traffic classes and only for RAP contention based access phase. The results are produced by solving the numerical calculations of the analytical models in Maple [56]. We evaluate the performance with and without backoff error and compare the performance for both traffic classes while $\mathrm{H} 2 \mathrm{H}$ traffic is fixed for 100 calls/sec. We investigate the capacity and seamless communications of PM2M to access PRACH with parallel to the $\mathrm{H} 2 \mathrm{H}$ traffic. We only consider IEEE 802.15.6 Random Access Phase (RAP) and non saturation condition which means that the buffer will not always have data frame to transmit. In this work, we assume that data packet size is 30 bytes including MAC headers. Data packet should contain cell and node IDs. Among 30 bytes packet size, 10 bytes are reserved for MAC header and other 20 bytes are left for actual MAC data. We evaluate the important network performance descriptors: average backoff time, the mean number of backoff attempts, medium access probability and normalized throughput. Table 4.2 shows the implementation parameters with values. In order to validate the correctness of our proposed method with respect of probability of success and access delay, we compare our approach with a reference approach [87] with the presence of fixed $\mathrm{H} 2 \mathrm{H}$ traffic intensity. We have conducted following three experiments:

- Performance evaluation of PM2M network in the presence of $\mathrm{H} 2 \mathrm{H}$.

- Performance comparison of PM2M overlay network with reference approach in the presence of $\mathrm{H} 2 \mathrm{H}$.

- Performance evaluation of PM2M network in the presence of $\mathrm{H} 2 \mathrm{H}$ with backoff error. 
Table 4.2: Implementation Parameters of PM2M overlay Network

\begin{tabular}{l|l} 
codes per cell, $\mathrm{N}$ & 64 \\
codes for H2H traffic, $N_{i}$ & 46 \\
codes for PM2M overlay traffic, $N_{M}$ & 8 \\
LTE frame duration & 540 overlay backoff periods \\
LTE system bandwidth & $5 \mathrm{MHz}$ \\
PRACH bandwidth & $\mathrm{W}=1.08 \mathrm{MHz}$ \\
preamble length & 839 elements \\
preamble duration & $1600 \mu \mathrm{s}$ \\
preamble elements & $N_{b}=16$ \\
preamble format & 2 \\
RACH configuration index & $c_{f}=5$ \\
preamble element rate & $R_{p} r a t e=1.048 \mathrm{M}$ elements \\
Traffic class & $T C_{0}$ and $T C_{1}$ \\
one backoff period & $18.51 \mu$ s \\
Superframe Beacon interval & 540 backoff periods \\
PM2M superframe duration & 410 backoff period \\
PM2M MAC data packet size & 30 bytes with header \\
Maximum number of attempts to transmit the packet, $\mathrm{R}$ & 7 \\
\hline
\end{tabular}

\subsubsection{Performance without backoff error}

We first investigate the capacity of PM2M overlay network on PRACH by neglecting the error in clear channel assessment. Packet arrival rate per M2M node was set in the range between 0.4 to 2 packets/sec, while the number of PM2M nodes was varied between 240 and 1560. The upper bound for the number of nodes was selected so as to capture reasonable decline in transmission success probability.

Fig. 4.8 shows the probability of successful access to the medium by 2 traffic classes. We could consider higher priority traffic class $T C_{1}$ for vehicular emergency safety messages and lower priority traffic class $T C_{0}$ for crowd sensing. We investigate and found that we can accommodate only 1560 nodes for each traffic classes that can successfully access the medium after completing the random access process. We are expecting that our higher priority traffic class $T C_{1}$ should has higher probability to access the medium than 
lower priority class $T C_{0}$. Fig. $4.8 \mathrm{~b}$ shows for $T C_{1}$ upto 1560 nodes/sec the probability of success is $87 \%$ where as for $T C_{0}$ (Fig. $4.8 \mathrm{a}$ ) it is $85 \%$. The probability of success is decreasing with increase of nodes because of the lack of the availability of the resources. We notice that traffic class 1 achieves 5\% higher success probability than traffic class 0 for 1560 nodes. Fig. 4.9 shows the mean backoff time for $T C_{1}$ (Fig. 4.9 b) up to 1560 nodes it is closer to 35 slots where as for $T C_{0}$ (Fig. 4.9a) it is closer to 98 slots. This is reasonable since lower priority traffic class with higher contention window value has to backoff longer period of time than the higher priority class with shorter contention window. We calculate medium access probability which refers to access the PRACH with parallel to $\mathrm{H} 2 \mathrm{H} 100$ calls/sec without any interference. Fig. 4.10 shows that $T C_{0}$ (figure $4.10 \mathrm{a}$ ) has $15 \%$ lower priority to access the medium than $T C_{1}$ (Fig. 4.10b). We calculate normalized throughput for each node which defined as fraction of time in which the channel is used to transmit the frames payload. Figure $4.11 \mathrm{~b}$ shows that throughput of $T C_{1}$ is 3 times higher than $T C_{0}$ (fig. 4.11a) for $1560 \mathrm{PM} 2 \mathrm{M}$ nodes and highest data arrival rate. In case of number of backoff attempt a node has to backoff before get chance to access the medium is shown in Fig. 4.12 for both traffic classes. Fig. 4.12a shows that lower traffic class $T C_{0}$ has to backoff more number of times than higher priority traffic class $T C_{1}$ (Fig. $4.12 \mathrm{~b}$ ) respectively; again, we note slight disadvantage of traffic class 0 with respect to class 1 .

\subsubsection{Performance comparison with reference approach for without backoff error}

To validate our proposed approach we compare it with [87] where the authors proposed a grouping based overload control mechanism. To overcome the problem of overload and 

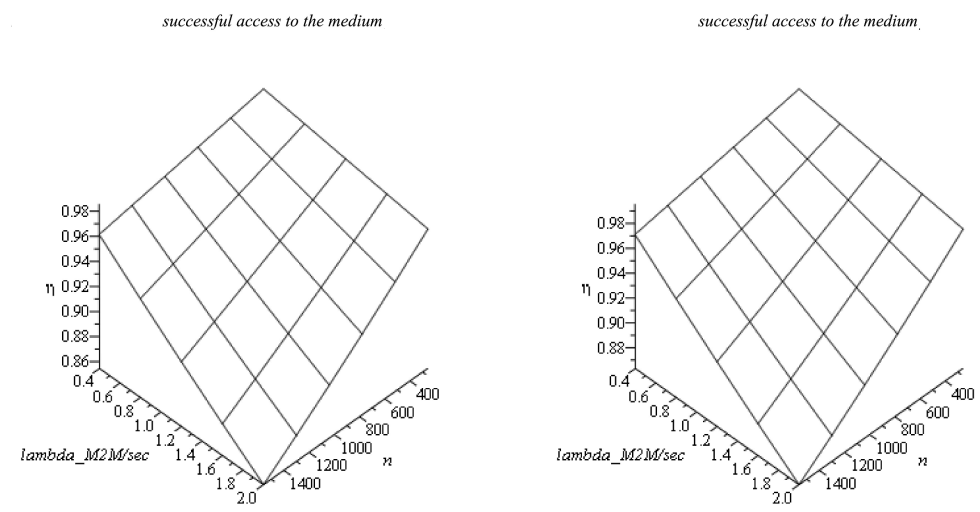

(a) Probability of successful access to(b) Probability of successful access to the medium by each node of class 0 the medium by each node of class 1

Figure 4.8: Probability of successful access to the medium by each node without backoff error.
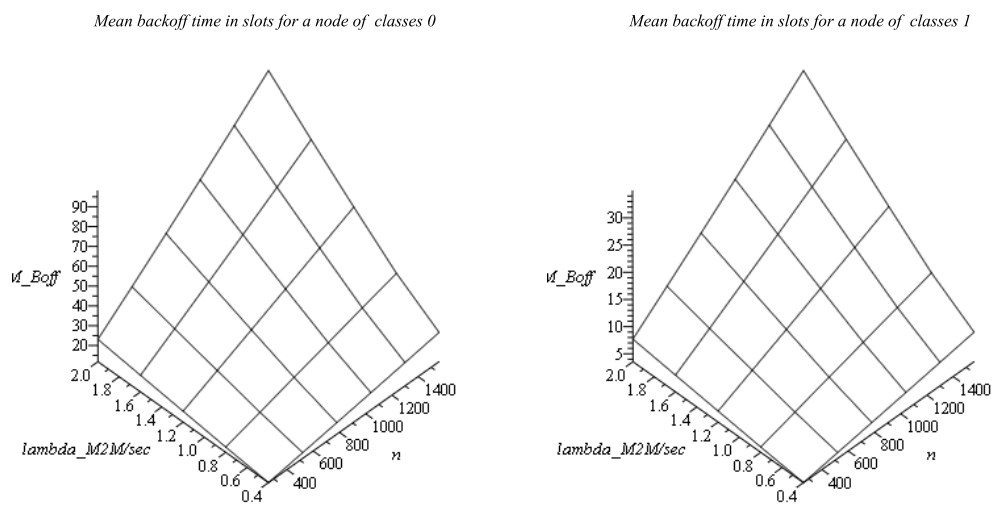

(a) Mean Backoff time of each node of(b) Mean Backoff time of each node of class 0 class 1

Figure 4.9: Mean Backoff time of each node without backoff error.

congestion due to concurrent access attempt by massive M2M devices in their proposed approach group leader initiates RA and apply for transmission resources on behalf of the group members. To avoid signalling congestion base station send only one control signal to the group leader. Second group paging scheme is used for the devices who failed to 

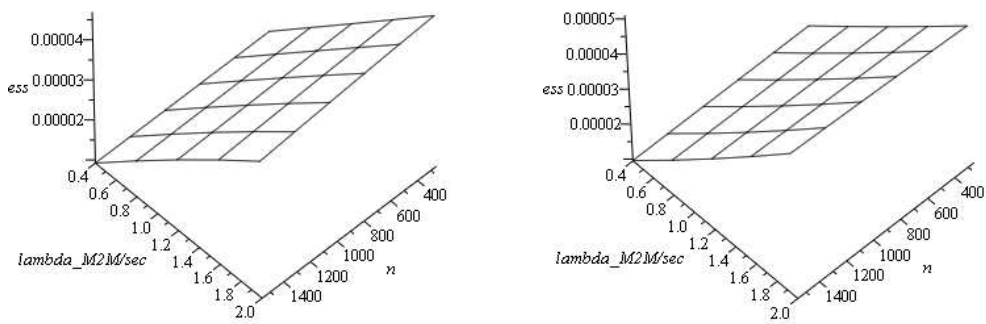

(a) Medium access probability by each(b) Medium access probability by each node of class 0 node of class 1

Figure 4.10: Medium access probability by each node without backoff error.
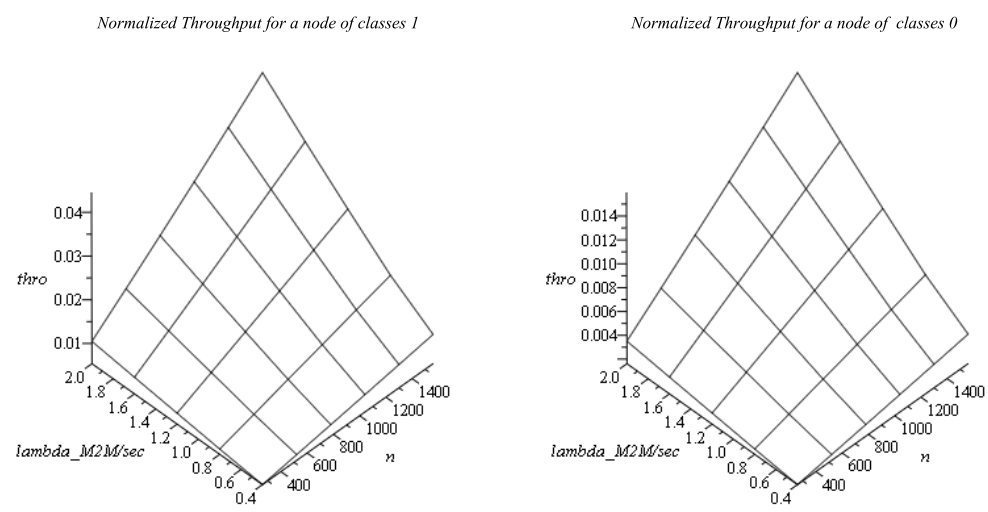

(a) Normalized throughput by each(b) Normalized throughput by each node of class 1 node of class 0

Figure 4.11: Normalized throughput by each node without backoff error.

transmit in current time window. They proposed a five-step handshaking mechanism for RA procedure. The limitation of the proposed approach is it decreases the number of M2M devices which are allowed to access the network. The group leader still needs to follow 5step handshaking mechanism which creates signalling overhead. Selection of group leader 

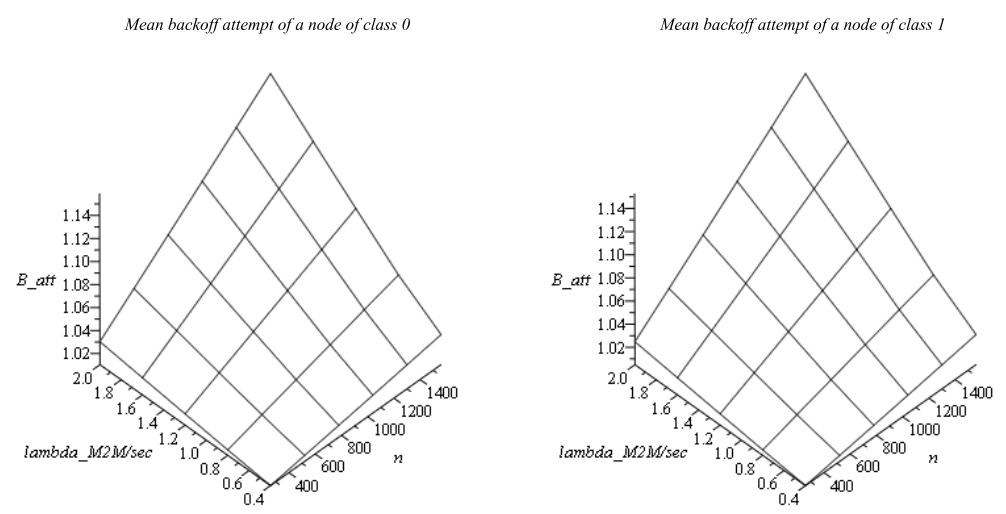

(a) Mean number of backoff attempt by(b) Mean number of backoff attempt each node of class 0 by each node of class 1

Figure 4.12: Mean number of backoff attempt by each node without backoff error.

among M2M devices is the challenging issue for grouping methods. If the size of the group is massive it can cause the probability of collision high results low access success probability and high access delay. It also has the disadvantages of low resource utilization issue compared with our PM2M overlay approach.

Through the experiments (4.6.1) without consideration of the presence of error during the backoff procedure, we have shown that with the presence of $100 \mathrm{H} 2 \mathrm{H}$ calls we can accommodate only 1560 PM2M nodes for both traffic classes without overloading the access networks (Fig. 4.5c). We only compare the reference approach without backoff error scenarios as the reference approach does not consider backoff error during backoff procedure. In this experiment, we fixed the PM2M arrival rate as 2 packets/sec and $\mathrm{H} 2 \mathrm{H}$ intensity was fixed for 100 calls/sec. We consider two traffic classes for PM2M overlay, $T C_{0}$ as lower traffic class and $T C_{1}$ as higher traffic class. For reference approach reff class $\mathrm{A}$ as lower priority and reff class B as higher priority traffic class. We vary the nodes between 240 to 1560 and measure the performance metrics of the probability of success and mean access 
delay. Table 4.3 illustrates the comparison between two approaches.

Table 4.3: Comparison between reference approach and PM2M overlay

\begin{tabular}{|l|l|}
\hline Reference approach & PM2M overlay \\
\hline Pull based overload control mechanism. & Push based overload control mechanism. \\
\hline $\begin{array}{l}\text { Use Slotted ALOHA as medium access } \\
\text { control mechanism. }\end{array}$ & $\begin{array}{l}\text { Use CSMA/CA as medium access control } \\
\text { mechanism. }\end{array}$ \\
\hline Restrict M2M access. & $\begin{array}{l}\text { PM2M devices access in parallel with } \\
\text { H2H traffic with dedicated preamble } \\
\text { codes. }\end{array}$ \\
\hline $\begin{array}{l}\text { Group members priority is defined and } \\
\text { depends on the decision of eNodeB and } \\
\text { change according to scenarios and other } \\
\text { parameters. }\end{array}$ & $\begin{array}{l}\text { IEEE 802.15.6 standard supports priority } \\
\text { among different traffic classes of PM2M } \\
\text { devices. }\end{array}$ \\
\hline $\begin{array}{l}\text { Group leader needs to follow 5 step RA } \\
\text { mechanism and extra second group pag- } \\
\text { ing procedure. }\end{array}$ & $\begin{array}{l}\text { PM2M overlay follows 2 way handshak- } \\
\text { ing mechanism and other H2H traffic fol- } \\
\text { lows LTE conventional RA 4-way hand- } \\
\text { shaking mechanism. }\end{array}$ \\
\hline $\begin{array}{l}\text { Selection of group leader among M2M } \\
\text { devices is the challenging issue for group- } \\
\text { ing methods. }\end{array}$ & $\begin{array}{l}\text { 2-way handshaking reduces overload of } \\
\text { the network. }\end{array}$ \\
\hline $\begin{array}{l}\text { Group paging scheme has the disadvan- } \\
\text { tages of low resource utilization issue. }\end{array}$ & $\begin{array}{l}\text { PM2M overlay use only dedicated num- } \\
\text { ber of preambles and H2H traffic use sep- } \\
\text { arate preambles to access the PRACH. }\end{array}$ \\
\hline $\begin{array}{l}\text { Only consider M2M capacity without } \\
\text { considering impact of H2H traffics access } \\
\text { in parallel. }\end{array}$ & $\begin{array}{l}\text { We consider the capacity of PM2M under } \\
\text { the presence of H2H traffic transmitting } \\
\text { in parallel to PM2M. }\end{array}$ \\
\hline $\begin{array}{l}\text { Among 64 preambles, 30 preambles are } \\
\text { dedicated to M2M traffic without consid- } \\
\text { ering the co-existence issue of both net- } \\
\text { works. }\end{array}$ & $\begin{array}{l}\text { PM2M overlay is implemented only with } \\
8 \text { dedicated codes which allows both net- } \\
\text { works fair co-existence. }\end{array}$ \\
\hline $\begin{array}{l}\text { Access success probability decreases } \\
\text { when the size of the group is massive. }\end{array}$ & $\begin{array}{l}\text { Success probability is high and access de- } \\
\text { lay is less compared with their scheme as } \\
\text { signaling overhead and congestion is re- } \\
\text { duced through 2 way handshaking mech- } \\
\text { anism. }\end{array}$ \\
\hline
\end{tabular}

We evaluate the performance of PM2M overlay and reference approach ( $[86,87])$ by considering $100 \mathrm{H} 2 \mathrm{H}$ calls/sec is transmitting in parallel with both networks and varying 


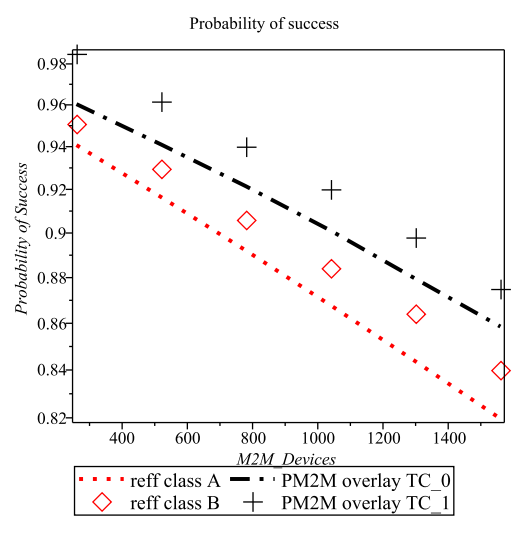

(a) Probability of success

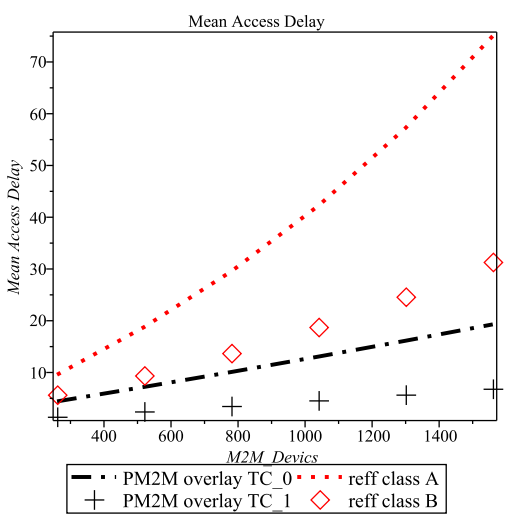

(b) Mean Backoff time

Figure 4.13: Performance evaluation of PM2M overlay and reference approach for without consideration of backoff error

the M2M devices between 240 to 1560 . In reff they did not consider the presence of $\mathrm{H} 2 \mathrm{H}$ calls, so we evaluate the performance to investigate the impact of $\mathrm{H} 2 \mathrm{H}$ traffic over their proposed approach. From Fig. 4.13a we make 4 observations. Firstly, overlay's both traffic classes probability of success is high compared with reff approach. Reference approach considers slotted ALOHA as medium access mechanism whereas, PM2M overlay uses CSMA/CA mechanism which gives advantages over reff approach. Secondly, PM2M overlay both traffic classes follow 2 way handshaking mechanism compared with 5 steps RA mechanism of reff both traffic classes. Reducing the extra steps to send small M2M data gives the benefit over reff approach. Thirdly, Probability of success decreases with the increase of M2M devices as available RA resources are decreasing. fourthly, Under the presence of $\mathrm{H} 2 \mathrm{H}$ traffic reff approach success rate for reff class A and reff class B for $240 \mathrm{M} 2 \mathrm{M}$ devices is only $95 \%$ and $94 \%$ compared with $T C_{1}, T C_{0} 99 \%$ and $96 \%$. PM2M overlay gain 5\% compared with reff approach. From Fig. 4.13b we could observe that using overlay approaches reduces access delay for both traffic classes $T C_{0}$ and $T C_{1}$ 
$10 \%$ compared with reff class A and reff class $\mathrm{B}$. Higher priority traffic class $T C_{1}$ has high priority to access the network and its high probability of success gives it advantages over other traffic classes including $T C_{0}$,reff class $\mathrm{A}$ and reff class $\mathrm{B}$. This is reasonable since lower priority traffic class with higher contention window value has to backoff longer period of time than the higher priority class with shorter contention window.

These results seem comparable to those obtained by other reported schemes $[4,24,87]$. Unlike those schemes, our analysis explicitly models the impact of interference from other random access attempts both in the given and surrounding cells, which means that our results are more realistic. Furthermore, our scheme allows short messages typical for M2M devices to be actually transmitted during random access, whereas all other schemes use random access to initiate connection, as is common for $\mathrm{H} 2 \mathrm{H}$ calls.

\section{Performance evaluation with backoff error}

We evaluate the performance of PM2M with backoff error for both traffic classes under the same conditions as in the previous experiment, except that number of nodes was ranging between 240 and 1320. As before, these values were chose to result in the decrease of the probability of successful access to the medium in the range similar to the case without backoff error, as considered in the previous Subsection. We investigate the impact of backoff error on the capacity of PM2M to access PRACH with parallel to the $\mathrm{H} 2 \mathrm{H}$ traffic. We solved the analytical model of backoff error using Maple 13 [56] and calculate the performance metrics of probability of successful access to the medium, normalized throughput, mean backoff time, medium access probability by each node of UPs.

We found that the capacity of PM2M networks to access the PRACH is lower than the 
experiment without backoff error as shown in Section 4.6.1. We investigate and found that we can accommodate only 1320 nodes for each traffic classes that can successfully access the medium after completing the random access process which represents a $12 \%$ decrease of overlay network capacity. We are expecting that our higher priority traffic class $T C_{1}$ should has higher probability to access the medium than lower priority class $T C_{0}$. Fig. $4.15 \mathrm{a}$ shows for $T C_{1}$ upto 220 nodes/sec the probability of success is $98 \%$ where as for $T C_{0}$ (Fig. 4.14a) it is $94 \%$. Lower priority traffic class with higher contention window value has to backoff longer period of time than the higher priority class which has shorter contention window value. As the number of nodes are increasing up to $1320 T C_{0}$ performance is decreasing close to $83 \%$ where as for traffic class $T C_{1}$ it is only $87 \%$. We calculated the mean number of backoff time in slots and Fig. 4.15b shows the mean backoff time of $T C_{1}$ for highest load is closer to 33 slots where as for $T C_{0}$ (Fig. 4.14b) it is closer to 100 slots. We calculate medium access probability which refers to access the PRACH with parallel to $\mathrm{H} 2 \mathrm{H} 100$ calls/sec without interference. Fig. 4.14c shows that $T C_{0}$ has 10 to $15 \%$ lower priority to access the medium than $T C_{1}$ (Fig. $4.15 \mathrm{c}$ ). We calculate normalized throughput for each node which defined as fraction of time in which the channel is used to transmit the frames payload. Fig. $4.15 \mathrm{~d}$ shows that throughput of $T C_{1}$ is 3 times higher than $T C_{0}$, Fig. 4.14d) under highest load. Regarding the mean number of backoff attempts, Fig. $4.14 \mathrm{e}$ shows that lower traffic class $T C_{0}$ has to perform slightly more backoff attempts than higher priority traffic class $T C_{1}(4.15 \mathrm{e})$ as we expected with the presence of backoff error 


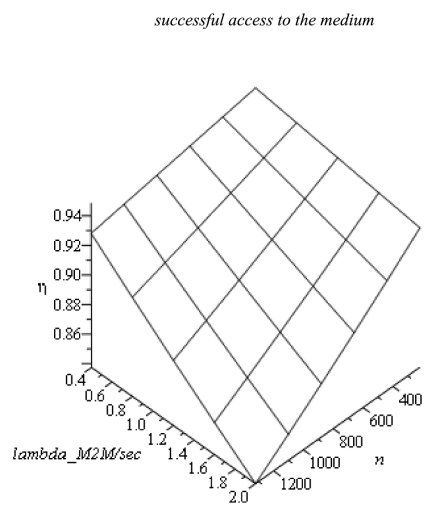

(a) Probability of success

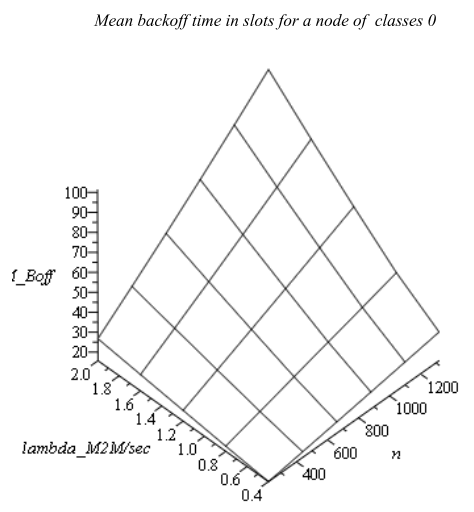

(b) Mean Backoff time

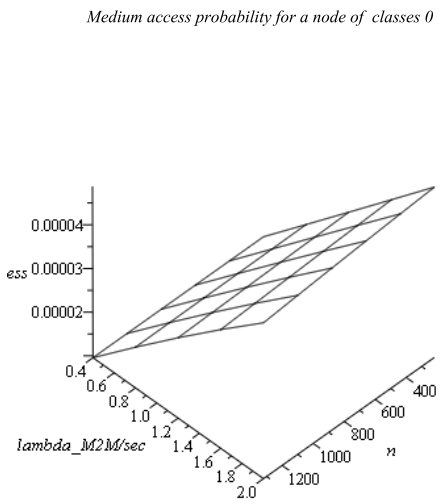

(c) Medium access probability

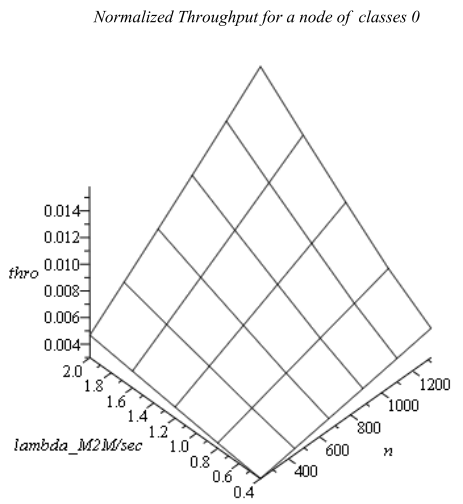

(d) Normalized throughput

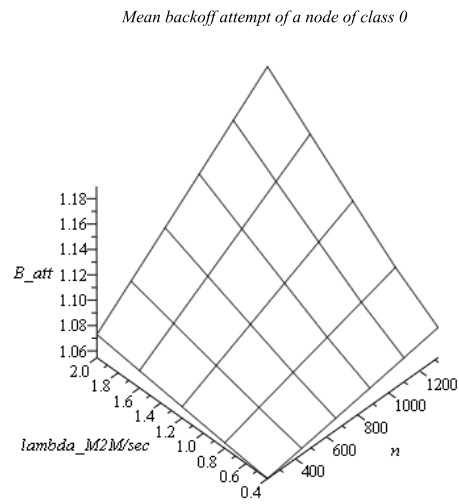

(e) Mean number of backoff attempt

Figure 4.14: Performance evaluation of each node of PM2M overlay for lower traffic class $T C_{0}$ with backoff error

\subsubsection{Summary: Comparison between PM2M overlay network with and without Backoff error in the presence of $\mathrm{H} 2 \mathrm{H}$ traffic}

We compare the capacity of non-priority CSMA/CA VM2M overlay architecture as proposed in chapter 3 with priority based CSMA/CS PM2M overlay. We could see that the later architecture accommodates more node than the previous one. IEEE 802.15.6 supports larger superframe time with $c_{f}=5, \mathrm{PF}=2$ which means that 5 independent PRACH 


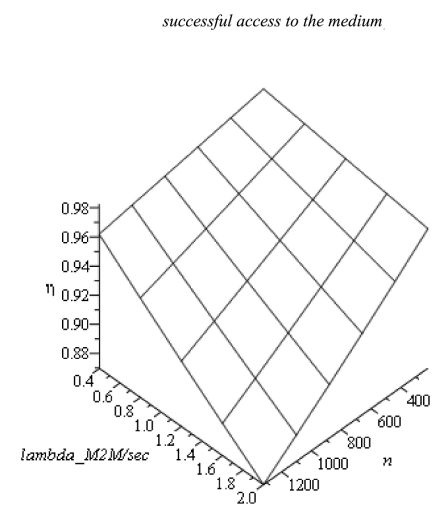

(a) Probability of success

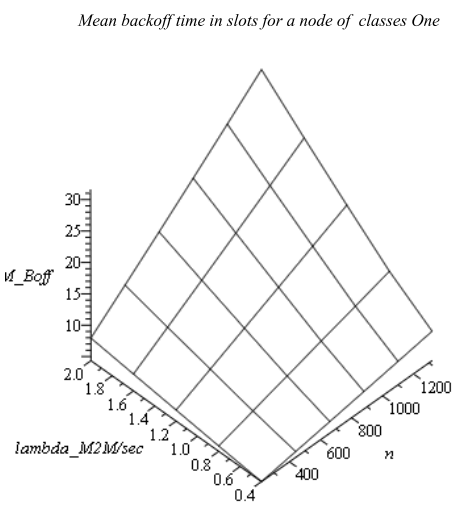

(b) Mean Backoff time

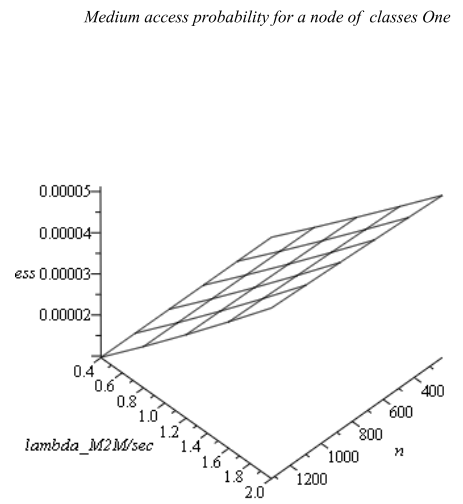

(c) Medium access probability

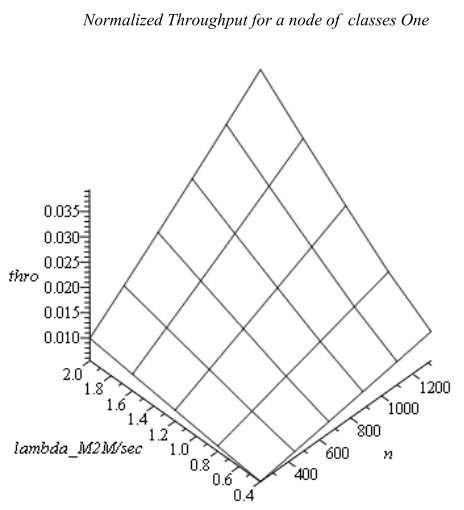

(d) Normalized throughput

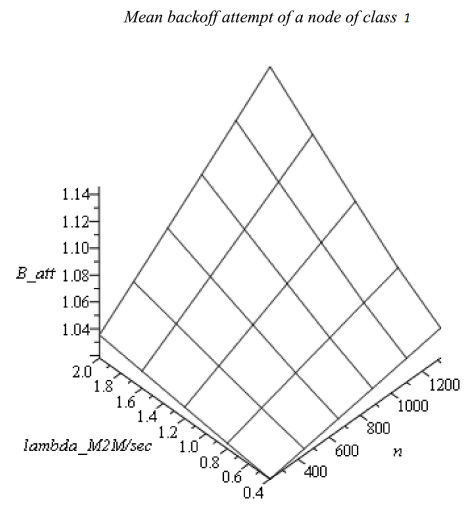

(e) Mean number of backoff attempt

Figure 4.15: Performance evaluation of each node of PM2M overlay for higher traffic class $T C_{1}$ with backoff error

resources are available in one LTE frame. Each PRACH resource has preamble duration of $1600 \mu$ s. It allows to accommodate more PM2M nodes without interfering with $\mathrm{H} 2 \mathrm{H}$ traffic.

The performance of $\mathrm{H} 2 \mathrm{H}$ traffic, Figure 4.5 shows that the PM2M traffic is not overloading and interfering with the $\mathrm{H} 2 \mathrm{H}$ traffic greatly. Our challenge was to ensure that PM2M and $\mathrm{H} 2 \mathrm{H}$ traffic have fair access to the $\mathrm{RACH}$ resources. We investigated the capacity of both subnetworks under various parameters and we have shown that using PM2M 
overlay network allows fair coexistence of PM2M and $\mathrm{H} 2 \mathrm{H}$ traffic.

We consider non-saturation conditions so like saturation our lower priority traffic class is not starving. Both the traffic classes queue can be empty after successful data transmission or dropped due to unsuccessful and exceeded retry limits. From both experiments we investigate that the capacity of PM2M networks with the fixed H2H traffic of 100 calls/sec decreases from 1560 nodes to 1320 . Because of the backoff error and noise in the channel, we could not accommodate more than 1320 nodes to access the PRACH for each of the two traffic classes. By comparing Fig. 4.15a, 4.14a and 4.8 we could see that without any error (Fig. 4.8) the probability of success for $T C_{1}$ (Fig. 4.8b) and $T C_{0}$ (Fig. 4.8a) is more than $98 \%$ with 1560 nodes in each class. Whereas, with error the total capacity decreases to 1320 and the probability of success for $T C_{1}$ (Fig. 4.15a) and $T C_{0}$ (Fig. 4.14a) for up to 220 nodes is less than $95 \%$. Mean backoff time of $T C_{0}$ (Fig. 4.14b) is more than 100 slots for up to 1320 nodes, compared with 97 slots for up to 1560 nodes of $T C_{0}(($ Fig. 4.9 a) without any error. Because of the wrong listing of the medium, a node has to wait for longer backoff period. For all others performance metrics the PM2M without any error outperforms compare with backoff error. Our finding is that for both experiments the higher traffic class $T C_{1}$ is performing better than lower traffic class $T C_{0}$.

\subsection{Chapter summary}

In this chapter, we have presented an IEEE 802.15.6-based overlay network which allows the LTE network to support massive M2M traffic with priorities over PRACH. The PM2M overlay operates by dedicating a number of available preambles to the physical layer of the overlay network; the remaining preambles can be used for LTE-prescribed ran- 
dom access by regular LTE UEs. We have modelled the performance of the overlay as well as the mutual interference of $\mathrm{H} 2 \mathrm{H}$ and PM2M traffic when both are present in the LTE cell, and the performance of PM2M overlay with and without the errors in the clear channel sense performed during the backoff countdown. The scheme considers the backoff error during CSMA/CA mechanism coming from the physical layer of $\mathrm{H} 2 \mathrm{H}$ traffic. We develop a 3 dimensional Markov chain in order to model the backoff procedure of CSMA/CA to investigate the effect of the error in physical and MAC layer with the presence of $\mathrm{H} 2 \mathrm{H}$ traffic.

We evaluate the performance through a detailed analytical model and evaluates the impact of error on the capacity of the overlay network. Our results indicate, first, that the $\mathrm{H} 2 \mathrm{H}$ traffic still enjoys fair access to the PRACH despite the presence of the PM2M overlay. Second, the PM2M overlay is capable of accommodating up to about 1500 M2M devices in a single cell with default LTE capacity. Third, the backoff error reduces this capacity by about $12 \%$ compared to the case with perfect channel sensing. In both cases, the PM2M overlay is capable of providing sufficient differentiation between low- and high priority traffic classes. given backoff period. Our results show that including backoff error decreases overlay capacity by approximately $12 \%$ compared to the perfect clear channel assessment. We have also evaluated priority differentiation in overlay network using different sizes of backoff windows. Therefore, we could consider higher priority traffic class for more critical M2M applications such as vehicular safety, and lower priority traffic class for applications with less stringent requirements such as crowd sensing, thus making it suitable for a wide range of smart city applications.

To validate the proposed approach we compare the proposed scheme with a reference 
scheme. We have shown that our approach outperforms than reference approach in the respect of the probability of success and mean access delay. 


\section{Chapter 5}

\section{Conclusion}

In this dissertation, we have investigated the application areas of machine to machine (M2M) communications specifically in the area of smart city to build smart intelligent transportation systems, smart healthcare systems, smart parking, smart street lighting. We conduct the case study for non-priority CSMA/CA and priority-based CSMA/CA scenarios. First, we consider VANET as a case study and propose a novel non-priority $\mathrm{CCH} / \mathrm{SCH}$ architecture over LTE which allows for an efficient implementation of a VANET, with particular attention to areas with large distances and higher vehicle speed. The proposed architecture uses LTE PRACH as the underlying transport for a vehicular M2M (VM2M) overlay network. The $\mathrm{CCH}$ architecture is implemented using a dedicated subset of available preambles at the physical layer, and a carrier sense multiple access with collision avoidance (CSMA/CA) mechanism similar to the one used in IEEE 802.15.4 low data rate WPAN at the medium access control layer.

Depending on the cell size, vehicle speeds and traffic condition VM2M can be implemented using different preamble formats $(\mathrm{PF})$ and configuration index $\left(c_{f}\right)$. By experiments 
with deployment scenarios and analytical model, we have shown that the overlay works allows fair coexistence of VM2M and $\mathrm{H} 2 \mathrm{H}$ traffic. The choice of preamble format for $\mathrm{H} 2 \mathrm{H}$ and VM2M depends on the environment. We wanted to maximize the capacity of VM2M but cannot sacrifice the $\mathrm{H} 2 \mathrm{H}$ traffic. For sub-urban, rural and highway scenarios RACH format 2 or even 3 might be necessary due to large velocity and round trip time. For urban environment $\mathrm{RACH}$ format 0 can be used and capacity can be increased with increasing $\mathrm{RACH}$ configuration parameter. The use of overlay network for the $\mathrm{CCH}$ affects $\mathrm{H} 2 \mathrm{H}$ traffic with the deterministic impact of $N_{M}$ active preambles. On the other hand variable rates of $\mathrm{H} 2 \mathrm{H}$ requests present variable (random) interference to VM2M overlay. We have shown that with $N_{M}=8$ dedicated preamble codes for VM2M overlay, both LTE and VM2M networks can have fair coexistence. In the scenarios of transmitting safety messages of VM2M with a higher power, we evaluate the impact of human to human $(\mathrm{H} 2 \mathrm{H})$ layer over VM2M overlay when VM2M gives priority to the physical layer to access the base station (eNodeB). We showed that VM2M traffics are so strong with priority that with $220 \mathrm{H} 2 \mathrm{H}$ calls/s still, we could have 1000 OBUs in the subnetwork.

IEEE 802.15.4 do not support high data rate and priority among different traffic classes. To transmit emergency data with high data rate, in real time and to introduce priority among different traffic classes we adopted IEEE 802.15.6 as transmission protocol and propose a priority based machine to machine (PM2M) overlay network over LTE. We analyze the architecture with and without backoff error and investigate the capacity of PM2M traffic with the presence of $\mathrm{H} 2 \mathrm{H}$ traffic. We propose the model of probability of backoff error coming from the physical layer of $\mathrm{H} 2 \mathrm{H}$, which is more realistic. We propose a 3 dimensional Discrete Time Markov chain (DTMCs) to model the backoff procedure of the CSMA/CA 
mechanism with the probability of backoff error only for two traffic classes and random access phase (RAP). We investigate the impact of error on the capacity of PM2M to access PRACH with parallel to the $\mathrm{H} 2 \mathrm{H}$ traffic. We showed that higher priority traffic class as expected outperform over lower traffic class even with the backoff error while $\mathrm{H} 2 \mathrm{H}$ traffics are fixed for 100 calls/sec. By comparing the experiments of with and with backoff error we investigate that the capacity of PM2M networks decreases from 1560 nodes to 1320 nodes. Because of the backoff error and noise in the channel, we could not accommodate more than 1320 nodes to access the PRACH for each of the two traffic classes. By comparing the capacity of VM2M overlay architecture with priority based PM2M overlay, we could see that the later architecture accommodates more node than the previous one.

To validate our PM2M overlay network we compare the proposed approach with current reference approach. By performance evaluation, we have shown that PM2M overlay outperforms compared with reference approach. The PM2M overlay has the advantages of using priority based CSMA/CA mechanism and reduced 2-way handshaking mechanism compared to slotted ALOHA and 5 steps RA handshaking mechanism.

In this dissertation, we only investigate the M2M applications in the area of smart city. As a future work, we would like to work on other areas like smart grids, home area networks, environmental monitoring and industrial automation. In PM2M we only consider 2 access categories (AC) and only random access phase. However, the standard allows 7 AC's and other access phases such as Exclusive access phase (EAP 1 and EAP 2), Type-I/II access phase and Contention access phase (CAP). In future, we would like to consider all the 7 ACs for our PM2M overlay network with EAP and CAP.

M2M communications ubiquitous application scenarios make it leading facilitator for 
internet of things (IoT) paradigm. The increased growth of the mobile internet use has initiated to achieving the fifth generation $(5 \mathrm{G})$ milestone. M2M and IoT are considerated an important part for the future success of $5 \mathrm{G}$ communications. 5G`s multiple input multiple output (MIMO) system and use of small cells characteristics will be able to elevate the capacity, resource, speed and reliability constraints of M2M communications [57]. M2M communications using 5G will overcome the current limitations and by integrating both technologies would yield positive results. As a future work, we will update our proposed overlay networks with $5 \mathrm{G}$ communications for wide deployment scenarios of the smart city. 


\section{Appendix A}

\section{Publications}

\section{A.1 Journal Papers}

[1] N. khan, J. Mišić, and V. B. Mišić. Priority based machine to machine (PM2M) overlay networks over LTE for smart city, In MDPI Journal of Sensor and Actuator Networks, 2018.

[2] N. Khan, J. Mišić, and V. B. Mišić. On Coexistence of Vehicular Overlay Network and H2H Terminals on PRACH in LTE, In ZTE communications, 14(3):1673-5188, August, 2016.

[3] J. Mišić, V. B. Mišić, and N. khan Sharing It My Way: Efficient M2M Access in LTE/LTE-A Network, In IEEE Transactions on Vehicular Technology, January, 2017. 


\section{A.2 Conference Papers}

[1] N. khan, J. Mišić, and V. B. Mišić. IEEE 802.15.6 based LTE overlay network with priority support, In IEEE International Conference on Green Computing and Communications, Halifax, Canada, July, 2018.

[2] N. khan, J. Mišić, and V. B. Mišić. VM2M: An overlay network to support Vehicular traffic over LTE, In International Wireless Communications and Mobile Computing Conference (IWCMC), Paphos, Cyprus, 2016.

[3] N. khan, J. Mišić, and V. B. Mišić. Priority based VM2M communications over LTE, In IEEE 21st International Workshop on Computer Aided Modelling and Design of Communication Links and Networks (CAMAD), Toronto, ON, 2016.

[4] N. khan, J. Mišić, and V. B. Mišić. Tradeoffs in PRACH Bandwidth Partitioning for VM2M Overlay Network in LTE, In IEEE 84th Vehicular Technology Conference (VTC-Fall), Montreal, QC, 2016.

[5] J. Mišić, N. khan, H. Khojasteh and V. B. Mišić. CSCD: a simple channel scan protocol to discover and join a cognitive PAN, In the IEEE Wireless Communications and Networking Conference on Communications (WCNC 2012), Paris, France, April 2012.

[6] J. Mišić, H. Khojasteh, N. khan and V. B. Mišić. Towards an efficient rendezvous protocol for a cognitive PAN, In the IEEE International Conference on Communications (ICC 2012), Ottawa, Canada, June 2012.

[7] N. khan, J. Mišić, V. B. Mišić, and L. karim. Providing QoS in the integration of 
RFID and Wi-Fi WLAN, In International Conference on Ad hoc Networks and Wireless (Adhoc Now), Belgrade, July 2012. 


\section{Appendix B}

\section{Abbreviations and Symbols}

\section{B.1 Abbreviations}

$\begin{array}{ll}\text { AC } & \text { Access Category } \\ \text { ACK } & \text { Acknowledgement } \\ \text { AP } & \text { Access Point } \\ \text { APs } & \text { Access Phase } \\ \text { BC } & \text { Backoff Counter } \\ \text { BE } & \text { Backoff Exponent } \\ \text { BER } & \text { Backoff Error } \\ \text { BI } & \text { Beacon Interval } \\ \text { BO } & \text { Beacon Order } \\ \text { CAP } & \text { Contention Access Point } \\ \text { CCA } & \text { Clear Channel Assessment }\end{array}$




\begin{tabular}{ll} 
CCH & Control Channel \\
CP & Cyclic Prefix \\
CRNTI & Cell Radio Network Temporary Identifier \\
CSMA & Carrier Sense Multiple Access \\
CSMA/CA & Carrier Sense Multiple Access/ Collision Avoidance \\
CW & Contention Window \\
EAP & Exclusive access Phase \\
FCS & Frame Check Sequence \\
FDD & Frequency division Duplex \\
GT & Guard Time \\
H2H & Human to human \\
HBC & Human Body Communication \\
ISM & Industrial Scientific and Medical \\
LTE & Long Term Evolution \\
M2M & Machine to Machine \\
MAC & Medium Access Control \\
MT & Mobile terminals \\
MTC & Machine Type Communications \\
NB & Narrow Band \\
OBU & On Board unit \\
\hline OFD & Orthogonal frequency division Multiplexing \\
\hline
\end{tabular}




\begin{tabular}{|c|c|}
\hline PHY & Physical Layer \\
\hline $\mathrm{PF}$ & Preamble Fomat \\
\hline PRACH & Physical Random Access Channel \\
\hline pdf & Probability Density Function \\
\hline PDF & Probability Distribution Function \\
\hline PGF & Probability Generating Function \\
\hline PPDU & Physical layer Protocol Data Unit \\
\hline PSDU & Physical layer service data Unit \\
\hline PS & Personal Server \\
\hline PUSH & Physical Uplink Shared Channel \\
\hline QoS & Quality of Service \\
\hline RA & Random Access \\
\hline $\mathrm{RACH}$ & Random Access Channel \\
\hline RAP & Random Access Phase \\
\hline RAR & Random Access Respons \\
\hline $\mathrm{RB}$ & Resource Block \\
\hline $\mathrm{RE}$ & Resource Element \\
\hline $\mathrm{RBC}$ & Random Backoff Countdown \\
\hline RSU & Road Side unit \\
\hline SAP & Service Access Point \\
\hline SAR & Specific Absorption rate \\
\hline
\end{tabular}




\begin{tabular}{|c|c|}
\hline $\mathrm{SCH}$ & Service Channel \\
\hline SIFS & Short Interframe Space \\
\hline $\mathrm{TC}$ & Traffic Class \\
\hline TDD & Time Division Duples \\
\hline TDMA & time division Multiple Access \\
\hline UP & User Priority \\
\hline UWB & Ultra Wide Band \\
\hline UE & User Equipment \\
\hline VANET & Vehicular Ad hoc Network \\
\hline $\mathrm{V} 2 \mathrm{~V}$ & Vehicle to Vehicle \\
\hline V2R & Vehicle to Roadside \\
\hline VM2M & Vehicular Machine to Machine \\
\hline WAVE & Wireless Access for the Vehicular Environment \\
\hline WBAN & Wireless Body Area Network \\
\hline WBM2M & Wireless Body Area Machine to Machine \\
\hline WMTS & Wireless Medical Telemetry Services \\
\hline $\mathrm{ZC}$ & Zadoff-Chu \\
\hline
\end{tabular}

\section{B.2 Symbols and Corresponding Descriptions}

PF $\quad$ Preample format 


$\begin{array}{ll}N & \text { Total number of preambles } \\ N_{h} & \text { Preambles for handover calls } \\ N_{M} & \text { Number of ZC preambles in physical layer of VM2M or PM2M networks } \\ C W_{\text {min }} & \text { Contention window value minimum } \\ C W_{\text {max }} & \text { Contention window value maximum } \\ c_{f} & \text { Configuration index determines how many PRACH resources } \\ t_{b o f f} & \text { occur in one LTE frame } \\ N_{b} & \text { backoff time slot } \\ T_{f} & \text { Duration of M2M data bit running over the preamble } \\ \lambda_{w} & \text { LTE superframe time } \\ E_{s e q} & \text { Mean arrival rate of h2H traffic } \\ N_{0} & \text { Preamble sequence energy } \\ P_{s e q} & \text { Noise power density } \\ W & \text { preamble sequence length in bits } \\ R & \text { LTE bandwidth } \\ I_{1} & \text { LTE PRACH preamble data rate } \\ \eta_{p, 1} & \text { Received signal power } \\ \eta_{0} & \text { The PRACH overload for third handshake step for } j \text { number of H2H traffic } \\ I_{-} P R A C H_{n} & \end{array}$


$\lambda_{\text {total }} \quad$ The total Poisson arrival rate of per PRACH for all the traffic

$P_{O, 1} \quad$ The total overload probability for first handshake step

due to collision and interference

$P_{3}(j) \quad$ The third handshake step collision probability

$P_{Q} \quad$ The overall PRACH overload probability for third handshake step

$P_{\text {M_oload }} \quad$ The total VM2M overload probability

$B E R_{V M 2 M} \quad$ Bit error rate of VM2M

$E_{s} / N_{0} \quad$ Energy per symbol to noise power spectral density

$E_{p} / N_{0} \quad$ Preamble sequence energy to noise power density per $\mathrm{Hz}$

$R \quad$ Maximum number of retry limit

$\sigma \quad$ The probability of neither the data nor the acknowledgement

(ack) is corrupted by noise

$L T E_{B E R} \quad$ BER coming from $\mathrm{H} 2 \mathrm{H}$

data $_{s} \quad$ Data size in slots

ack $\quad$ Acknowledgement size in slots

$P B_{\text {err }} \quad$ The probability of backoff error

$B \quad$ Physical bit

$m_{i} i d l e_{A e r r} \quad$ The wrong medium idle probability

$n \quad$ Number of nodes in each traffic class

m_busy $\quad$ The busy medium probability

$m_{-} e r r_{k} \quad$ The probability of medium idle during backoff countdown 
$\tau_{k} \quad$ Medium access probability

$S_{-} t_{i m e} \quad$ The probability that there is not enough time to complete the current transmissions

$T_{\text {succ }} \quad$ Successful transmission time in slots

$T_{\text {coll }} \quad$ Unsuccessful transmission time in slots

$X_{R A P} \quad$ Random access phase

$h_{k, j} \quad$ The probability that backoff counter value of a node decrements

when the value is equal to $j$

$\operatorname{Ebf~succk,j}_{k}(z)$ the moments between the locking and unlocking the backoff counter due to successful transmission by another node

$\operatorname{Ebfcoll}_{k, j}(z) \quad$ the moments for unsuccessful transmission

$\operatorname{Ebfploc}_{k, j}(z)$ the moments between locking and unlocking when there is not enough time for completing a frame transaction

$P_{\text {succ }, k} \quad$ probability to lock the backoff counter due to successful transmission by others

$P_{\text {coll }, k} \quad$ probability to lock the backoff counter due to unsuccessful transmission by others

$L_{t} \quad$ number of CSMA slots when backoff counter must be locked because not enough time for completing transaction

$b_{a t t m p, k} \quad$ Number of backoff attempt

$B_{-} N_{a t t m p, k} \quad$ Mean number of backoff attempt 
$Z_{b o f f, k} \quad$ Zero-th backoff plase stage

$\alpha \quad$ The probability of data frame arrives during the interval of

between two successive Markov points

$Q_{e m t \quad \text { probability of queue empty }}$

$S_{\text {idle }, k} \quad$ The probability of being in idle state 


\section{Bibliography}

[1] S. Abbas, S. Chaudhry, and G. Yasin. VANET route selection in urban/rural areas using metric base traffic analysis. In UBICOMM 2013, The Seventh International Conference on Mobile Ubiquitous Computing, Systems, Services and Technologies, pages $85-91,2013$.

[2] H. Abid, T. C. Chung, S. Lee, and S. Qaisar. Performance analysis of LTE smartphones-based vehicle-to-infrastrcuture communication. In Ubiquitous Intelligence Computing and 9th International Conference on Autonomic Trusted Computing (UIC/ATC), 2012 9th International Conference on, pages 72-78, Sept 2012.

[3] A. M. Ahmad, Z. Bazzal, A. Kalakesh, and L. A. Wardani. A reliable broadcast approach for VANET's safety messages. In 2017 29th International Conference on Microelectronics (ICM), pages 1-5, Dec 2017.

[4] M. S. Ali, E. Hossain, and D. I. Kim. LTE/LTE-A random access for massive machine-type communications in smart cities. CoRR, abs/1608.03042, 2016.

[5] M. Anwer and C. Guy. A survey of VANET technologies. Journal of Emerging Trends in Computing and Information Sciences, 5(9):661-671, 2014. 
[6] D. Aragao, D. Vieira, M. F. de Castro, and Y. Ghamri-Doudane. A dynamic backoff approach to control the congestion in M2M communication over LTE networks. In 2015 Global Information Infrastructure and Networking Symposium (GIIS), pages $1-6$, Oct 2015 .

[7] G. Araniti, C. Campolo, M. Condoluci, A. Iera, and A. Molinaro. LTE for vehicular networking: a survey. IEEE Communications Magazine, 51(5):148-157, May 2013.

[8] A. Arora, N. Rakesh, and K. K. Mishra. Analysis of safety applications in VANET for LTE based network. In G. M. Perez, K. K. Mishra, S. Tiwari, and M. C. Trivedi, editors, Networking Communication and Data Knowledge Engineering, pages 141154, Singapore, 2018. Springer Singapore.

[9] A. W. Astrain, H.-B. LI, and R. Kohno. Standardization for body area networks. IEICE Transactions on Communications, E92.B(2):366-372, 2009.

[10] M. E. Azhari, A. Toumanari, and R. Latif. Performance analysis of IEEE 802.15.6 and IEEE 802.15.4 for wireless body sensor networks. In 2014 International Conference on Multimedia Computing and Systems (ICMCS), pages 910-915, April 2014.

[11] G. Badawy, J. Mišić, T. Todd, and D. Zhao. Performance modeling of safety message delivery in vehicular ad hoc networks. In 2010 IEEE 6th International Conference on Wireless and Mobile Computing, Networking and Communications, pages 188-195, Oct 2010 .

[12] A. Bhatt and N. Purohit. Performance evaluation of urban and rural areas for VANET. International Journal of Technology Research and Management, 1(5), 2014. 
[13] Y. Bian, M. Song, and X. Zhang. An ultra-low-power medium access control protocol for body sensor network. In 2015 IEEE International Conference on Cyber Technology in Automation, Control, and Intelligent Systems (CYBER), pages 76-81, June 2015.

[14] B. Blaszczyszyn, P. Muhlethaler, and S. Banaouas. A comparison of Aloha and CSMA in Wireless Ad-Hoc Networks under Different Channel Conditions. working paper or preprint, 2010.

[15] IEEE Standard for Local and metropolitan area networks - Part 15.6: Wireless Body Area Networks, Feb 2012.

[16] C. Campolo and A. Molinaro. Data rate selection in WBSS-based IEEE 802.11p WAVE vehicular ad hoc networks. In Communication Systems Networks and Digital Signal Processing (CSNDSP), 2010 7th International Symposium on, pages 412-416, July 2010.

[17] T. Castel, S. Lemey, S. Agneessens, P. V. Torre, H. Rogier, and C. Oestges. LTE as a potential standard for public safety indoor body-to-body networks. In 2015 IEEE Symposium on Communications and Vehicular Technology in the Benelux (SCVT), pages 1-6, Nov 2015.

[18] M. Chen, S. Gonzalez, A. Vasilakos, H. Cao, and V. C. M. Leung. Body area networks: A survey. Mobile Networks and Applications, 16(2):171-193, Apr 2011.

[19] M. Chen, D. O. Mau, X. Wang, and H. Wang. The virtue of sharing: Efficient content delivery in wireless body area networks for ubiquitous healthcare. In 2013 IEEE 
15th International Conference on e-Health Networking, Applications and Services (Healthcom 2013), pages 669-673, Oct 2013.

[20] W. Chen, B. Benoit, F. Hu, T. Jiang, and Q. Wang. Wireless body-area networks, 2015.

[21] Y. Chen and W. Wang. Machine-to-Machine communication in LTE-A. In 2010 IEEE 72nd Vehicular Technology Conference - Fall, pages 1-4, Sept 2010.

[22] J. P. Cheng, C. h. Lee, and T. M. Lin. Prioritized random access with dynamic access barring for RAN overload in 3GPP LTE-A networks. In 2011 IEEE GLOBECOM Workshops (GC Wkshps), pages 368-372, Dec 2011.

[23] R. G. Cheng, F. M. Al-Taee, J. Chen, and C. H. Wei. A dynamic resource allocation scheme for group paging in lte-advanced networks. IEEE Internet of Things Journal, 2(5):427-434, Oct 2015.

[24] S. Cherkaoui, I. Keskes, H. Rivano, and R. Stanica. LTE-A random access channel capacity evaluation for M2M communications. In 2016 Wireless Days (WD), pages 1-6, March 2016.

[25] S. Choi, W. Lee, D. Kim, K. J. Park, S. Choi, and K. Y. Han. Automatic configuration of random access channel parameters in lte systems. In 2011 IFIP Wireless Days (WD), pages 1-6, Oct 2011.

[26] E. Dahlman, S. Parkvall, and J. Skold. 4G: LTE/LTE-Advanced for Mobile Broadband. Academic Press, 1st edition, 2011. 
[27] V. Dhilipkumar, D. Kandar, and C. Sarkar. Enhancement of inter-vehicular communication to optimize the performance of 3G/4G-VANET. In Optical Imaging Sensor and Security (ICOSS), 2013 International Conference on, pages 1-5, July 2013.

[28] M. Y. Elsalamouny, H. H. Halawa, R. M. Daoud, and H. H. Amer. Performance of fault tolerant wban for healthcare. In 2017 International Conference on Engineering MIS (ICEMIS), pages 1-5, May 2017.

[29] H. Fourati, S. Khssibi, T. Val, H. Idoudi, A. Van Den Bossche, and L. Azzouz Saidane. Comparative study of IEEE 802.15.4 and IEEE 802.15.6 for WBAN-based CANet. In 4th Performance Evaluation and Modeling in Wireless Networks (PEMWN 2015), pages pp. 1-7, Hammamet, Tunisia, Nov. 2015.

[30] N. Golmie, D. Cypher, and O. Rebala. Performance analysis of low rate wireless technologies for medical applications. Computer Communications, 28(10):1266 $1275,2005$.

[31] Z. Hameed Mir and F. Filali. LTE and IEEE 802.11p for vehicular networking: a performance evaluation. EURASIP Journal on Wireless Communications and Networking, 2014(1):89, May 2014.

[32] E. Hossain, G. Chow, V. C. M. Leung, R. D. McLeod, J. Mišić, V. W. S. Wong, and O. Yang. Vehicular telematics over heterogeneous wireless networks: A survey. Comput. Commun., 33(7):775-793, May 2010.

[33] L. Hu, O. M. Dung, Q. Liu, T. Han, and Y. Sun. Integration of wireless body area 
networks WBANs and WAN, WIMAX and LTE. KSII Transactions on Internet and Information Systems, 7(5):980-997, May 2013.

[34] B. Y.-S. Huang. Random access procedure for machine type communiecatio in mobile networks. Master's thesis, Czech Technical University, Prague, 2017.

[35] A. K. Jacob and L. Jacob. Enhancing reliability of emergency traffic in IEEE 802.15.6 wireless body area networks. In 2017 IEEE Region 10 Symposium (TENSYMP), pages 1-5, July 2017.

[36] X. Jian, Y. Jia, X. Zeng, and J. Yang. A novel class-dependent back-off scheme for machine type communication in LTE systems. In 2013 22nd Wireless and Optical Communication Conference, pages 135-140, May 2013.

[37] P. Johansson, M. Kazantzidis, R. Kapoor, and M. Gerla. Bluetooth: an enabler for personal area networking. IEEE Network, 15(5):28-37, Sep 2001.

[38] E. Jovanov, A. Milenkovic, C. Otto, and P. C. de Groen. A wireless body area network of intelligent motion sensors for computer assisted physical rehabilitation. Journal of NeuroEngineering and Rehabilitation, 2(1):6, Mar 2005.

[39] J.Y.Khan and M.R.Yuce. Wireless body area network (WBAN) for medical applications. In Intech, January 2010.

[40] H. Karvonen, M. Hmlinen, J. Iinatti, and C. Pomalaza-Rez. Coexistence of wireless technologies in medical scenarios. In 2017 European Conference on Networks and Communications (EuCNC), pages 1-5, June 2017. 
[41] N. Khan, J. Mišić, and V. B. Mišić. Priority based VM2M communications over LTE. In 2016 IEEE 21 st International Workshop on Computer Aided Modelling and Design of Communication Links and Networks (CAMAD), pages 177-182, Oct 2016.

[42] N. Khan, J. Mišić, and V. B. Mišić. VM2M: An overlay network to support vehicular traffic over LTE. In 2016 International Wireless Communications and Mobile Computing Conference (IWCMC), pages 13-18, Sept 2016.

[43] Y. Kim, S. Lee, and S. Lee. Coexistence of Zigbee-based WBAN and WiFi for health telemonitoring systems. IEEE Journal of Biomedical and Health Informatics, 20(1):222-230, Jan 2016.

[44] K. S. Kwak, S. Ullah, and N. Ullah. An overview of IEEE 802.15.6 standard. In 2010 3rd International Symposium on Applied Sciences in Biomedical and Communication Technologies (ISABEL 2010), pages 1-6, Nov 2010.

[45] A. Laya, L. Alonso, and J. Alonso-Zarate. Is the random access channel of LTE and LTE-A suitable for M2M communications? A survey of alternatives. IEEE Communications Surveys Tutorials, 16(1):4-16, First 2014.

[46] W. Li, X. Ma, J. Wu, K. S. Trivedi, X. L. Huang, and Q. Liu. Analytical model and performance evaluation of long-term evolution for vehicle safety services. IEEE Transactions on Vehicular Technology, 66(3):1926-1939, March 2017.

[47] A. Lo, Y. Law, M. Jacobsson, and M. Kucharzak. Enhanced LTE-Advanced randomaccess mechanism for massive Machine-to-Machine (M2M) communications. In Proceedings of the 27th Meeting of Wireless World Research Form (WWRF), Oct 2011. 
[48] 3GPP TSG WG2 71bis R2-104663 LTE: MTC LTE simulations, Aug 2010.

[49] IEEE Standard for Local and metropolitan area networks -Part 15.4: Low-Rate Wireless Personal Area Networks (LR-WPANs), Sept 2011.

[50] A. S. Mahmood, E. Jafer, S. Hussain, and X. Fernando. Wireless body area network development for remote patient health observing. In 2017 IEEE Canada International Humanitarian Technology Conference (IHTC), pages 26-31, July 2017.

[51] M. R. Mardani, S. Mohebi, B. Maham, and M. Bennis. Delay-sensitive resource allocation for relay-aided M2M communication over LTE-advanced networks. In 2017 IEEE Symposium on Computers and Communications (ISCC), pages 1033-1038, July 2017.

[52] ZTE, R2-104662: MTC simulation results with specific solutions, 2010.

[53] 3GPP ts 36.321 v10.1.0. Medium Access Control (MAC) protocol specifications, March 2011.

[54] 3GPP, R2-100182, Access control of MTC devices, CATT,RAN2 68bits, Jan 2010.

[55] 3GPP, R2-104870, Pull based RAN overload control, Huawei and China Unicom ,RAN2 71bits, Aug 2010.

[56] Maplesoft,Inc. Maple 16. Waterloo, ON, Canada, 2013.

[57] Y. Mehmood, N. Haider, M. Imran, A. Timm-Giel, and M. Guizani. M2M Communications in 5G: State-of-the-art architecture, recent advances, and research challenges. IEEE Communications Magazine, 55(9):194-201, 2017. 
[58] J. Mišić, G. Badawy, and V. B. Mišić. Performance characterization for IEEE 802.11p network with single channel devices. IEEE Transactions on Vehicular Technology, 60(4):1775-1787, May 2011.

[59] J. Mišić and V. B.Mišić. Wireless Personal Area Networks: Performance, Interconnection and Security with IEEE 802.15.4. John Wiley and Sons, 2008.

[60] J. Mišić and V. B. Mišić. Bridging between IEEE 802.15.4 and IEEE 802.11b networks for multiparameter healthcare sensing. IEEE Journal on Selected Areas in Communications, 27(4):435-449, May 2009.

[61] J. Mišić, V. B. Mišić, and N. Khan. Sharing it my way: Efficient M2M access in LTE/LTE-A Networks. IEEE Transactions on Vehicular Technology, 66(1):696-709, Jan 2017.

[62] V. B. Mišić, J. Mišić, X. Lin, and D. Nerandzic. Capillary machine-to-machine communications: The road ahead. In X.-Y. Li, S. Papavassiliou, and S. Ruehrup, editors, Ad-hoc, Mobile, and Wireless Networks, pages 413-423, Berlin, Heidelberg, 2012. Springer Berlin Heidelberg.

[63] S. Movassaghi, M. Abolhasan, J. Lipman, D. Smith, and A. Jamalipour. Wireless body area networks: A survey. IEEE Communications Surveys Tutorials, 16(3):16581686, Third 2014.

[64] I. C. Msadaa, P. Cataldi, and F. Filali. A comparative study between $802.11 \mathrm{p}$ and mobile WIMAX-based V2I communication networks. In 2010 Fourth International Con- 
ference on Next Generation Mobile Applications, Services and Technologies, pages 186-191, July 2010.

[65] J. A. Nazabal, F. Falcone, C. Fernndez-Valdivielso, and I. R. Matas. Development of a low mobility IEEE 802.15.4 compliant VANET system for urban environments. Sensors, 13(6):7065-7078, 2013.

[66] C. Otto, A. Milenković, C. Sanders, and E. Jovanov. System architecture of a wireless body area sensor network for ubiquitous health monitoring. J. Mob. Multimed., 1(4):307-326, Jan. 2005.

[67] S. Ozturk, J. Mišić, and V. B. Mišić. Reaching spatial or networking saturation in VANET. In Wireless Com Network, 2011.

[68] M. Polese, M. Centenaro, A. Zanella, and M. Zorzi. M2M massive access in LTE: RACH performance evaluation in a smart city scenario. In 2016 IEEE International Conference on Communications (ICC), pages 1-6, May 2016.

[69] S. Rashwand. Efficient Wireless Communication in Healthcare Systems; Design and Performance Evaluation. PhD thesis, The University of Manitoba, Winnipeg, Manitoba, Canada, August 2012.

[70] S. Rashwand and J. Mišić. Effects of access phases lengths on performance of IEEE 802.15.6 CSMA/CA. Computer Networks, 56(12):2832 - 2846, 2012.

[71] S. Rashwand, J. Mišić, and H. Khazaei. IEEE 802.15.6 under saturation: Some problems to be expected. Journal of Communications and Networks, 13(2):142-148, April 2011. 
[72] S. Rashwand, J. Mišić, and V. B. Mišić. Analysis of CSMA/CA mechanism of IEEE 802.15.6 under non-saturation regime. IEEE Transactions on Parallel and Distributed Systems, 27(5):1279-1288, May 2016.

[73] S. A. Salehi, M. A. Razzaque, I. Tomeo-Reyes, and N. Hussain. IEEE 802.15.6 standard in wireless body area networks from a healthcare point of view. In $201622 \mathrm{nd}$ Asia-Pacific Conference on Communications (APCC), pages 523-528, Aug 2016.

[74] S. Sesia, I. Toufik, and M. Baker. LTE, The UMTS Long Term Evolution: From Theory to Practice. Wiley Publishing, 2009.

[75] R. C. Shah, L. Nachman, and C.-y. Wan. On the performance of Bluetooth and IEEE 802.15.4 radios in a body area network. In Proceedings of the ICST 3rd International Conference on Body Area Networks, BodyNets '08, pages 25:1-25:9, ICST, Brussels, Belgium, Belgium, 2008. ICST (Institute for Computer Sciences, Social-Informatics and Telecommunications Engineering).

[76] IEEE Standard for Information Technology - Telecommunications and Information Exchange Between Systems - Local and Metropolitan Area Networks - Specific Requirements - Part 11: Wireless LAN Medium Access Control (MAC) and Physical Layer (PHY) Specifications, June 2007.

[77] 3GPP TS 37.868 V11.0. Study on RAN Improvements for Machine Type Communications, October 2011.

[78] N. F. Timmons and W. G. Scanlon. Analysis of the performance of IEEE 802.15.4 for medical sensor body area networking. In 2004 First Annual IEEE Communications 
Society Conference on Sensor and Ad Hoc Communications and Networks, 2004. IEEE SECON 2004., pages 16-24, Oct 2004.

[79] S. Ucar, S. Coleri Ergen, and O. Ozkasap. Multi-hop cluster based IEEE 802.11p and LTE hybrid architecture for VANET safety message dissemination. Vehicular Technology, IEEE Transactions on, $\mathrm{PP}(99): 1-1,2015$.

[80] P. Verma, R. Verma, A. Prakash, A. Agrawal, K. Naik, R. Tripathi, M. Alsabaan, T. Khalifa, T. Abdelkader, and A. Abogharaf. Machine-to-Machine (M2M) communications: A survey. Journal of Network and Computer Applications, 66:83 - 105, 2016.

[81] A. Vinel. 3GPP LTE versus IEEE 802.11p/WAVE: Which technology is able to support cooperative vehicular safety applications? Wireless Communications Letters, IEEE, 1(2):125-128, April 2012.

[82] J. Wan, D. Li, C. Zou, and K. Zhou. M2M communications for smart city: An eventbased architecture. In 2012 IEEE 12th International Conference on Computer and Information Technology, pages 895-900, Oct 2012.

[83] S. Wang, B. Yang, F. Gu, and X. Shi. A novel reliable broadcast protocol for VANET's safety applications. In 2016 6th International Conference on Electronics Information and Emergency Communication (ICEIEC), pages 282-286, June 2016.

[84] C. H. Wei, R. G. Cheng, and S. L. Tsao. Performance analysis of group paging for Machine-Type Communications in LTE networks. IEEE Transactions on Vehicular Technology, 62(7):3371-3382, Sept 2013. 
[85] Y. J. Wu, W. S. Wang, and W. S. Hwang. A modified backoff algorithm for safety message delivery in IEEE 802.11p/WAVE networks. In 2014 International Conference on Computer, Information and Telecommunication Systems (CITS), pages 1-5, July 2014.

[86] C. Xia, S. Xu, and K. S. Kwak. Resource allocation for device-to-device communication in LTE-A Network: A stackelberg game approach. In 2014 IEEE 80th Vehicular Technology Conference (VTC2014-Fall), pages 1-5, Sept 2014.

[87] S. Xu, J. Li, and X. Qin. Grouping based uplink resource allocation for massive M2M communications over LTE-A. In 2017 IEEE 86th Vehicular Technology Conference (VTC-Fall), pages 1-5, Sept 2017.

[88] G. Z. Yang. Body Sensor Networks. London: Springer-Verlag, 2006.

[89] Q. Yang, S. Xing, W. Xia, and L. Shen. Modelling and performance analysis of dynamic contention window scheme for periodic broadcast in vehicular ad hoc networks. IET Communications, 9(11):1347-1354, 2015.

[90] C. Zaouiat and A. Latif. Performances comparison of IEEE 802.15.6 and IEEE 802.15.4. IJACSA International Journal of Advanced Computer Science and Applications, 8(11), 2017.

[91] K. Zhou and N. Nikaein. Packet aggregation for machine type communications in LTE with random access channel. In 2013 IEEE Wireless Communications and Networking Conference (WCNC), pages 262-267, April 2013. 